\title{
Symmetries of supergravity backgrounds and supersymmetric field theory
}

\author{
Sergei M. Kuzenko and Emmanouil S.N. Raptakis \\ Department of Physics M013, The University of Western Australia, \\ 35 Stirling Highway, Perth W.A. 6009, Australia \\ E-mail: sergei.kuzenko@uwa.edu.au, \\ emmanouil.raptakis@research.uwa.edu.au
}

ABSTRACT: In four spacetime dimensions, all $\mathcal{N}=1$ supergravity-matter systems can be formulated in the so-called U(1) superspace proposed by Howe in 1981. This paper is devoted to the study of those geometric structures which characterise a background U(1) superspace and are important in the context of supersymmetric field theory in curved space. We introduce (conformal) Killing tensor superfields $\ell_{\left(\alpha_{1} \ldots \alpha_{m}\right)\left(\dot{\alpha}_{1} \ldots \dot{\alpha}_{n}\right)}$, with $m$ and $n$ non-negative integers, $m+n>0$, and elaborate on their significance in the following cases: (i) $m=n=1$; (ii) $m-1=n=0$; and (iii) $m=n>1$. The (conformal) Killing vector superfields $\ell_{\alpha \dot{\alpha}}$ generate the (conformal) isometries of curved superspace, which are symmetries of every (conformal) supersymmetric field theory. The (conformal) Killing spinor superfields $\ell_{\alpha}$ generate extended (conformal) supersymmetry transformations. The (conformal) Killing tensor superfields with $m=n>1$ prove to generate all higher symmetries of the (massless) massive Wess-Zumino operator.

KEYWORDS: Supergravity Models, Superspaces

ARXIV EPRINT: 1912.08552 


\section{Contents}

1 Introduction 1

2 The ABC of U(1) superspace $\quad 4$

2.1 The geometry of U(1) superspace 4

2.2 Super-Weyl transformations 6

2.3 From U(1) superspace to the Grimm-Wess-Zumino geometry 7

3 Conformal isometries of curved superspace $\quad 8$

3.1 Implications of the superconformal Killing equation 8

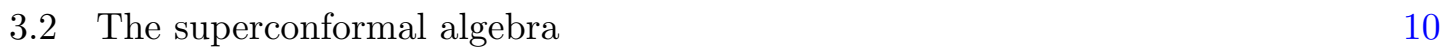

$\begin{array}{lll}3.3 & \text { Conformally related superspaces } & 12\end{array}$

$\begin{array}{lll}3.4 & \text { Superconformal field theory } & 12\end{array}$

$\begin{array}{ll}3.5 & \text { Superconformal sigma models } \\ \end{array}$

4 Conformal Killing tensor superfields $\quad 14$

$\begin{array}{lll}4.1 & \text { Definitions } & 15\end{array}$

$\begin{array}{lll}4.2 & \text { Conserved current supermultiplets } & 16\end{array}$

$\begin{array}{lll}4.3 & \text { Conformal Killing spinor superfields and hypermultiplet } & 17\end{array}$

4.4 Conformal Killing spinor superfields and nonlinear $\sigma$-models $\quad 19$

4.5 Symmetries of the massless Wess-Zumino operator 20

4.6 Supersymmetric even Schouten-Nijenhuis bracket 25

5 Isometries of curved superspace $\quad \mathbf{2 5}$

5.1 Off-shell supergravity and Killing vector superfields 26

$\begin{array}{ll}5.2 \text { Conformal compensators } & 27\end{array}$

5.3 Killing spinor superfields and massive hypermultiplet 28

5.4 Symmetries of the massive Wess-Zumino operator 28

6 Bosonic backgrounds $\quad 29$

$\begin{array}{lll}6.1 \text { Conformal isometries } & 30\end{array}$

6.2 Isometries of old minimal supergravity backgrounds 31

6.3 Isometries of new minimal supergravity backgrounds 32

6.4 Components of the (conformal) Killing tensor superfields 32

6.5 Components of conformal supercurrents 33

6.6 Maximally supersymmetric backgrounds 34

$\begin{array}{lll}7 & \text { Conclusion } & 35\end{array}$

$\begin{array}{ll}\text { A Chiral action } & 37\end{array}$ 
B Component reduction $\quad 38$

B.1 Wess-Zumino gauge 38

$\begin{array}{lll}\text { B.2 Component field strengths } & 39\end{array}$

C The Weyl multiplet gauge $\quad 41$

\section{Introduction}

In order to construct and study supersymmetric field theories in the presence of background supergravity fields, a formalism is required to determine (conformal) isometries of the corresponding curved superspace. ${ }^{1}$ Such a formalism was developed long ago [3] within the framework of the Grimm-Wess-Zumino (GWZ) geometry [4, 5], which underlies the WessZumino (WZ) formulation for old minimal supergravity [6] (see [7] for a review) discovered independently in [8-13]. The key outcomes of the analysis given in [3] may be summarised as follows:

- Rigid symmetries of every superconformal field theory on a curved superspace $\mathcal{M}^{4 \mid 4}$ are generated by conformal Killing supervector fields on $\mathcal{M}^{4 \mid 4}, \xi^{A}=\left(\xi^{a}, \xi^{\alpha}, \bar{\xi}_{\dot{\alpha}}\right)$, with $\bar{\xi}^{a}=\xi^{a}$. The defining property of $\xi^{A}$ is that the first-order operator $\xi^{A} \mathcal{D}_{A}$ maps the space of covariantly chiral scalars into itself,

$$
\overline{\mathcal{D}}_{\dot{\beta}} \phi=0 \quad \Longrightarrow \quad \overline{\mathcal{D}}_{\dot{\beta}}\left(\xi^{A} \mathcal{D}_{A} \phi\right)=0,
$$

where $\mathcal{D}_{A}=\left(\mathcal{D}_{a}, \mathcal{D}_{\alpha}, \overline{\mathcal{D}}^{\dot{\alpha}}\right)$ are the superspace covariant derivatives. These conditions imply that the spinor component $\xi^{\alpha}$ is determined in terms of the vector component $\xi^{a}$ as $\xi^{\alpha}=-\frac{\mathrm{i}}{8} \overline{\mathcal{D}}_{\dot{\beta}} \xi^{\alpha \dot{\beta}}$, and the latter obeys the superconformal Killing equation

$$
\mathcal{D}_{(\beta} \xi_{\alpha) \dot{\alpha}}=0 \quad \Longleftrightarrow \quad \overline{\mathcal{D}}_{(\dot{\beta}} \xi_{\alpha \dot{\alpha})}=0
$$

- Rigid symmetries of every supersymmetric field theory on $\mathcal{M}^{4 \mid 4}$ are associated with those conformal Killing supervector fields $\xi^{A}$ which preserve the volume of the chiral subspace of $\mathcal{M}^{4 \mid 4}$. This condition is equivalent to

$$
\mathcal{D}_{\alpha} \overline{\mathcal{D}}_{\dot{\alpha}} \xi^{\alpha \dot{\alpha}}=4 G_{\alpha \dot{\alpha}} \xi^{\alpha \dot{\alpha}} \quad \Longrightarrow \quad \mathcal{D}_{a} \xi^{a}=0,
$$

where $G_{\alpha \dot{\alpha}}$ is the superspace analogue of the Ricci tensor.

Every solution of the equations (1.2) and (1.3) is called a Killing supervector field.

If $\mathcal{M}^{4 \mid 4}$ is chosen to be Minkowski superspace, the general solution of the equation (1.2) corresponds to the ordinary superconformal transformations which span $\mathrm{SU}(2,2 \mid 1)$ [14-17].

\footnotetext{
${ }^{1}$ An important example of a curved superspace is the four-dimensional (4D) $\mathcal{N}=1$ anti-de Sitter (AdS) superspace $[1,2], \operatorname{AdS}^{4 \mid 4}$.
} 
In the case of supersymmetric curved backgrounds in old minimal supergravity, the equations (1.2) and (1.3) allow one to obtain all the results described in an influential work of Festuccia and Seiberg [18] and related publications (see e.g. [19, 20]) in the component setting, as was demonstrated in [21] (see also [22] for a review).

The approach presented in [3] is universal, for in principle it may be generalised to curved backgrounds associated with any supergravity theory formulated in superspace, see the discussion in [22]. In particular, it has been properly generalised to study supersymmetric backgrounds in 3D $\mathcal{N}=2$ supergravity [23], 4D $\mathcal{N}=2$ supergravity [24], 5D $\mathcal{N}=1$ supergravity [25] and $6 \mathrm{D} \mathcal{N}=(1,0)$ supergravity [26]. It should also be mentioned that this approach has been used to construct general rigid supersymmetric field theories in 5D $\mathcal{N}=1[27], 4 \mathrm{D} \mathcal{N}=2[28-31]$ and 3D $(p, q)[32-34]$ anti-de Sitter superspaces.

The present paper is aimed, in part, at extending the analysis given in section 6.4 of [3] to the so-called U(1) superspace geometry proposed by Howe in $1981[35,36]$ and soon after reviewed and further developed in [37]. ${ }^{2}$ It is called ' $U(1)$ superspace' since its structure group $\mathrm{SL}(2, \mathbb{C}) \times \mathrm{U}(1)_{R}$ contains the $R$-symmetry factor $\mathrm{U}(1)_{R}$ that is absent in the case of the GWZ geometry $[4,5]$. The $\mathrm{U}(1)$ superspace is a powerful setting to formulate $\mathcal{N}=1$ supergravity-matter systems for two reasons. Firstly, it allows us to describe conformal supergravity by including the super-Weyl transformations in the supergravity gauge group. Secondly, every off-shell formulation for $\mathcal{N}=1$ supergravity can be realised as a superWeyl invariant coupling of conformal supergravity to a compensating supermultiplet $\Xi$. In fact, similar properties also hold in the case of the GWZ geometry. One may then ask a natural question: what is the point of introducing $\mathrm{U}(1)$ superspace if the GWZ geometry allows one to achieve the same goals? There are at least three answers to this question. Firstly, the GWZ geometry is a gauge-fixed version of U(1) superspace in the sense that the former is obtained from the latter by partially fixing the super-Weyl gauge symmetry. Secondly, since the super-Weyl and local $\mathrm{U}(1)_{R}$ transformations are described by unconstrained real parameters in $\mathrm{U}(1)$ superspace, these local symmetries may be used to gauge away any compensating scalar supermultiplet $\Xi$ by imposing the condition $\Xi=1$. In the case of the GWZ geometry, such a gauge fixing is possible only in the case of old minimal supergravity. Thirdly, U(1) superspace is more useful for describing the new minimal formulation of $\mathcal{N}=1$ supergravity [38, 39]. ${ }^{3}$

Along with the (conformal) Killing vector superfields $\xi_{\alpha \dot{\alpha}}$, which generate the (conformal) isometries of a curved superspace $\mathcal{M}^{4 \mid 4}$, in this paper (sections 4 and 5) we will analyse the structure of (conformal) Killing tensor superfields $\ell_{\alpha(m) \dot{\alpha}(n)}=\ell_{\left(\alpha_{1} \ldots \alpha_{m}\right)\left(\dot{\alpha}_{1} \ldots \dot{\alpha}_{n}\right)}$, with $m$ and $n$ non-negative integers, $m+n>0$. Some of the motivations to study these supersymmetric extensions of the (conformal) Killing tensor fields are similar to those that

\footnotetext{
${ }^{2}$ One of the most important original developments presented in [37] is the complete solution of the torsion constraints, which characterise the U(1) superspace geometry, in terms of unconstrained superfield prepotentials.

${ }^{3}$ There exists an alternative formulation for conformal supergravity, the so-called conformal superspace approach [40], which is more general than $U(1)$ superspace in the sense that the latter is obtained from the former by partially fixing the gauge freedom. When studying the symmetries of supergravity backgrounds, however, $\mathrm{U}(1)$ superspace is more economical for applications to deal with.
} 
have been pursued in the non-supersymmetric case, which are: (i) higher-order integrals of motion, see e.g. [41]; (ii) new conserved currents from old ones, see e.g. [42]; and (iii) higher symmetries of relativistic wave equations, see e.g. [43-48]. There are also conceptually new motivations. In particular, if a curved superspace $\mathcal{M}^{4 \mid 4}$ possesses a (conformal) Killing spinor superfield $\ell_{\alpha}$, extended supersymmetric field theories may be constructed, including superconformal nonlinear $\sigma$-models on hyperkähler cones, see section 4.4.

The concept of a Killing tensor superfield $\ell_{\alpha(n) \dot{\alpha}(n)}=\bar{\ell}_{\alpha(n) \dot{\alpha}(n)}$ was introduced in 1997 [49] in the framework of $\mathcal{N}=1$ AdS supersymmetry. There are two types of constraints obeyed by $\ell_{\alpha(n) \dot{\alpha}(n)}$, which are:

$$
\begin{array}{rlrl}
\mathcal{D}_{\left(\alpha_{1}\right.} \ell_{\left.\alpha_{2} \ldots \alpha_{n+1}\right) \dot{\alpha}(n)} & =0 & \Longleftrightarrow & \overline{\mathcal{D}}_{\left(\dot{\alpha}_{1} \ell_{\left.\alpha(n) \dot{\alpha}_{2} \ldots \dot{\alpha}_{n+1}\right)}\right.}=0, \\
\mathcal{D}^{\beta} \overline{\mathcal{D}}^{\dot{\beta}} \ell_{\beta \alpha_{1} \ldots \alpha_{n-1} \dot{\beta} \dot{\alpha}_{1} \ldots \dot{\alpha}_{n-1}}=0 & \Longleftrightarrow & \overline{\mathcal{D}}^{\dot{\beta}} \mathcal{D}^{\beta} \ell_{\beta \alpha_{1} \ldots \alpha_{n-1} \dot{\beta} \dot{\alpha}_{1} \ldots \dot{\alpha}_{n-1}}=0 .
\end{array}
$$

These differential constraints have a natural origin in the context of the two dually equivalent gauge models for the massless superspin- $\left(n+\frac{1}{2}\right)$ multiplet in $\operatorname{AdS}^{4 \mid 4}$ which were proposed in [50]. The dynamical variables of these models consist of a gauge superfield and a compensating supermultiplet. In both models the gauge superfield is the same, that is a real unconstrained superconformal prepotential $H_{\alpha(n) \dot{\alpha}(n)}$, while the compensators are different. In one model the compensator is a transverse linear superfield $\Gamma_{\alpha(n-2) \dot{\alpha}(n-2)}$, and in the other is it a longitudinal linear superfield $G_{\alpha(n-2) \dot{\alpha}(n-2)} \cdot{ }^{4}$ The corresponding constraints are

$$
\begin{aligned}
\overline{\mathcal{D}}^{\dot{\beta}} \Gamma_{\alpha(n-2) \dot{\beta} \dot{\alpha}(n-3)} & =0, \\
\overline{\mathcal{D}}_{\left(\dot{\alpha}_{1}\right.} G_{\left.\alpha(n-2) \dot{\alpha}_{2} \ldots \dot{\alpha}_{n-1}\right)} & =0 .
\end{aligned}
$$

Equation (1.4a) means that the gauge variation of $H_{\alpha(n) \dot{\alpha}(n)}$ is equal to zero if the gauge parameter is chosen to be $\ell_{\alpha(n) \dot{\alpha}(n-1)}$. In addition, requiring the gauge variation of the compensator (either the transverse or the longitudinal one) to vanish leads to the equation (1.4b). It was shown in [49] that the space of Killing tensor superfields $\ell_{\alpha(n) \dot{\alpha}(n)}$ can be endowed with the structure of a superalgebra, which is one of the higher-spin superalgebras constructed by Fradkin and Vasiliev [52-54] (see also $[55,56]$ ), with respect to the bracket (4.63) restricted to $\mathrm{AdS}^{4 \mid 4}$. A conformal Killing tensor superfield $\ell_{\alpha(n) \dot{\alpha}(n)}$ in $\mathrm{AdS}^{4 \mid 4}$ is obtained by removing the condition (1.4b) which is not compatible with the superconformal symmetry (this aspect was not discussed explicitly in [49]).

In 2016, Howe and Lindström [57] generalised the notion of a conformal Killing tensor to superspace in diverse dimensions. ${ }^{5}$ In the case of $4 \mathrm{D} \mathcal{N}=1$ AdS supersymmetry, their definition is equivalent to imposing the condition (1.4a). Our definition of conformal Killing tensor superfields in curved superspace differs from the one given in [57], however they prove to be equivalent.

This paper is organised as follows. Section 2 is devoted to a brief review of U(1) superspace. The conformal isometries of a supergravity background are studied in section 3. We also describe the action principle for superconformal field theories in a curved

\footnotetext{
${ }^{4}$ The terminology follows [50, 51].

${ }^{5}$ See also $[58,59]$ for related work in which they discussed the relation of superconformal Killing tensors to higher dimensional supercurrents, superconformal Killing-Yano tensors, super-Laplacians etc.
} 
superspace and give an example of such dynamical systems - a superconformal nonlinear $\sigma$-model. Section 4 is devoted to a systematic study of conformal Killing tensor superfields $\ell_{\alpha(m) \dot{\alpha}(n)}$ in curved superspace. We demonstrate the significance of different types of conformal Killing tensor superfields for various superconformal field theories in curved superspace. The isometries of a supergravity background are studied in section 5 . We also introduce Killing spinor $\ell_{\alpha}$ and tensor $\ell_{\alpha(n) \dot{\alpha}(n)}$ superfields and demonstrate their significance for several supersymmetric field theories in curved superspace. The symmetries of bosonic supergravity backgrounds are studied in section 6 . Concluding comments are given in section 7. The main body of the paper is accompanied by several technical appendices. Appendix A is devoted to the closed super 4-form which describes the chiral action principle. Appendix B concerns various aspects of the component reduction. The Weyl multiplet gauge is introduced in appendix $\mathrm{C}$.

\section{The ABC of $\mathrm{U}(1)$ superspace}

In this section we review the structure of $\mathrm{U}(1)$ superspace [35-37]. Our presentation is analogous to [60].

\subsection{The geometry of $U(1)$ superspace}

We consider a curved $\mathcal{N}=1$ superspace $\mathcal{M}^{4 \mid 4}$ parametrised by local coordinates $z^{M}=$ $\left(x^{m}, \theta^{\mu}, \bar{\theta}_{\dot{\mu}}\right)$. Its structure group is chosen to be $\mathrm{SL}(2, \mathbb{C}) \times \mathrm{U}(1)_{R}$ and so the covariant derivatives $\mathcal{D}_{A}=\left(\mathcal{D}_{a}, \mathcal{D}_{\alpha}, \overline{\mathcal{D}}^{\dot{\alpha}}\right)$ have the form

$$
\mathcal{D}_{A}=E_{A}+\Omega_{A}+\mathrm{i} \Phi_{A} \mathbb{A} .
$$

Here $E_{A}$ denotes the frame field, $E_{A}=E_{A}{ }^{M} \partial_{M}$, with $E_{A}{ }^{M}$ being the inverse vielbein. The Lorentz connection $\Omega_{A}$ can be written in two different forms,

$$
\Omega_{A}=\frac{1}{2} \Omega_{A}^{b c} M_{b c}=\Omega_{A}^{\beta \gamma} M_{\beta \gamma}+\bar{\Omega}_{A} \dot{\beta} \dot{\gamma} \bar{M}_{\dot{\beta} \dot{\gamma}},
$$

depending on whether the Lorentz generators with vector $\left(M_{b c}=-M_{c b}\right)$ or spinor $\left(M_{\beta \gamma}=\right.$ $M_{\gamma \beta}$ and $\left.\bar{M}_{\dot{\beta} \dot{\gamma}}=\bar{M}_{\dot{\gamma} \dot{\beta}}\right)$ indices are used. The Lorentz generators act on vectors and Weyl spinors as follows:

$$
M_{a b} V_{c}=2 \eta_{c[a} V_{b]}, \quad M_{\alpha \beta} \psi_{\gamma}=\varepsilon_{\gamma(\alpha} \psi_{\beta)}, \quad \bar{M}_{\dot{\alpha} \dot{\beta}} \bar{\psi}_{\dot{\gamma}}=\varepsilon_{\dot{\gamma}(\dot{\alpha}} \bar{\psi}_{\dot{\beta})}
$$

The last term in $(2.1)$ is the $\mathrm{U}(1)_{R}$ connection, with the $R$-symmetry generator $\mathbb{A}$ being normalised by

$$
\left[\mathbb{A}, \mathcal{D}_{\alpha}\right]=-\mathcal{D}_{\alpha}, \quad\left[\mathbb{A}, \overline{\mathcal{D}}_{\dot{\alpha}}\right]=+\overline{\mathcal{D}}_{\dot{\alpha}}
$$

The supergravity gauge freedom includes local $\mathcal{K}$-transformations of the form

$$
\delta_{\mathcal{K}} \mathcal{D}_{A}=\left[\mathcal{K}, \mathcal{D}_{A}\right], \quad \mathcal{K}=\xi^{B} \mathcal{D}_{B}+K^{\beta \gamma} M_{\beta \gamma}+\bar{K}^{\dot{\beta} \dot{\gamma}} \bar{M}_{\dot{\beta} \dot{\gamma}}+\mathrm{i} \rho \mathbb{A}
$$


Here the gauge parameter $\mathcal{K}$ incorporates several parameters describing the general coordinate $\left(\xi^{B}\right)$, local Lorentz $\left(K^{\beta \gamma}\right.$ and $\left.\bar{K}^{\dot{\beta} \dot{\gamma}}\right)$ and local chiral $(\rho)$ transformations. Given a tensor superfield $U$ (with suppressed indices), its $\mathcal{K}$-transformation law is

$$
\delta_{\mathcal{K}} U=\mathcal{K} U
$$

The covariant derivatives obey graded commutation relations

$$
\left[\mathcal{D}_{A}, \mathcal{D}_{B}\right\}=\mathcal{T}_{A B}{ }^{C} \mathcal{D}_{C}+\mathcal{R}_{A B}{ }^{\gamma \delta} M_{\gamma \delta}+\overline{\mathcal{R}}_{A B} \dot{\gamma} \dot{\delta} \bar{M}_{\dot{\gamma} \dot{\delta}}+\mathrm{i} \mathcal{F}_{A B} \mathbb{A}
$$

where $\mathcal{T}_{A B}^{C}$ is the torsion, $\mathcal{R}_{A B}{ }^{\gamma \delta}$ and its conjugate $\mathcal{R}_{A B}{ }^{\dot{\gamma} \dot{\delta}}$ constitute the Lorentz curvature, and $\mathcal{F}_{A B}$ is the $\mathrm{U}(1)_{R}$ field strength. To describe conformal supergravity, the covariant derivatives have to obey certain constraints $[35,36]$. Their solution is given by the relations

$$
\begin{aligned}
\left\{\mathcal{D}_{\alpha}, \mathcal{D}_{\beta}\right\}= & -4 \bar{R} M_{\alpha \beta}, \quad\left\{\overline{\mathcal{D}}_{\dot{\alpha}}, \overline{\mathcal{D}}_{\dot{\beta}}\right\}=4 R \bar{M}_{\dot{\alpha} \dot{\beta}}, \\
\left\{\mathcal{D}_{\alpha}, \overline{\mathcal{D}}_{\dot{\alpha}}\right\}= & -2 \mathrm{i} \mathcal{D}_{\alpha \dot{\alpha}}, \\
{\left[\mathcal{D}_{\alpha}, \mathcal{D}_{\beta \dot{\beta}}\right]=} & \mathrm{i} \varepsilon_{\alpha \beta}\left(\bar{R} \overline{\mathcal{D}}_{\dot{\beta}}+G_{\dot{\beta}}^{\gamma} \mathcal{D}_{\gamma}-\left(\mathcal{D}^{\gamma} G_{\dot{\beta}}^{\delta}\right) M_{\gamma \delta}+2 \bar{W}_{\dot{\beta}}^{\dot{\gamma} \dot{\delta}} \bar{M}_{\dot{\gamma} \dot{\delta}}\right) \\
& +\mathrm{i}\left(\overline{\mathcal{D}}_{\dot{\beta}} \bar{R}\right) M_{\alpha \beta}-\frac{\mathrm{i}}{3} \varepsilon_{\alpha \beta} \bar{X}^{\dot{\gamma}} \bar{M}_{\dot{\gamma} \dot{\beta}}+\frac{\mathrm{i}}{2} \varepsilon_{\alpha \beta} \bar{X}_{\dot{\beta}} \mathbb{A}, \\
{\left[\overline{\mathcal{D}}_{\dot{\alpha}}, \mathcal{D}_{\beta \dot{\beta}}\right]=} & -\mathrm{i} \varepsilon_{\dot{\alpha} \dot{\beta}}\left(R \mathcal{D}_{\beta}+G_{\beta}^{\dot{\gamma}} \overline{\mathcal{D}}_{\dot{\gamma}}-\left(\overline{\mathcal{D}}^{\dot{\gamma}} G_{\beta} \dot{\delta}\right) \bar{M}_{\dot{\gamma} \dot{\delta}}+2 W_{\beta}^{\gamma \delta} M_{\gamma \delta}\right) \\
& -\mathrm{i}\left(\mathcal{D}_{\beta} R\right) \bar{M}_{\dot{\alpha} \dot{\beta}}+\frac{\mathrm{i}}{3} \varepsilon_{\dot{\alpha} \dot{\beta}} X^{\gamma} M_{\gamma \beta}+\frac{\mathrm{i}}{2} \varepsilon_{\dot{\alpha} \dot{\beta}} X_{\beta} \mathbb{A},
\end{aligned}
$$

which lead to

$$
\begin{aligned}
{\left[\mathcal{D}_{\alpha \dot{\alpha}}, \mathcal{D}_{\beta \dot{\beta}}\right]=} & \varepsilon_{\alpha \beta} \bar{\psi}_{\dot{\alpha} \dot{\beta}}+\varepsilon_{\dot{\alpha} \dot{\beta}} \psi_{\alpha \beta}, \\
\psi_{\alpha \beta}= & -\mathrm{i} G_{(\alpha}{ }^{\dot{\gamma}} \mathcal{D}_{\beta) \dot{\gamma}}+\frac{1}{2} \mathcal{D}_{(\alpha} R \mathcal{D}_{\beta)}+\frac{1}{2} \mathcal{D}_{(\alpha} G_{\beta)} \dot{\gamma} \overline{\mathcal{D}}_{\dot{\gamma}}+W_{\alpha \beta}{ }^{\gamma} \mathcal{D}_{\gamma} \\
& +\frac{1}{6} X_{(\alpha} \mathcal{D}_{\beta)}+\frac{1}{4}\left(\mathcal{D}^{2}-8 R\right) \bar{R} M_{\alpha \beta}+\mathcal{D}_{(\alpha} W_{\beta)}{ }^{\gamma \delta} M_{\gamma \delta} \\
& -\frac{1}{6} \mathcal{D}_{(\alpha} X^{\gamma} M_{\beta) \gamma}-\frac{1}{2} \mathcal{D}_{(\alpha} \overline{\mathcal{D}}^{\dot{\gamma}} G_{\beta)} \dot{\delta} \bar{M}_{\dot{\gamma} \dot{\delta}}-\frac{1}{4} \mathcal{D}_{(\alpha} X_{\beta)} \mathbb{A} \\
\bar{\psi}_{\dot{\alpha} \dot{\beta}}= & \mathrm{i} G^{\gamma}{ }_{(\dot{\alpha}} \mathcal{D}_{\gamma \dot{\beta})}-\frac{1}{2} \overline{\mathcal{D}}_{(\dot{\alpha}} \bar{R} \overline{\mathcal{D}}_{\dot{\beta})}-\frac{1}{2} \overline{\mathcal{D}}_{(\dot{\alpha}} G_{\dot{\beta})}^{\gamma} \mathcal{D}_{\gamma}-\bar{W}_{\dot{\alpha} \dot{\beta}} \dot{\gamma} \overline{\mathcal{D}}_{\dot{\gamma}} \\
& -\frac{1}{6} \bar{X}_{(\dot{\alpha}} \overline{\mathcal{D}}_{\dot{\beta})}+\frac{1}{4}\left(\overline{\mathcal{D}}^{2}-8 \bar{R}\right) R \bar{M}_{\dot{\alpha} \dot{\beta}}-\overline{\mathcal{D}}_{(\dot{\alpha}} \bar{W}_{\dot{\beta})} \dot{\gamma} \dot{\delta} \bar{M}_{\dot{\gamma} \dot{\delta}} \\
& +\frac{1}{6} \overline{\mathcal{D}}_{(\dot{\alpha}} \bar{X}^{\dot{\gamma}} \bar{M}_{\dot{\beta}) \dot{\gamma}}+\frac{1}{2} \overline{\mathcal{D}}_{(\dot{\alpha}} \mathcal{D}^{\gamma} G_{\dot{\beta})}^{\delta} M_{\gamma \delta}-\frac{1}{4} \overline{\mathcal{D}}_{(\dot{\alpha}} \bar{X}_{\dot{\beta})} \mathbb{A}
\end{aligned}
$$

The torsion and curvature tensors are expressed in terms of the real vector $G_{a}$ and the complex superfields $R, X_{\alpha}$ and $W_{\alpha \beta \gamma}=W_{(\alpha \beta \gamma)}$, which have the $\mathrm{U}(1)_{R}$ charges

$$
\mathbb{A} R=2 R, \quad \mathbb{A} X_{\alpha}=X_{\alpha}, \quad \mathbb{A} W_{\alpha \beta \gamma}=W_{\alpha \beta \gamma} .
$$

and are covariantly chiral,

$$
\overline{\mathcal{D}}_{\dot{\alpha}} R=0, \quad \overline{\mathcal{D}}_{\dot{\alpha}} X_{\alpha}=0, \quad \overline{\mathcal{D}}_{\dot{\alpha}} W_{\alpha \beta \gamma}=0 .
$$


These superfields obey the following Bianchi identities:

$$
\begin{aligned}
X_{\alpha} & =\mathcal{D}_{\alpha} R-\overline{\mathcal{D}}^{\dot{\alpha}} G_{\alpha \dot{\alpha}}, \\
\mathcal{D}^{\alpha} X_{\alpha} & =\overline{\mathcal{D}}_{\dot{\alpha}} \bar{X}^{\dot{\alpha}}, \\
\mathcal{D}^{\gamma} W_{\alpha \beta \gamma} & =\mathrm{i} \mathcal{D}_{(\alpha}{ }^{\dot{\gamma}} G_{\beta) \dot{\gamma}}-\frac{1}{3} \mathcal{D}_{(\alpha} X_{\beta)} .
\end{aligned}
$$

Equation (2.11b) means that $X_{\alpha}$ is the chiral field strength of an Abelian vector multiplet.

In what follows we will use the notation $\left(\mathcal{M}^{4 \mid 4}, \mathcal{D}\right)$ for the superspace $\mathcal{M}^{4 \mid 4}$ endowed with the geometry described.

\subsection{Super-Weyl transformations}

In order for the above superspace geometry to describe conformal supergravity, the supergravity gauge group should include super-Weyl transformations, with the corresponding parameter $\Sigma$ being a real unconstrained scalar superfield. The defining property of these local rescalings is that they preserve the structure of the algebra of covariant derivatives. In the infinitesimal case, the super-Weyl transformation is

$$
\begin{aligned}
\delta_{\Sigma} \mathcal{D}_{\alpha}= & \frac{1}{2} \Sigma \mathcal{D}_{\alpha}+2 \mathcal{D}^{\beta} \Sigma M_{\beta \alpha}+\frac{3}{2} \mathcal{D}_{\alpha} \Sigma \mathbb{A} \\
\delta_{\Sigma} \overline{\mathcal{D}}_{\dot{\alpha}}= & \frac{1}{2} \Sigma \overline{\mathcal{D}}_{\dot{\alpha}}+2 \overline{\mathcal{D}}^{\dot{\beta}} \Sigma \bar{M}_{\dot{\beta} \dot{\alpha}}-\frac{3}{2} \overline{\mathcal{D}}_{\dot{\alpha}} \Sigma \mathbb{A} \\
\delta_{\Sigma} \mathcal{D}_{\alpha \dot{\alpha}}= & \Sigma \mathcal{D}_{\alpha \dot{\alpha}}+\mathrm{i} \mathcal{D}_{\alpha} \Sigma \overline{\mathcal{D}}_{\dot{\alpha}}+\mathrm{i} \overline{\mathcal{D}}_{\dot{\alpha}} \Sigma \mathcal{D}_{\alpha}+\mathrm{i} \overline{\mathcal{D}}_{\dot{\alpha}} \mathcal{D}^{\beta} \Sigma M_{\beta \alpha} \\
& +\mathrm{i} \mathcal{D}_{\alpha} \overline{\mathcal{D}}^{\dot{\beta}} \Sigma \bar{M}_{\dot{\beta} \dot{\alpha}}-\frac{3}{4} \mathrm{i}\left[\mathcal{D}_{\alpha}, \overline{\mathcal{D}}_{\dot{\alpha}}\right] \Sigma \mathbb{A}
\end{aligned}
$$

and the corresponding variations of the torsion and curvature superfields are

$$
\begin{aligned}
\delta_{\Sigma} R & =\Sigma R+\frac{1}{2} \overline{\mathcal{D}}^{2} \Sigma \\
\delta_{\Sigma} G_{\alpha \dot{\alpha}} & =\Sigma G_{\alpha \dot{\alpha}}+\left[\mathcal{D}_{\alpha}, \overline{\mathcal{D}}_{\dot{\alpha}}\right] \Sigma, \\
\delta_{\Sigma} W_{\alpha \beta \gamma} & =\frac{3}{2} \Sigma W_{\alpha \beta \gamma}, \\
\delta_{\Sigma} X_{\alpha} & =\frac{3}{2} \Sigma X_{\alpha}-\frac{3}{2}\left(\overline{\mathcal{D}}^{2}-4 R\right) \mathcal{D}_{\alpha} \Sigma .
\end{aligned}
$$

In appendix $\mathrm{C}$ we demonstrate that the gauge transformations (2.5) and (2.12) allow us to choose a Wess-Zumino gauge in which the remaining fields constitute the Weyl multiplet of conformal supergravity.

Consider a tensor superfield $U$ of $\mathrm{U}(1)_{R}$ charge $q_{U}$,

$$
\mathbb{A} U=q_{U} U
$$

It is called primary if its super-Weyl transformation has the form

$$
\delta_{\Sigma} U=\Delta_{U} \Sigma U
$$


for some parameter $\Delta_{U}$ called the dimension of $U$. Given a primary superfield $\Psi_{\alpha_{1} \ldots \alpha_{n}}=$ $\Psi_{\left(\alpha_{1} \ldots \alpha_{n}\right)}$, which is covariantly chiral, $\overline{\mathcal{D}}_{\dot{\beta}} \Psi_{\alpha_{1} \ldots \alpha_{n}}=0$, its dimensions and $\mathrm{U}(1)_{R}$ charge are related to each other by

$$
q_{\Psi}=\frac{2}{3} \Delta_{\Psi}
$$

For completeness, we also provide the finite super-Weyl transformation. It is

$$
\begin{aligned}
\mathcal{D}_{\alpha}^{\prime}= & \mathrm{e}^{\frac{1}{2} \Sigma}\left(\mathcal{D}_{\alpha}+2 \mathcal{D}^{\beta} \Sigma M_{\beta \alpha}+\frac{3}{2} \mathcal{D}_{\alpha} \Sigma \mathbb{A}\right) \\
\overline{\mathcal{D}}_{\dot{\alpha}}^{\prime}= & \mathrm{e}^{\frac{1}{2} \Sigma}\left(\overline{\mathcal{D}}_{\dot{\alpha}}+2 \overline{\mathcal{D}}^{\dot{\beta}} \Sigma \bar{M}_{\dot{\beta} \dot{\alpha}}-\frac{3}{2} \overline{\mathcal{D}}_{\dot{\alpha}} \Sigma \mathbb{A}\right) \\
\mathcal{D}_{\alpha \dot{\alpha}}^{\prime}= & \mathrm{e}^{\Sigma}\left(\mathcal{D}_{\alpha \dot{\alpha}}+\mathrm{i} \mathcal{D}_{\alpha} \Sigma \overline{\mathcal{D}}_{\dot{\alpha}}+\mathrm{i} \overline{\mathcal{D}}_{\dot{\alpha}} \Sigma \mathcal{D}_{\alpha}+\mathrm{i}\left(\overline{\mathcal{D}}_{\dot{\alpha}} \mathcal{D}^{\beta} \Sigma+2 \overline{\mathcal{D}}_{\dot{\alpha}} \Sigma \mathcal{D}^{\beta} \Sigma\right) M_{\beta \alpha}\right. \\
& +\mathrm{i}\left(\mathcal{D}_{\alpha} \overline{\mathcal{D}}^{\dot{\beta}} \Sigma+2 \mathcal{D}_{\alpha} \Sigma \overline{\mathcal{D}}^{\dot{\beta}} \Sigma\right) \bar{M}_{\dot{\beta} \dot{\alpha}} \\
& \left.-3 \mathrm{i}\left(\frac{1}{4}\left[\mathcal{D}_{\alpha}, \overline{\mathcal{D}}_{\dot{\alpha}}\right] \Sigma+\mathcal{D}_{\alpha} \Sigma \overline{\mathcal{D}}_{\dot{\alpha}} \Sigma\right) \mathbb{A}\right) .
\end{aligned}
$$

The corresponding transformation laws for the torsion and curvature superfields are

$$
\begin{aligned}
R^{\prime} & =\mathrm{e}^{\Sigma}\left(R+\frac{1}{2} \overline{\mathcal{D}}^{2} \Sigma-(\overline{\mathcal{D}} \Sigma)^{2}\right), \\
G_{\alpha \dot{\alpha}}^{\prime} & =\mathrm{e}^{\Sigma}\left(G_{\alpha \dot{\alpha}}+\left[\mathcal{D}_{\alpha}, \overline{\mathcal{D}}_{\dot{\alpha}}\right] \Sigma+2 \mathcal{D}_{\alpha} \Sigma \overline{\mathcal{D}}_{\dot{\alpha}} \Sigma\right), \\
W_{\alpha \beta \gamma}^{\prime} & =\mathrm{e}^{\frac{3}{2} \Sigma} W_{\alpha \beta \gamma}, \\
X_{\alpha}^{\prime} & =\mathrm{e}^{\frac{3}{2} \Sigma}\left(X_{\alpha}-\frac{3}{2}\left(\overline{\mathcal{D}}^{2}-4 R\right) \mathcal{D}_{\alpha} \Sigma\right) .
\end{aligned}
$$

The super-Weyl tensor $W_{\alpha \beta \gamma}$ and its conjugate $\bar{W}_{\dot{\alpha} \dot{\beta} \dot{\gamma}}$ are the only torsion superfields which transform homogeneously under the super-Weyl group.

\subsection{From U(1) superspace to the Grimm-Wess-Zumino geometry}

As pointed out above, the covariantly chiral spinor $X_{\alpha}$ is the field strength of an Abelian vector multiplet. It follows from (2.18d) that the super-Weyl gauge freedom allows us to choose the gauge

$$
X_{\alpha}=0
$$

In this gauge the $\mathrm{U}(1)_{R}$ curvature vanishes, in accordance with (2.8), and therefore the $\mathrm{U}(1)_{R}$ connection may be gauged away,

$$
\Phi_{A}=0 .
$$

As a result, the algebra of covariant derivatives reduces (2.8) reduces to that describing the GWZ geometry $[4,5]$. 
Equation (2.18d) tells us that imposing the condition $X_{\alpha}=0$ does not fix completely the super-Weyl freedom. The residual transformations are generated by parameters of the form

$$
\Sigma=\frac{1}{2}(\sigma+\bar{\sigma}), \quad \overline{\mathcal{D}}_{\dot{\alpha}} \sigma=0
$$

However, in order to preserve the $\mathrm{U}(1)_{R}$ gauge $\Phi_{A}=0$, every residual super-Weyl transformation (2.21) must be accompanied by a compensating $\mathrm{U}(1)_{R}$ transformation with

$$
\rho=\frac{3}{4} \mathrm{i}(\bar{\sigma}-\sigma)
$$

This leads to the transformation $[61,62]$

$$
\begin{aligned}
\delta_{\sigma} \mathcal{D}_{\alpha}= & \left(\bar{\sigma}-\frac{1}{2} \sigma\right) \mathcal{D}_{\alpha}+\left(\mathcal{D}^{\beta} \sigma\right) M_{\alpha \beta}, \\
\delta_{\sigma} \overline{\mathcal{D}}_{\dot{\alpha}}= & \left(\sigma-\frac{1}{2} \bar{\sigma}\right) \overline{\mathcal{D}}_{\dot{\alpha}}+\left(\overline{\mathcal{D}}^{\dot{\beta}} \bar{\sigma}\right) \bar{M}_{\dot{\alpha} \dot{\beta}}, \\
\delta_{\sigma} \mathcal{D}_{\alpha \dot{\alpha}}= & \frac{1}{2}(\sigma+\bar{\sigma}) \mathcal{D}_{\alpha \dot{\alpha}}+\frac{\mathrm{i}}{2}\left(\overline{\mathcal{D}}_{\dot{\alpha}} \bar{\sigma}\right) \mathcal{D}_{\alpha}+\frac{\mathrm{i}}{2}\left(\mathcal{D}_{\alpha} \sigma\right) \overline{\mathcal{D}}_{\dot{\alpha}} \\
& +\left(\mathcal{D}^{\beta}{ }_{\dot{\alpha}} \sigma\right) M_{\alpha \beta}+\left(\mathcal{D}_{\alpha} \dot{\beta} \bar{\sigma}\right) \bar{M}_{\dot{\alpha} \dot{\beta}}
\end{aligned}
$$

The torsion tensors transform as follows:

$$
\begin{aligned}
\delta_{\sigma} R & =2 \sigma R+\frac{1}{4}\left(\overline{\mathcal{D}}^{2}-4 R\right) \bar{\sigma} \\
\delta_{\sigma} G_{\alpha \dot{\alpha}} & =\frac{1}{2}(\sigma+\bar{\sigma}) G_{\alpha \dot{\alpha}}+\mathrm{i} \mathcal{D}_{\alpha \dot{\alpha}}(\sigma-\bar{\sigma}), \\
\delta_{\sigma} W_{\alpha \beta \gamma} & =\frac{3}{2} \sigma W_{\alpha \beta \gamma} .
\end{aligned}
$$

\section{Conformal isometries of curved superspace}

Let $\left(\mathcal{M}^{4 \mid 4}, \mathcal{D}\right)$ be a background superspace. A real supervector field $\xi=\xi^{B} E_{B}$ is called conformal Killing if

$$
\left(\delta_{\mathcal{K}}+\delta_{\Sigma}\right) \mathcal{D}_{A}=0
$$

for some Lorentz $\left(K^{\beta \gamma}\right)$, chiral $(\rho)$ and super-Weyl $(\Sigma)$ parameters. Every solution to (3.1) defines a superconformal transformation of the superspace $\left(\mathcal{M}^{4 \mid 4}, \mathcal{D}\right)$.

\subsection{Implications of the superconformal Killing equation}

Equation (3.1) contains nontrivial information. Choosing $A=\alpha$ in (3.1) and making use of the definition (2.5) and (2.12) in conjunction with the graded commutation relations (2.8), 
we derive

$$
\begin{aligned}
\left(\delta_{\mathcal{K}}+\delta_{\Sigma}\right) \mathcal{D}_{\alpha}= & \left(K_{\alpha}{ }^{\beta}-\mathcal{D}_{\alpha} \xi^{\beta}-\frac{\mathrm{i}}{2} \xi_{\alpha \dot{\beta}} G^{\beta \dot{\beta}}-\mathrm{i} \delta_{\alpha}{ }^{\beta} \rho+\frac{1}{2} \delta_{\alpha}{ }^{\beta} \Sigma\right) \mathcal{D}_{\beta} \\
& +\left(\mathcal{D}_{\alpha} \bar{\xi}^{\dot{\beta}}+\frac{\mathrm{i}}{2} \xi_{\alpha} \dot{\beta} \bar{R}\right) \overline{\mathcal{D}}_{\dot{\beta}}+2 \mathrm{i}\left(\bar{\xi}^{\dot{\beta}} \delta_{\alpha}{ }^{\beta}-\frac{\mathrm{i}}{4} \mathcal{D}_{\alpha} \xi^{\beta \dot{\beta}}\right) \mathcal{D}_{\beta \dot{\beta}} \\
& -\left(\mathcal{D}_{\alpha} K^{\beta \gamma}+4 \bar{R} \delta_{\alpha}{ }^{(\beta} \xi^{\gamma)}-\frac{\mathrm{i}}{2} \delta_{\alpha}{ }^{(\beta} \xi^{\gamma) \dot{\gamma}} \overline{\mathcal{D}}_{\dot{\gamma}} \bar{R}-\frac{\mathrm{i}}{2} \xi_{\alpha \dot{\alpha}} \mathcal{D}^{(\beta} G^{\gamma) \dot{\alpha}}-2 \delta_{\alpha}{ }^{(\beta} \mathcal{D}^{\gamma)} \Sigma\right) M_{\beta \gamma} \\
& -\left(\mathcal{D}_{\alpha} \bar{K}^{\dot{\beta} \dot{\gamma}}+\mathrm{i} \xi_{\alpha \dot{\alpha}} \bar{W}^{\dot{\alpha} \dot{\beta} \dot{\gamma}}+\frac{\mathrm{i}}{6} \xi_{\alpha}{ }^{(\dot{\beta}} \bar{X}^{\dot{\gamma})}\right) \bar{M}_{\dot{\beta} \dot{\gamma}} \\
& -\mathrm{i}\left(\mathcal{D}_{\alpha} \rho+\frac{1}{4} \xi_{\alpha \dot{\alpha}} \bar{X}^{\dot{\alpha}}+\frac{3 \mathrm{i}}{2} \mathcal{D}_{\alpha} \Sigma\right) \mathbb{A} .
\end{aligned}
$$

Setting this to zero, we can read off the necessary conditions on our gauge and super-Weyl parameters for $\xi$ to be conformal Killing. These conditions can be split into two types. The first type provides expressions for the transformation parameters in terms of $\xi$

$$
\begin{aligned}
\xi^{\alpha} & =-\frac{\mathrm{i}}{8} \overline{\mathcal{D}}_{\dot{\alpha}} \xi^{\alpha \dot{\alpha}} \\
K_{\alpha \beta}[\xi] & =\mathcal{D}_{(\alpha} \xi_{\beta)}-\frac{\mathrm{i}}{2} \xi_{(\alpha} \dot{\alpha} G_{\beta) \dot{\alpha}} \\
\rho[\xi] & =-\frac{\mathrm{i}}{4}\left(\mathcal{D}^{\alpha} \xi_{\alpha}-\overline{\mathcal{D}}_{\dot{\alpha}} \bar{\xi}^{\dot{\alpha}}\right)-\frac{1}{4} G^{\alpha \dot{\alpha}} \xi_{\alpha \dot{\alpha}}, \\
\Sigma[\xi] & =-\frac{1}{2}\left(\mathcal{D}^{\alpha} \xi_{\alpha}+\overline{\mathcal{D}}_{\dot{\alpha}} \bar{\xi}^{\dot{\alpha}}\right) .
\end{aligned}
$$

The second type yields expressions for the spinor covariant derivatives of the parameters in terms of the original parameters and $\mathcal{D}_{\alpha} \Sigma[\xi]$, including the following:

$$
\begin{aligned}
\mathcal{D}_{\alpha} \xi_{\beta}= & \varepsilon_{\alpha \beta}\left(\frac{\mathrm{i}}{4} G^{\gamma \dot{\gamma}} \xi_{\gamma \dot{\gamma}}+\mathrm{i} \rho[\xi]-\frac{1}{2} \Sigma[\xi]\right)+K_{\alpha \beta}[\xi]+\frac{\mathrm{i}}{2} \xi_{(\alpha}{ }^{\dot{\alpha}} G_{\beta) \dot{\alpha}}, \\
\mathcal{D}_{\alpha} \bar{\xi}_{\dot{\beta}}= & -\frac{\mathrm{i}}{2} \xi_{\alpha \dot{\beta}} \bar{R} \\
\mathcal{D}_{\alpha} \xi_{\beta \dot{\beta}}= & 4 \mathrm{i} \varepsilon_{\alpha \beta} \bar{\xi}_{\dot{\beta}}, \\
\mathcal{D}_{\alpha} K^{\beta \gamma}[\xi]= & 2 \delta_{\alpha}{ }^{(\beta} \mathcal{D}^{\gamma)} \Sigma[\xi]-4 \delta_{\alpha}{ }^{(\beta} \xi^{\gamma)} \bar{R}+\frac{\mathrm{i}}{2} \delta_{\alpha}{ }^{(\beta} \xi^{\gamma) \dot{\gamma}} \overline{\mathcal{D}}_{\dot{\gamma}} \bar{R} \\
& +\frac{\mathrm{i}}{2} \xi_{\alpha \dot{\alpha}} \mathcal{D}^{(\beta} G^{\gamma) \dot{\alpha}} \\
\mathcal{D}_{\alpha} \bar{K}^{\dot{\beta} \dot{\gamma}}[\xi]= & -\mathrm{i} \xi_{\alpha \dot{\alpha}} \bar{W}^{\dot{\alpha} \dot{\beta} \dot{\gamma}}-\frac{\mathrm{i}}{6} \xi_{\alpha}{ }^{(\dot{\beta}} \bar{X}^{\dot{\gamma})} \\
\mathcal{D}_{\alpha} \rho[\xi]= & -\frac{1}{4} \xi_{\alpha \dot{\alpha}} \bar{X}^{\dot{\alpha}}-\frac{3 \mathrm{i}}{2} \mathcal{D}_{\alpha} \Sigma[\xi] .
\end{aligned}
$$

The relations (3.3) tell us that all the parameters are completely determined in terms of $\xi^{a}$ and its covariant derivatives. As will be shown below, the relations (3.4) imply that the the superalgebra of conformal Killing supervector fields is finite dimensional.

The above analysis shows that $\xi=\xi^{A} E_{A}$ is a conformal Killing supervector field if it has the form

$$
\xi^{A}=\left(\xi^{a},-\frac{\mathrm{i}}{8} \overline{\mathcal{D}}_{\dot{\beta}} \xi^{\alpha \dot{\beta}},-\frac{\mathrm{i}}{8} \mathcal{D}^{\beta} \xi_{\beta \dot{\alpha}}\right)
$$


where $\xi^{a}$ obeys the equation

$$
\mathcal{D}_{(\alpha} \xi_{\beta) \dot{\beta}}=0 \quad \Longleftrightarrow \quad \overline{\mathcal{D}}_{(\dot{\alpha}} \xi_{\beta \dot{\beta})}=0
$$

in accordance with (3.4c). Provided the equation (3.5b) and definitions (3.3) hold, one may check that all the conditions (3.4) are satisfied. Equation (3.5b) also implies that $\xi^{a}$ is covariantly linear,

$$
\left(\mathcal{D}^{2}+2 \bar{R}\right) \xi^{a}=0
$$

as well as the ordinary conformal Killing equation

$$
\mathcal{D}_{(a} \xi_{b)}=\frac{1}{4} \eta_{a b} \mathcal{D}^{c} \xi_{c} \quad \Longleftrightarrow \quad \mathcal{D}_{\left(\alpha \left(\dot{\alpha} \xi_{\beta) \dot{\beta})}\right.\right.}=0
$$

Due to the relation $\left\{\mathcal{D}_{\alpha}, \overline{\mathcal{D}}_{\dot{\alpha}}\right\}=-2 \mathrm{i} \mathcal{D}_{\alpha \dot{\alpha}}$, the equation (3.1) with $A=a$ is automatically satisfied once (3.1) with $A=\alpha$ holds. Still the implications of the equation (3.1) with $A=a$ prove to be very useful for computations, and we spell them out here:

$$
\begin{aligned}
& \mathcal{D}_{\alpha \dot{\alpha}} \xi^{\beta}=-\mathrm{i} \xi_{\alpha} G^{\beta}{ }_{\dot{\alpha}}-\mathrm{i} \delta_{\alpha}{ }^{\beta} \bar{\xi}_{\dot{\alpha}} R-\frac{1}{4} \xi_{\alpha}{ }^{\dot{\beta}} \overline{\mathcal{D}}_{(\dot{\alpha}} G^{\beta}{ }_{\dot{\beta})}+\frac{1}{4} \delta_{(\alpha}{ }^{\beta} \xi^{\gamma}{ }_{\dot{\alpha}} \mathcal{D}_{\gamma)} R \\
& +\frac{1}{2} \xi^{\gamma}{ }_{\alpha} W_{\alpha \gamma}{ }^{\beta}+\frac{1}{12} \delta_{(\alpha}{ }^{\beta} X_{\gamma)} \xi^{\gamma} \dot{\alpha}+\mathrm{i} \delta_{\alpha}{ }^{\beta} \overline{\mathcal{D}}_{\dot{\alpha}} \Sigma[\xi], \\
& \left.\mathcal{D}_{\alpha \dot{\alpha}} \xi^{\beta \dot{\beta}}=-\mathrm{i} \delta_{(\dot{\alpha}}{ }^{\dot{\beta}} \xi_{\alpha}{ }^{\dot{\gamma}} G^{\beta} \dot{\gamma}\right)+\mathrm{i} \delta_{(\alpha}{ }^{\beta} \xi^{\gamma} \dot{\alpha} G_{\gamma)}{ }^{\dot{\beta}}-2 \delta_{\dot{\alpha}}{ }^{\dot{\beta}} K_{\alpha}{ }^{\beta}[\xi]-2 \delta_{\alpha}{ }^{\beta} \bar{K}_{\dot{\alpha}}{ }^{\dot{\beta}}[\xi] \\
& -2 \delta_{\alpha}{ }^{\beta} \delta_{\dot{\alpha}}^{\dot{\beta}} \Sigma[\xi] \text {, } \\
& \mathcal{D}_{\alpha \dot{\alpha}} K^{\beta \gamma}[\xi]=\mathrm{i} \xi_{\alpha} \mathcal{D}^{(\beta} G^{\gamma)}{ }_{\dot{\alpha}}+\mathrm{i} \delta_{\alpha}{ }^{(\beta} \xi^{\gamma)} \overline{\mathcal{D}}_{\dot{\alpha}} \bar{R}+\frac{\mathrm{i}}{3} \delta_{\alpha}{ }^{(\beta} \bar{\xi}_{\dot{\alpha}} X^{\gamma)}-2 \mathrm{i} \bar{\xi}_{\dot{\alpha}} W_{\alpha}{ }^{\beta \gamma} \\
& +\frac{1}{4} \xi_{\alpha}{ }^{\dot{\beta}} \overline{\mathcal{D}}_{(\dot{\alpha}} \mathcal{D}^{(\beta} G^{\gamma)}{ }_{\dot{\beta})}+\frac{1}{8} \delta_{\alpha}{ }^{(\beta} \xi^{\gamma)}{ }_{\dot{\alpha}}\left(\mathcal{D}^{2}-8 \bar{R}\right) R+\frac{1}{2} \xi^{\lambda}{ }_{\alpha} \mathcal{D}_{(\alpha} W_{\lambda)}{ }^{\beta \gamma} \\
& -\frac{1}{12} \xi^{\lambda} \delta_{(\alpha}{ }^{(\beta} \mathcal{D}_{\lambda)} X^{\gamma)}+\mathrm{i} \delta_{\alpha}{ }^{(\beta} \overline{\mathcal{D}}_{\dot{\alpha}} \mathcal{D}^{\gamma)} \Sigma[\xi], \\
& \mathcal{D}_{\alpha \dot{\alpha}} \rho[\xi]=\frac{1}{2} \bar{\xi}_{\dot{\alpha}} X_{\alpha}-\frac{1}{2} \xi_{\alpha} \bar{X}_{\dot{\alpha}}+\frac{\mathrm{i}}{8} \xi_{\alpha}{ }^{\dot{\beta}} \overline{\mathcal{D}}_{(\dot{\alpha}} \bar{X}_{\dot{\beta})}+\frac{\mathrm{i}}{8} \xi^{\beta} \mathcal{D}_{(\alpha} X_{\beta)} \\
& -\frac{3}{4}\left[\mathcal{D}_{\alpha}, \overline{\mathcal{D}}_{\dot{\alpha}}\right] \Sigma[\xi] \text {. }
\end{aligned}
$$

We emphasise once more that these identities may be derived by making use of (3.4).

\subsection{The superconformal algebra}

It follows from (3.1) that commuting two superconformal transformations of $\left(\mathcal{M}^{4 \mid 4}, \mathcal{D}\right)$ results in another transformation of the same type,

$$
\begin{aligned}
{\left[\delta_{\mathcal{K}\left[\xi_{2}\right]}+\delta_{\Sigma\left[\xi_{2}\right]}, \delta_{\mathcal{K}\left[\xi_{1}\right]}+\delta_{\Sigma\left[\xi_{1}\right]}\right] \mathcal{D}_{A} } & =\left(\delta_{\mathcal{K}\left[\xi_{3}\right]}+\delta_{\Sigma\left[\xi_{3}\right]}\right) \mathcal{D}_{A}=0 \\
\mathcal{K}\left[\xi_{3}\right] & :=\left[\mathcal{K}\left[\xi_{2}\right], \mathcal{K}\left[\xi_{1}\right]\right]
\end{aligned}
$$

This means that the set of all conformal Killing supervector fields forms a Lie superalgebra, the superconformal algebra of $\left(\mathcal{M}^{4 \mid 4}, \mathcal{D}\right)$. 
It is of interest to derive the explicit expressions for $\Sigma\left[\xi_{3}\right]$ and $\xi_{3}^{a}$ in terms of $\xi_{1}^{a}$ and $\xi_{2}^{a}$. A routine calculation gives

$$
\left[\mathcal{K}\left[\xi_{3}\right], \mathcal{D}_{A}\right]+\left[\mathcal{K}\left[\xi_{2}\right], \delta_{\Sigma\left[\xi_{1}\right]} \mathcal{D}_{A}\right]-\left[\mathcal{K}\left[\xi_{1}\right], \delta_{\Sigma\left[\xi_{2}\right]} \mathcal{D}_{A}\right]=0
$$

Specialising here to the $A=\alpha$ case and extracting the super-Weyl parameter, we find

$$
\Sigma\left[\xi_{3}\right]=\xi_{2}^{A} \mathcal{D}_{A} \Sigma\left[\xi_{1}\right]-\xi_{1}^{A} \mathcal{D}_{A} \Sigma\left[\xi_{2}\right] .
$$

For the vector component $\xi_{3}^{a}$ we obtain

$$
\xi_{3}^{\alpha \dot{\alpha}}=-\frac{1}{2} \xi_{1}^{\beta \dot{\beta}} \mathcal{D}_{\beta \dot{\beta}} \xi_{2}^{\alpha \dot{\alpha}}-\frac{\mathrm{i}}{16} \overline{\mathcal{D}}_{\dot{\beta}} \xi_{1}^{\alpha \dot{\beta}} \mathcal{D}_{\beta} \xi_{2}^{\beta \dot{\alpha}}+\frac{\mathrm{i}}{2} \xi_{1}^{\alpha \dot{\beta}} \xi_{2}^{\beta \dot{\alpha}} G_{\beta \dot{\beta}}-(1 \leftrightarrow 2) .
$$

One may check that $\xi_{3}^{\alpha \dot{\alpha}}$ obeys the superconformal Killing equation (3.5b).

The superconformal algebra of $\left(\mathcal{M}^{4 \mid 4}, \mathcal{D}\right)$ turns out to be finite dimensional, and its dimension does not exceed that of the $\mathcal{N}=1$ superconformal group $\mathrm{SU}(2,2 \mid 1)$. In order to prove this claim, we introduce the following set of parameters:

$$
\boldsymbol{\Xi}:=\left\{\xi^{A}, K^{\alpha \beta}[\xi], \bar{K}^{\dot{\alpha} \dot{\beta}}[\xi], \rho[\xi], \Sigma[\xi], \mathcal{D}_{A} \Sigma[\xi]\right\} .
$$

It is not difficult to demonstrate that $\mathcal{D}_{A} \boldsymbol{\Xi}$ is a linear combination of the elements of (3.13). Actually, it suffices to show that $\mathcal{D}_{\alpha} \boldsymbol{\Xi}$ satisfies this property, as the general case immediately follows. Due to the relations (3.4) and (3.8), we only need to analyse $\mathcal{D}_{\alpha} \mathcal{D}_{B} \Sigma[\xi]$. Direct calculations give

$$
\begin{aligned}
& \mathcal{D}_{\alpha} \mathcal{D}_{\beta} \Sigma[\xi]=\frac{1}{2} \varepsilon_{\alpha \beta} \mathcal{D}^{2} \Sigma=-\varepsilon_{\alpha \beta}\left((\Sigma[\xi]-2 \mathrm{i} \rho[\xi]) \bar{R}+\xi^{c} \mathcal{D}_{c} R+\bar{\xi} \overline{\mathcal{D}} \bar{R}\right), \\
& \mathcal{D}_{\alpha} \overline{\mathcal{D}}_{\dot{\beta}} \Sigma[\xi]=-\mathrm{i} \mathcal{D}_{\alpha \dot{\beta}} \Sigma[\xi]+\frac{1}{2} \bar{\xi}^{\dot{\gamma}} \overline{\mathcal{D}}_{\dot{\gamma}} G_{\alpha \dot{\beta}}-\frac{1}{2} \xi^{\gamma} \mathcal{D}_{\gamma} G_{\alpha \dot{\beta}} \\
& -\frac{1}{4} \xi_{\alpha}{ }^{\dot{\gamma}} \mathcal{D}^{\gamma}{ }_{(\dot{\beta}} G_{\gamma \dot{\gamma})}+\frac{1}{4} \xi^{\gamma} \mathcal{D}_{(\alpha}{ }^{\dot{\gamma}} G_{\gamma) \dot{\gamma}}+\frac{1}{4} \xi^{\gamma \dot{\gamma}} \mathcal{D}_{\alpha \dot{\beta}} G_{\gamma \dot{\gamma}} \\
& -\frac{1}{2} K_{\alpha}^{\gamma}[\xi] G_{\gamma \dot{\beta}}-\frac{1}{2} \bar{K}_{\dot{\beta}}^{\dot{\gamma}}[\xi] G_{\alpha \dot{\gamma}}-\frac{1}{2} G_{\alpha \dot{\beta}} \Sigma[\xi] \text {, } \\
& \mathcal{D}_{\alpha} \mathcal{D}_{\beta \dot{\beta}} \Sigma[\xi]=\varepsilon_{\alpha \beta}\left[\left(\frac{\mathrm{i}}{8} \delta^{\dot{\alpha}}{ }_{\dot{\beta}} \Sigma[\xi]+\frac{1}{12} \delta^{\dot{\alpha}}{ }_{\dot{\beta}} \rho[\xi]+\frac{\mathrm{i}}{12} \bar{K}^{\dot{\alpha}}{ }_{\dot{\beta}}[\xi]\right)\left(\bar{X}_{\dot{\alpha}}+3 \overline{\mathcal{D}}_{\dot{\alpha}} \bar{R}\right)+\mathrm{i} \bar{R}_{\bar{\beta}} \Sigma[\xi]\right. \\
& -\frac{\mathrm{i}}{2} G_{\dot{\beta}}^{\gamma} \mathcal{D}_{\gamma} \Sigma[\xi]-\frac{\mathrm{i}}{12} \bar{\xi}^{\dot{\alpha}} \overline{\mathcal{D}}_{(\dot{\alpha}} \bar{X}_{\dot{\beta})}+\frac{7 \mathrm{i}}{24} \bar{\xi}_{\dot{\beta}} \mathcal{D} X-\frac{\mathrm{i}}{8} \bar{\xi}_{\dot{\beta}} \overline{\mathcal{D}}^{2} \bar{R}+\frac{3 \mathrm{i}}{4} \xi^{\gamma} G_{\gamma \dot{\beta}} \bar{R} \\
& \left.-\frac{\mathrm{i}}{8} \xi^{\gamma} \mathcal{D}^{2} G_{\gamma \dot{\beta}}+\frac{1}{12} \xi^{\gamma \dot{\alpha}} \mathcal{D}_{\gamma} \overline{\mathcal{D}}_{(\dot{\alpha}} \bar{X}_{\dot{\beta})}-\frac{1}{48} \xi_{\dot{\beta}}^{\gamma} \mathcal{D}^{2} X_{\gamma}-\frac{\mathrm{i}}{8} \xi^{\gamma \dot{\alpha}} \mathcal{D}_{\gamma \dot{\alpha}}\left(\overline{\mathcal{D}}_{\dot{\beta}} \bar{R}-\bar{X}_{\dot{\beta}}\right)\right] \\
& +\left[6\left(\frac{\mathrm{i}}{8} \delta^{\dot{\alpha}}{ }_{\dot{\beta}} \Sigma[\xi]+\frac{1}{12} \delta_{\dot{\beta}}^{\dot{\alpha}} \rho[\xi]+\frac{\mathrm{i}}{12} \bar{K}_{\dot{\beta}}^{\dot{\alpha}}[\xi]\right) \mathcal{D}_{(\alpha} G_{\beta) \dot{\alpha}}+\mathrm{i} K_{(\alpha}^{\gamma}[\xi] \mathcal{D}_{\beta)} G_{\gamma \dot{\beta}}\right. \\
& -\frac{\mathrm{i}}{2} K_{\alpha \beta}[\xi]\left(\overline{\mathcal{D}}_{\dot{\beta}} \bar{R}-\bar{X}_{\dot{\beta}}\right)-\frac{\mathrm{i}}{2} \bar{\xi}^{\dot{\alpha}} \overline{\mathcal{D}}_{\dot{\alpha}} \mathcal{D}_{(\alpha} G_{\beta) \dot{\beta}}-\frac{\mathrm{i}}{4} \xi_{(\alpha}\left(\mathcal{D}^{2}+2 \bar{R}\right) G_{\beta) \dot{\beta}} \\
& \left.-\frac{1}{12} \xi_{(\alpha(\dot{\alpha}} G_{\beta) \dot{\beta})} \bar{X}^{\dot{\alpha}}-\frac{1}{12} \xi_{(\alpha \dot{\alpha}} G_{\beta)}{ }^{\dot{\alpha}} \bar{X}_{\dot{\beta}}-\frac{\mathrm{i}}{4} \xi^{\gamma \dot{\alpha}} \mathcal{D}_{\gamma \dot{\alpha}} \mathcal{D}_{(\alpha} G_{\beta) \dot{\beta}}\right]
\end{aligned}
$$

Thus, we have demonstrated that the superconformal algebra is finite dimensional. 


\subsection{Conformally related superspaces}

Let $\left(\mathcal{M}^{4 \mid 4}, \mathcal{D}\right)$ and $\left(\mathcal{M}^{4 \mid 4}, \hat{\mathcal{D}}\right)$ be two supergravity backgrounds. We say that the two superspaces are conformally related if their covariant derivatives $\hat{\mathcal{D}}_{A}$ and $\mathcal{D}_{A}$ are related to each other by a finite super-Weyl transformation (2.17),

$$
\begin{aligned}
\hat{\mathcal{D}}_{\alpha} & =\mathrm{e}^{\frac{1}{2} \Sigma}\left(\mathcal{D}_{\alpha}+2 \mathcal{D}^{\beta} \Sigma M_{\beta \alpha}+\frac{3}{2} \mathcal{D}_{\alpha} \Sigma \mathbb{A}\right), \\
\hat{\overline{\mathcal{D}}}_{\dot{\alpha}} & =\mathrm{e}^{\frac{1}{2} \Sigma}\left(\overline{\mathcal{D}}_{\dot{\alpha}}+2 \overline{\mathcal{D}}^{\dot{\beta}} \Sigma \bar{M}_{\dot{\beta} \dot{\alpha}}-\frac{3}{2} \overline{\mathcal{D}}_{\dot{\alpha}} \Sigma \mathbb{A}\right), \\
\hat{\mathcal{D}}_{\alpha \dot{\alpha}} & =\frac{\mathrm{i}}{2}\left\{\hat{\mathcal{D}}_{\alpha}, \hat{\overline{\mathcal{D}}}_{\dot{\alpha}}\right\} .
\end{aligned}
$$

These superspaces prove to have the same conformal Killing supervector fields,

$$
\xi=\xi^{A} E_{A}=\hat{\xi}^{A} \hat{E}_{A},
$$

where the components $\hat{\xi}^{A}$ are given by

$$
\hat{\xi}^{\alpha \dot{\alpha}}=\mathrm{e}^{-\Sigma} \xi^{\alpha \dot{\alpha}}, \quad \hat{\xi}^{\alpha}=\mathrm{e}^{-\frac{1}{2} \Sigma}\left(\xi^{\alpha}+\frac{\mathrm{i}}{2} \xi^{\alpha \dot{\beta}} \overline{\mathcal{D}}_{\dot{\beta}} \Sigma\right) .
$$

The transformed supervector field $\hat{\xi}^{A}$ also satisfies (3.5) (in the new basis), thus it is a conformal Killing vector

$$
\hat{\xi}^{A}=\left(\hat{\xi}^{a},-\frac{\mathrm{i}}{8} \hat{\overline{\mathcal{D}}}_{\dot{\beta}} \hat{\xi}^{\alpha \dot{\beta}},-\frac{\mathrm{i}}{8} \hat{\mathcal{D}}^{\beta} \xi_{\beta \dot{\alpha}}\right), \quad \hat{\mathcal{D}}_{(\alpha} \hat{\xi}_{\beta) \dot{\beta}}=0
$$

One can relate the remaining parameters generating conformal isometries in each geometry in a simple way

$$
\begin{aligned}
\Sigma[\hat{\xi}] & =\Sigma[\xi]-\xi^{A} \mathcal{D}_{A} \Sigma[\xi] \\
K_{\alpha \beta}[\hat{\xi}] & =K_{\alpha \beta}[\xi]+2 \mathcal{D}_{(\alpha} \Sigma \xi_{\beta)}+\frac{\mathrm{i}}{2} \overline{\mathcal{D}}_{\dot{\alpha}} \mathcal{D}_{(\alpha} \Sigma \xi_{\beta)}{ }^{\dot{\alpha}}, \\
\rho[\hat{\xi}] & =\rho[\xi]+\frac{3 \mathrm{i}}{2} \mathcal{D}^{\alpha} \Sigma \xi_{\alpha}-\frac{3 \mathrm{i}}{2} \overline{\mathcal{D}}_{\dot{\alpha}} \Sigma \bar{\xi}^{\dot{\alpha}}-\frac{3}{8}\left[\mathcal{D}^{\alpha}, \overline{\mathcal{D}}^{\dot{\alpha}}\right] \Sigma \xi_{\alpha \dot{\alpha}} .
\end{aligned}
$$

It then follows that the gauge transformation is identical in these two geometries $\mathcal{K}[\xi]=$ $\mathcal{K}[\hat{\xi}]$; it is a super-Weyl invariant operator.

\subsection{Superconformal field theory}

Let $\varphi^{i}$ be the dynamical superfield variables describing a matter system coupled to conformal supergravity. The matter action is required to be invariant under the super-Weyl transformations (2.12) accompanied by certain transformations of the matter superfields of the form

$$
\delta_{\Sigma} \varphi^{i}=\Delta_{(i)} \Sigma \varphi^{i}
$$

where $\Delta_{(i)}$ denotes the dimension of $\varphi^{i}$. In general, the matter action includes two terms

$$
S=\int \mathrm{d}^{4} x \mathrm{~d}^{2} \theta \mathrm{d}^{2} \bar{\theta} E \mathcal{L}+\left\{\int \mathrm{d}^{4} x \mathrm{~d}^{2} \theta \mathcal{E} \mathcal{L}_{\mathrm{c}}+\text { c.c. }\right\}, \quad E^{-1}=\operatorname{Ber}\left(E_{A}{ }^{M}\right),
$$


with $\mathcal{E}$ being the so-called chiral density. Here the full superspace Lagrangian $\mathcal{L}$ is a primary real scalar superfield of dimension +2 , while $\mathcal{L}_{\mathrm{c}}$ is a primary covariantly chiral superfield, $\overline{\mathcal{D}}_{\dot{\alpha}} \mathcal{L}_{\mathrm{c}}=0$, of dimension +3 ,

$$
\delta_{\Sigma} \mathcal{L}=2 \Sigma \mathcal{L}, \quad \delta_{\Sigma} \mathcal{L}_{\mathrm{c}}=3 \Sigma \mathcal{L}_{\mathrm{c}}
$$

It should be pointed out that the full superspace measure $E$ and the chiral density $\mathcal{E}$ have the following super-Weyl transformation laws

$$
\delta_{\Sigma} E=-2 \Sigma E, \quad \delta_{\Sigma} \mathcal{E}=-3 \Sigma \mathcal{E} .
$$

The chiral density can be naturally defined using the prepotential solution of the supergravity constraints given in [37]. It can also be obtained using the general formalism of integrating out fermionic dimensions, which was developed in [63]. Probably the simplest definition of the chiral action

$$
S_{\mathrm{c}}=\int \mathrm{d}^{4} x \mathrm{~d}^{2} \theta \mathcal{E} \mathcal{L}_{\mathrm{c}}
$$

is described in appendix A. The full superspace action can be represented as an integral over the chiral subspace,

$$
\int \mathrm{d}^{4} x \mathrm{~d}^{2} \theta \mathrm{d}^{2} \bar{\theta} E \mathcal{L}=-\frac{1}{4} \int \mathrm{d}^{4} x \mathrm{~d}^{2} \theta \mathcal{E}\left(\overline{\mathcal{D}}^{2}-4 R\right) \mathcal{L} .
$$

In the case of a fixed supergravity background, the matter action (3.21) is invariant under superconformal transformations of the form

$$
\delta_{\xi} \varphi^{i}=\mathcal{K}[\xi] \varphi^{i}+\Delta_{(i)} \Sigma[\xi] \varphi^{i}
$$

where $\xi^{A}$ is an arbitrary conformal Killing supervector field of the background curved superspace $\left(\mathcal{M}^{4 \mid 4}, \mathcal{D}\right)$.

An important example of a superconformal field theory in curved superspace is the massless Wess-Zumino model

$$
S[\phi, \bar{\phi}]=\int \mathrm{d}^{4} x \mathrm{~d}^{2} \theta \mathrm{d}^{2} \bar{\theta} E \bar{\phi} \phi+\left\{\frac{\lambda}{3 !} \int \mathrm{d}^{4} x \mathrm{~d}^{2} \theta \mathcal{E} \phi^{3}+\text { c.c. }\right\}, \quad \overline{\mathcal{D}}_{\dot{\alpha}} \phi=0,
$$

with $\lambda$ a coupling constant. Here the chiral scalar $\phi$ is primary and of dimension +1 .

\subsection{Superconformal sigma models}

A nontrivial example of a superconformal field theory on $\left(\mathcal{M}^{4 \mid 4}, \mathcal{D}\right)$ is a nonlinear sigma model. The target spaces of superconformal sigma models are Kähler cones [64]. Let us recall what this means. Consider a Kähler manifold $\left(\mathcal{N}, g_{\mu \nu}, J^{\mu}{ }_{\nu}\right)$, where $\mu, \nu=1, \ldots, 2 n$, and introduce local complex coordinates $\phi^{i}$ and their conjugates $\bar{\phi}^{\bar{i}}$, in which the complex structure $J_{\nu}^{\mu}$ is diagonal. It is called a Kähler cone [64] if it possesses a homothetic conformal Killing vector

$$
\chi=\chi^{i} \frac{\partial}{\partial \phi^{i}}+\bar{\chi}^{\bar{i}} \frac{\partial}{\partial \bar{\phi}^{\bar{i}}} \equiv \chi^{\mu} \frac{\partial}{\partial \varphi^{\mu}}
$$


with the following properties:

$$
\nabla_{\nu} \chi^{\mu}=\delta_{\nu}{ }^{\mu} \Longleftrightarrow \nabla_{j} \chi^{i}=\delta_{j}{ }^{i}, \quad \nabla_{\bar{j}} \chi^{i}=\partial_{\bar{j}} \chi^{i}=0,
$$

which show, in particular, that $\chi$ is holomorphic. In terms of the scalar field $K:=g_{i \bar{j}} \chi^{i} \bar{\chi}^{\bar{j}}$ on the target space, these properties imply that

$$
\chi_{i}=g_{i \bar{j}} \bar{\chi}^{\bar{j}}=\partial_{i} K, \quad g_{i \bar{j}}=\partial_{i} \partial_{\bar{j}} K,
$$

and therefore

$$
\chi^{i}(\phi) \partial_{i} K(\phi, \bar{\phi})=K(\phi, \bar{\phi}) \text {. }
$$

The real function $K(\phi, \bar{\phi})$ is a globally defined Kähler potential. Associated with $\chi$ is the $\mathrm{U}(1)$ Killing vector field

$$
V^{\mu}=J_{\nu}^{\mu} \chi^{\nu}, \quad \nabla_{\mu} V_{\nu}+\nabla_{\nu} V_{\mu}=0 .
$$

Local complex coordinates $\phi^{i}$ can always be chosen such that $\chi^{i}(\phi)=\phi^{i}$.

Consider the following nonlinear $\sigma$-model

$$
S=\int \mathrm{d}^{4} x \mathrm{~d}^{2} \theta \mathrm{d}^{2} \bar{\theta} E K(\phi, \bar{\phi}), \quad \overline{\mathcal{D}}_{\dot{\alpha}} \phi^{i}=0,
$$

where the action of the $\mathrm{U}(1)_{R}$ generator on $\phi^{i}$ is defined as

$$
\mathbb{A} \phi^{i}=\frac{2}{3} \chi^{i}(\phi) \text {. }
$$

The action is invariant under super-Weyl transformations

$$
\delta_{\Sigma} \phi^{i}=\Sigma \chi^{i}(\phi) .
$$

In the case of a fixed supergravity background, the matter action (3.21) is invariant under superconformal transformations of the form

$$
\delta_{\xi} \phi^{i}=\mathcal{K}[\xi] \phi^{i}+\Sigma[\xi] \chi^{i}(\phi),
$$

where $\xi^{A}$ is an arbitrary conformal Killing supervector field of the background curved superspace $\left(\mathcal{M}^{4 \mid 4}, \mathcal{D}\right)$.

\section{Conformal Killing tensor superfields}

As discussed in section 3, every conformal Killing supervector field $\xi^{A}$ of the background curved superspace $\left(\mathcal{M}^{4 \mid 4}, \mathcal{D}\right)$ is determined by its vector component $\xi^{a}$, which is real and constrained by

$$
\mathcal{D}_{(\alpha} \xi_{\beta) \dot{\beta}}=0 \quad \Longleftrightarrow \quad \overline{\mathcal{D}}_{(\dot{\alpha}} \xi_{\beta \dot{\beta})}=0 .
$$

It follows from (3.17) that $\xi_{\alpha \dot{\alpha}}$ has the super-Weyl transformation law

$$
\delta_{\Sigma} \xi_{\alpha \dot{\alpha}}=-\Sigma \xi_{\alpha \dot{\alpha}},
$$

which is uniquely determined by requiring equations (4.1) to be super-Weyl invariant. This construction admits nontrivial generalisations. 


\subsection{Definitions}

Let $m$ and $n$ be non-negative integers. A primary tensor superfield $\ell_{\alpha(m) \dot{\alpha}(n)}$ on $\left(\mathcal{M}^{4 \mid 4}, \mathcal{D}\right)$ is called conformal Killing if it obeys the constraints ${ }^{6}$

$$
\begin{aligned}
& \mathcal{D}_{\left(\alpha_{1}\right.} \ell_{\left.\alpha_{2} \ldots \alpha_{m+1}\right) \dot{\alpha}(n)}=0 \quad \Longrightarrow \quad\left(\mathcal{D}^{2}+2 m \bar{R}\right) \ell_{\alpha(m) \dot{\alpha}(n)}=0,
\end{aligned}
$$

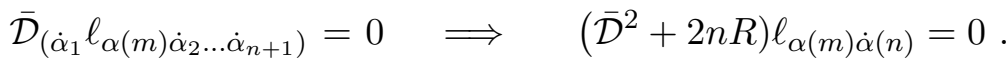

These conditions imply the following transformation properties:

$$
\begin{aligned}
\delta_{\Sigma} \ell_{\alpha(m) \dot{\alpha}(n)} & =-\frac{1}{2}(m+n) \sum \ell_{\alpha(m) \dot{\alpha}(n)}, \\
\mathbb{A} \ell_{\alpha(m) \dot{\alpha}(n)} & =-\frac{1}{3}(m-n) \ell_{\alpha(m) \dot{\alpha}(n)} .
\end{aligned}
$$

If $m=n$, then $\ell_{\alpha(n) \dot{\alpha}(n)}$ is neutral with respect to the $R$-symmetry group $\mathrm{U}(1)_{R}$, and therefore it is consistent to restrict $\ell_{\alpha(n) \dot{\alpha}(n)}$ to be real. Another special choice is $n=0$, in which case $\ell_{\alpha(m)}$ is covariantly chiral, $\overline{\mathcal{D}}_{\dot{\alpha}} \ell_{\alpha(m)}=0$.

The constraints (4.3) provide a natural generalisation of the concept of a conformal Killing tensor field $L_{\alpha(m) \dot{\alpha}(n)}$ on a curved spacetime $\mathcal{M}^{4}$ [65]. ${ }^{7}$ By definition, $L_{\alpha(m) \dot{\alpha}(n)}$ is a primary field which obeys the equation

$$
\nabla_{\left(\alpha_{1}\right.}{ }^{\left(\dot{\alpha}_{1}\right.} L_{\left.\alpha_{2} \ldots \alpha_{m+1}\right)}{ }^{\left.\dot{\alpha}_{2} \ldots \dot{\alpha}_{n+1}\right)}=0,
$$

where $\nabla_{\alpha \dot{\alpha}}$ is the torsion-free Lorentz-covariant derivative. The condition that $L_{\alpha(m) \dot{\alpha}(n)}$ is primary means that it changes homogeneously under a Weyl transformation

$$
\delta_{\sigma} \nabla_{a}=\sigma \nabla_{a}-\nabla^{b} \sigma M_{a b}
$$

with $\sigma(x)$ the Weyl parameter. The unique Weyl transformation law of $L_{\alpha(m) \dot{\alpha}(n)}$, which is compatible with the constraint (4.5), is

$$
\delta_{\sigma} L_{\alpha(m) \dot{\alpha}(n)}=-\frac{1}{2}(m+n) \sigma L_{\alpha(m) \dot{\alpha}(n)} .
$$

Given two conformal Killing tensor superfields $\ell_{\alpha(m) \dot{\alpha}(n)}$ and $\ell_{\alpha(p) \dot{\alpha}(q)}$ on $\left(\mathcal{M}^{4 \mid 4}, \mathcal{D}\right)$, their symmetric product

$$
\ell_{\alpha(m+p) \dot{\alpha}(n+q)}:=\ell_{\left(\alpha _ { 1 } \ldots \alpha _ { m } \left(\dot{\alpha}_{1} \ldots \dot{\alpha}_{n}\right.\right.} \ell_{\left.\left.\alpha_{m+1} \ldots \alpha_{m+p}\right) \dot{\alpha}_{n+1} \ldots \dot{\alpha}_{n+q}\right)},
$$

is also conformal Killing. This operation allows one to generate new conformal Killing tensor superfields from given ones.

Constraints (4.3) naturally occur in the framework of conformal higher-spin gauge supermultiplets [66, 67]. For $m \geq n>0$ such a supermultiplet is described by an unconstrained primary prepotential $\Upsilon_{\alpha(m) \dot{\alpha}(n)}$ defined modulo gauge transformations

$$
\delta_{\Lambda, \zeta} \Upsilon_{\alpha(m) \dot{\alpha}(n)}=\mathcal{D}_{\left(\alpha_{1}\right.} \zeta_{\left.\alpha_{2} \ldots \alpha_{m}\right) \dot{\alpha}_{1} \ldots \dot{\alpha}_{n}}+\overline{\mathcal{D}}_{\left(\dot{\alpha}_{1}\right.} \Lambda_{\left.\alpha_{1} \ldots \alpha_{m} \dot{\alpha}_{2} \ldots \dot{\alpha}_{n}\right)},
$$

\footnotetext{
${ }^{6}$ These constraints can be naturally lifted to the conformal superspace of [40].

${ }^{7}$ Penrose and Rindler [65] called $L_{\alpha(m) \dot{\alpha}(n)}$ a Killing spinor.
} 
with unconstrained primary gauge parameters $\zeta_{\alpha(m-1) \dot{\alpha}(n)}$ and $\Lambda_{\alpha(m) \dot{\alpha}(n-1)}$. In the $m>$ $n=0$ case, the conformal gauge supermultiplet is described by an unconstrained primary prepotential $\Upsilon_{\alpha(m)}$ defined modulo gauge transformations

$$
\delta_{\zeta, \lambda} \Upsilon_{\alpha(m)}=\mathcal{D}_{\left(\alpha_{1}\right.} \zeta_{\left.\alpha_{2} \ldots \alpha_{m}\right)}+\lambda_{\alpha(m)}, \quad \overline{\mathcal{D}}_{\dot{\beta}} \lambda_{\alpha(m)}=0
$$

Now, if we look for special gauge parameters $\zeta_{\alpha(m-1) \dot{\alpha}(n)}$ and $\Lambda_{\alpha(m) \dot{\alpha}(n-1)}$ such that the variation (4.9) vanishes, $\delta_{\Lambda, \zeta} \Upsilon_{\alpha(m) \dot{\alpha}(n)}=0$, then $\ell_{\alpha(m) \dot{\alpha}(n)}:=\mathcal{D}_{\left(\alpha_{1}\right.} \zeta_{\left.\alpha_{2} \ldots \alpha_{m}\right) \dot{\alpha}_{1} \ldots \dot{\alpha}_{n}}$ is a solution to the constraints (4.3).

A higher-spin interpretation exists also for the conformal Killing tensors (4.5). We recall that a conformal higher-spin gauge field $h_{\alpha(m+1) \dot{\alpha}(n+1)}$ is a primary field defined modulo gauge transformations [68]

$$
\delta_{\lambda} h_{\alpha(m+1) \dot{\alpha}(n+1)}=\nabla_{\left(\alpha _ { 1 } \left(\dot{\alpha}_{1}\right.\right.} \lambda_{\left.\left.\alpha_{2} \ldots \alpha_{m+1}\right) \dot{\alpha}_{2} \ldots \dot{\alpha}_{n+1}\right)},
$$

where the gauge parameter $\lambda_{\alpha(m) \dot{\alpha}(n)}$ is also primary. The conformal Killing tensors (4.5) correspond to those values of the gauge parameter $\lambda_{\alpha(m) \dot{\alpha}(n)}$ which leave the gauge field invariant, $\delta_{L} h_{\alpha(m+1) \dot{\alpha}(n+1)}=0$.

The importance of the conformal Killing superfields $\ell_{\alpha(m) \dot{\alpha}(n)}$ introduced is that they generate symmetries of dynamical systems on $\left(\mathcal{M}^{4 \mid 4}, \mathcal{D}\right)$. We have seen that the $\mathcal{N}=1$ superconformal transformations are described by $\ell_{\alpha \dot{\alpha}}$. In the next subsection, we introduce various important conformal supercurrents and describe their interplay with conformal Killing tensor superfields. Following this, in sections 4.3 and 4.4 we show that extended superconformal transformations are formulated in terms of $\ell_{\alpha}$ and its conjugate. Then in section 4.5, it will be demonstrated that higher-rank analogues of $\ell_{\alpha \dot{\alpha}}$, the conformal Killing tensor superfields $\ell_{\alpha(n) \dot{\alpha}(n)}$, generate symmetries of the massless Wess-Zumino operator.

\subsection{Conserved current supermultiplets}

When considering conformal field theories on $\mathbb{R}^{d-1,1}$, a well-known procedure exists to generate conserved conformal currents by making use of a symmetric, traceless and conserved energy-momentum tensor $T^{a b}$

$$
T^{a b}=T^{b a}, \quad \eta_{a b} T^{a b}=0, \quad \partial_{b} T^{a b}=0,
$$

with $\eta_{a b}$ the Minkowski metric. Given a conformal Killing vector field $\xi=\xi^{a} \partial_{a}$,

$$
\partial_{a} \xi_{b}+\partial_{b} \xi_{a}=\frac{2}{d} \eta_{a b} \partial_{c} \xi^{c}
$$

the following vector field

$$
j^{a}[\xi]=T^{a b} \xi_{b}
$$

is conserved, $\partial_{a} j^{a}=0$. The construction is naturally generalised to a curved space. It also has a higher-spin extension [42]. Here we will present supersymmetric extensions of these constructions building, in part, on the earlier work [69]. 
Let $m$ and $n$ be positive integers. A primary tensor superfield $J^{\alpha(m) \dot{\alpha}(n)}$ on $\left(\mathcal{M}^{4 \mid 4}, \mathcal{D}\right)$ is called a conformal supercurrent of valence $(m, n)$ if it obeys the constraints

$$
\begin{array}{lll}
\mathcal{D}_{\beta} J^{\beta \alpha(m-1) \dot{\alpha}(n)}=0 & \Longrightarrow & \left(\mathcal{D}^{2}-2(m+2) \bar{R}\right) J^{\alpha(m) \dot{\alpha}(n)}=0 \\
\overline{\mathcal{D}}_{\dot{\beta}} J^{\alpha(m) \dot{\beta} \dot{\alpha}(n-1)}=0 & \Longrightarrow & \left(\overline{\mathcal{D}}^{2}-2(n+2) R\right) J^{\alpha(m) \dot{\alpha}(n)}=0
\end{array}
$$

These conditions imply the following superconformal transformation properties:

$$
\begin{aligned}
\delta_{\Sigma} J^{\alpha(m) \dot{\alpha}(n)} & =\left(2+\frac{1}{2}(m+n)\right) \Sigma J^{\alpha(m) \dot{\alpha}(n)}, \\
\mathbb{A} J^{\alpha(m) \dot{\alpha}(n)} & =\frac{1}{3}(m-n) J^{\alpha(m) \dot{\alpha}(n)}
\end{aligned}
$$

If $m=n$, then $J^{\alpha(n) \dot{\alpha}(n)}$ is neutral with respect to the $R$-symmetry group $\mathrm{U}(1)_{R}$, and therefore it is consistent to restrict $J^{\alpha(n) \dot{\alpha}(n)}$ to be real. The $m=n=1$ case corresponds to the ordinary conformal supercurrent [70]. The case $m=n>1$ was first described in Minkowski superspace in [71] (see also [58, 66]) and extended to AdS superspace in [72].

In the case $m>n=0$, the constraints (4.15) should be replaced with

$$
\begin{aligned}
\mathcal{D}_{\beta} J^{\beta \alpha(m-1)} & =0 \quad \Longrightarrow \quad\left(\mathcal{D}^{2}-2(m+2) \bar{R}\right) J^{\alpha(m)}=0, \\
\left(\overline{\mathcal{D}}^{2}-4 R\right) J^{\alpha(m)} & =0
\end{aligned}
$$

The superconformal transformation properties of $J^{\alpha(m)}$ are obtained from (4.16) by setting $n=0$. The case $n=1$ was first considered in [73], where it was shown that the spinor supercurrent $J^{\alpha}$ naturally originates from the reduction of the conformal $\mathcal{N}=2$ supercurrent [74] to $\mathcal{N}=1$ superspace.

Finally, for $m=0$ the constraints (4.17) should be replaced with

$$
\begin{aligned}
\left(\mathcal{D}^{2}-4 \bar{R}\right) J & =0, \\
\left(\overline{\mathcal{D}}^{2}-4 R\right) J & =0 .
\end{aligned}
$$

This is the flavour current supermultiplet [75].

Let $J^{\alpha(m) \dot{\alpha}(n)}$ be a conformal supercurrent of valence $(m, n)$, and $\ell_{\alpha(p) \dot{\alpha}(q)}$ a conformal Killing tensor superfield of valence $(p, q)$, with $m \geq p$ and $n \geq q$. Then the following composite object

$$
\mathfrak{J}^{\alpha(m-p) \dot{\alpha}(n-q)}[\ell]:=J^{\alpha(m-p) \beta(p) \dot{\alpha}(n-q) \dot{\beta}(q)} \ell_{\beta(p) \dot{\beta}(q)}
$$

proves to be a conformal supercurrent of valence $(m-p, n-q)$.

\subsection{Conformal Killing spinor superfields and hypermultiplet}

A free superconformal hypermultiplet may be described by two primary superfields of dimension +1 , a chiral scalar $\phi$ and a complex linear scalar $\Gamma$,

$$
\left(\overline{\mathcal{D}}^{2}-4 R\right) \Gamma=0, \quad \mathbb{A} \Gamma=-\frac{2}{3} \Gamma
$$


The corresponding action

$$
S_{\text {hypermultiplet }}=\int \mathrm{d}^{4} x \mathrm{~d}^{2} \theta \mathrm{d}^{2} \bar{\theta} E\{\bar{\phi} \phi-\bar{\Gamma} \Gamma\},
$$

is super-Weyl invariant. ${ }^{8}$

A comment is required regarding the $\mathrm{U}(1)_{R}$ charge assignment in (4.20). In general, given a primary complex linear superfield $\Gamma$ of dimension $\Delta_{\Gamma}$ and $\mathrm{U}(1)_{R}$ charge $q_{\Gamma}$,

$$
\left(\overline{\mathcal{D}}^{2}-4 R\right) \Gamma=0, \quad \mathbb{A} \Gamma=q_{\Gamma} \Gamma,
$$

its charge and dimension are related to each other as

$$
q_{\Gamma}=\frac{2}{3} \Delta_{\Gamma}-\frac{4}{3},
$$

as a consequence of the identity

$$
\begin{aligned}
\delta_{\Sigma}\left(\overline{\mathcal{D}}^{2}-4 R\right)= & \Sigma\left(\overline{\mathcal{D}}^{2}-4 R\right)-4\left(\overline{\mathcal{D}}_{\dot{\alpha}} \Sigma\right) \overline{\mathcal{D}}^{\dot{\alpha}}+4\left(\overline{\mathcal{D}}^{\dot{\alpha}} \Sigma\right) \overline{\mathcal{D}}^{\dot{\beta}} \bar{M}_{\dot{\alpha} \dot{\beta}}-3\left(\overline{\mathcal{D}}_{\dot{\alpha}} \Sigma\right) \overline{\mathcal{D}}^{\dot{\alpha}} \mathbb{A} \\
& -\frac{3}{2}\left(\overline{\mathcal{D}}^{2} \Sigma\right) \mathbb{A}-2\left(\overline{\mathcal{D}}^{2} \Sigma\right)
\end{aligned}
$$

The above properties and relations are similar to those derived in [82] in the case of threedimensional $\mathcal{N}=2$ supergravity. The $\mathrm{U}(1)_{R}$ charge of $\Gamma$ was fixed in (4.20) in order for the action (4.21) to be super-Weyl invariant.

Given a conformal Killing spinor superfield $\ell_{\alpha}$ constrained according to (4.3),

$$
\mathcal{D}_{(\alpha} \ell_{\beta}=0, \quad \overline{\mathcal{D}}_{\dot{\alpha}} \ell_{\beta}=0,
$$

we associate with it the following transformation

$$
\begin{aligned}
& \delta \phi=\bar{\ell}_{\dot{\alpha}} \overline{\mathcal{D}}^{\dot{\alpha}} \Gamma+\frac{1}{2}\left(\overline{\mathcal{D}}_{\dot{\alpha}} \bar{\ell}^{\dot{\alpha}}\right) \Gamma, \\
& \delta \Gamma=-\ell^{\alpha} \mathcal{D}_{\alpha} \phi-\frac{1}{2}\left(\mathcal{D}^{\alpha} \ell_{\alpha}\right) \phi .
\end{aligned}
$$

It may be checked that $\overline{\mathcal{D}}_{\dot{\alpha}} \delta \phi=0$ and $\left(\overline{\mathcal{D}}^{2}-4 R\right) \delta \Gamma=0$. It may also be verified that $\delta \phi$ and $\delta \Gamma$ are primary superfields. A routine calculation shows that the hypermultiplet action (4.21) is invariant under the transformation (4.26), which is a curved superspace extension of that given in [79].

The massless hypermultiplet model (4.21) has a dual formulation realised in terms of two primary dimension- 1 chiral scalars $\phi$ and $\psi$. The dual action

$$
S_{\text {hypermultiplet }}^{(\text {dual }}=\int \mathrm{d}^{4} x \mathrm{~d}^{2} \theta \mathrm{d}^{2} \bar{\theta} E\{\bar{\phi} \phi+\bar{\psi} \psi\},
$$

\footnotetext{
${ }^{8} \mathrm{~A}$ chiral scalar $\phi$ and a complex linear scalar $\Gamma$ are the physical $\mathcal{N}=1$ superfields of the arctic hypermultiplet $[76,77]$ realised in $\mathcal{N}=1$ Minkowski superspace. In addition to $\phi$ and $\Gamma$, this off-shell hypermultiplet includes an infinite tail of auxiliary $\mathcal{N}=1$ superfields which are complex unconstrained and vanish on-shell. The superconformal arctic hypermultiplets were formulated in [78, 79]. General couplings of arctic hypermultiplets to $5 \mathrm{D} \mathcal{N}=1$ and $4 \mathrm{D} \mathcal{N}=2$ conformal supergravities were presented in [80, 81].
} 
is obviously super-Weyl invariant. In this dual formulation, the rigid symmetry (4.26) turns into

$$
\begin{aligned}
\delta \phi & =\frac{1}{2}\left(\overline{\mathcal{D}}^{2}-4 R\right)(\bar{\ell} \bar{\psi}), \\
\delta \psi & =-\frac{1}{2}\left(\overline{\mathcal{D}}^{2}-4 R\right)(\bar{\ell} \bar{\phi}) .
\end{aligned}
$$

Here $\bar{\ell}$ is the complex conjugate of a prepotential $\ell$ defined by

$$
\ell_{\alpha}=\mathcal{D}_{\alpha} \ell, \quad \mathbb{A} \ell=\frac{2}{3} \ell
$$

Equation (4.25) guarantees the existence of the prepotential $\ell$, which is is defined modulo arbitrary shifts

$$
\ell \rightarrow \ell+\bar{\lambda}, \quad \mathcal{D}_{\alpha} \bar{\lambda}=0
$$

The scalar $\ell$ is primary and of dimension -1 .

\subsection{Conformal Killing spinor superfields and nonlinear $\sigma$-models}

Now let us return to the nonlinear $\sigma$-model (3.33) and assume that its target space is a hyperkähler cone [83]. This means that (i) it is a hyperkähler manifold $\left(\mathcal{N}, g_{\mu \nu},\left(J_{A}\right)^{\mu}{ }_{\nu}\right)$, where $\mu, \nu=1, \ldots, 4 n$ and $A=1,2,3$; and (ii) it is a Kähler cone with respect to each complex structure. We pick one of the complex structures, say $J_{3}$, and introduce complex coordinates $\phi^{i}$ compatible with it. In these coordinates, $J_{3}$ has the form

$$
J_{3}=\left(\begin{array}{cc}
\mathrm{i} \delta^{i}{ }_{j} & 0 \\
0 & -\mathrm{i} \delta^{\bar{i}_{\bar{j}}}
\end{array}\right) .
$$

Two other complex structures, $J_{1}$ and $J_{2}$, become

$$
J_{1}=\left(\begin{array}{cc}
0 & g^{i \bar{k}} \bar{\omega}_{\bar{k} \bar{j}} \\
g^{\bar{i} k} \omega_{k j} & 0
\end{array}\right), \quad J_{2}=\left(\begin{array}{cc}
0 & \mathrm{i} g^{i \bar{k}} \bar{\omega}_{\bar{k} \bar{j}} \\
-\mathrm{i} g^{\bar{i} k} \omega_{k j} & 0
\end{array}\right)
$$

where $g_{i \bar{j}}(\phi, \bar{\phi})$ is the Kähler metric, and $\omega_{i j}(\phi)=-\omega_{j i}(\phi)$ is the holomorphic symplectic two-form.

It may be shown that the $\sigma$-model action (3.33) is invariant under the transformation

$$
\delta \phi^{i}=\frac{1}{2}\left(\overline{\mathcal{D}}^{2}-4 R\right)\left\{\bar{\ell} \omega^{i j} \chi_{j}\right\}
$$

The proof is analogous to that given in [84] in the case of Minkowski superspace. ${ }^{9}$ If we replace in the right-hand side of (4.33) $\bar{\ell} \rightarrow \bar{\ell}+\lambda$, with $\lambda$ chiral, then the $\lambda$-dependent part of the transformation a trivial symmetry (i.e., it vanishes on-shell) of the model.

\footnotetext{
${ }^{9}$ See also the seminal paper [85] for the non-superconformal case.
} 


\subsection{Symmetries of the massless Wess-Zumino operator}

Higher symmetries of relativistic wave equations have been studied over several decades. In particular, it was shown by Shapovalov and Shirokov [45] and, a decade later, by Eastwood [47] that the symmetry algebra of the d'Alembertian on $\mathbb{R}^{p, q}$, with $p+q \geq 3$, is isomorphic to (a quotient of) the universal enveloping algebra of the Lie algebra of conformal motions that span $\mathrm{SO}(p+1, q+1)$. Such infinite-dimensional algebras and their supersymmetric extensions play a fundamental role in higher-spin gauge theory [86]. Time has come to understand the higher symmetries of supersymmetric extensions of the d'Alembertian. To the best of our knowledge, so far there has appeared only one work on the topic, written by Howe and Lindström [58], where the symmetries of such operators are studied in flat superspaces in diverse dimensions.

In our discussion of superconformal field theories we re-derived the well-known result that conformal Killing supervector fields generate symmetries of the theories in question (3.26). In this section our analysis will be restricted to the free, massless theory obtained from (3.27) by setting $\lambda=0$,

$$
S[\phi, \bar{\phi}]=\int \mathrm{d}^{4} x \mathrm{~d}^{2} \theta \mathrm{d}^{2} \bar{\theta} E \bar{\phi} \phi .
$$

This model proves to have higher symmetries. The corresponding super-Weyl invariant equation of motion for $\bar{\phi}$ is

$$
\Pi \phi=0, \quad \Pi:=-\frac{1}{4}\left(\mathcal{D}^{2}-4 \bar{R}\right) .
$$

We will refer to $\Pi$ and its conjugate $\bar{\Pi}=-\frac{1}{4}\left(\overline{\mathcal{D}}^{2}-4 R\right.$ ) as the (massless) Wess-Zumino operators. These operators are examples of super-Laplacians discussed in [58].

Here we will study symmetries of the Wess-Zumino operator П. A scalar differential operator $\mathfrak{O}$ will be called a symmetry operator of $\Pi$ if it obeys the two conditions

$$
\begin{aligned}
\overline{\mathcal{D}}_{\dot{\alpha}} \mathfrak{O} \phi & =0, \\
\Pi \mathfrak{O} \phi & =0,
\end{aligned}
$$

for every on-shell chiral scalar $\phi,(4.35)$. Similar to the non-supersymmetric case [47], two symmetry operators $\mathfrak{O}$ and $\widetilde{\mathfrak{O}}$ are said to be equivalent, $\mathfrak{O} \sim \widetilde{\mathfrak{O}}$, if

$$
\widetilde{\mathfrak{O}}-\mathfrak{O}=\mathfrak{F}_{\dot{\alpha}} \overline{\mathcal{D}}^{\dot{\alpha}}+\mathfrak{H} \Pi \quad \Longleftrightarrow \mathfrak{O} \sim \widetilde{\mathfrak{O}},
$$

for some operators $\mathfrak{F}_{\dot{\alpha}}$ and $\mathfrak{H}$.

Since $\phi$ is a primary superfield of dimension +1 , we will impose one more condition on $\mathfrak{O}$, which is

$$
\delta_{\Sigma}(\mathfrak{O} \phi)=\Sigma \mathfrak{O} \phi
$$

In other words, we require $\mathfrak{O}$ to be a conformally invariant operator. In what follows, we will use bold-face capital letters, e.g. $\mathfrak{O}$, to denote symmetry operators which only satisfy conditions (4.36a) and (4.36b). 
Given a positive integer $n$, we look for an $n$ th-order symmetry operator

$$
\mathfrak{O}^{(n)}=\sum_{k=0}^{n} \zeta^{A_{1} \ldots A_{k}} \mathcal{D}_{A_{k}} \ldots \mathcal{D}_{A_{1}}
$$

where the coefficients may be chosen to be graded symmetric

$$
\zeta^{A_{1} \ldots A_{i} A_{i+1} \ldots A_{k}}=(-1)^{\varepsilon_{A_{i}} \varepsilon_{A_{i+1}}} \zeta^{A_{1} \ldots A_{i+1} A_{i} \ldots A_{k}}, \quad 1 \leq i \leq k-1 .
$$

Modulo the equivalence (4.37), $\mathfrak{O}^{(n)}$ may be brought to a canonical form given by

$$
\mathfrak{O}^{(n)}=\sum_{k=0}^{n} \zeta^{\alpha(k) \dot{\alpha}(k)} \mathcal{D}_{\alpha_{1} \dot{\alpha}_{1}} \ldots \mathcal{D}_{\alpha_{k} \dot{\alpha}_{k}}+\sum_{k=0}^{n-1} \zeta^{\alpha(k+1) \dot{\alpha}(k)} \mathcal{D}_{\alpha_{1} \dot{\alpha}_{1}} \ldots \mathcal{D}_{\alpha_{k} \dot{\alpha}_{k}} \mathcal{D}_{\alpha_{k+1}}
$$

Now, imposing the condition (4.36a) proves to lead to a number of constraints on the coefficients in (4.40), including the following

$$
\overline{\mathcal{D}}_{\dot{\beta}} \zeta^{\alpha(n) \dot{\alpha}(n)}=-2 \mathrm{i} \zeta^{\alpha(n)\left(\dot{\alpha}_{1} \ldots \dot{\alpha}_{n-1}\right.} \delta^{\left.\dot{\alpha}_{n}\right)} \dot{\beta},
$$

which is equivalent to

$$
\begin{aligned}
\overline{\mathcal{D}}_{\left(\dot{\alpha}_{1}\right.} \zeta_{\left.\alpha(n) \dot{\alpha}_{2} \ldots \dot{\alpha}_{n+1}\right)} & =0 \\
\zeta^{\alpha(n) \dot{\alpha}(n-1)} & =\frac{\mathrm{i} n}{2(n+1)} \overline{\mathcal{D}}_{\dot{\beta}} \zeta^{\alpha(n) \dot{\alpha}(n-1) \dot{\beta}} .
\end{aligned}
$$

We see that $\zeta_{\alpha(n) \dot{\alpha}(n-1)}$ is determined in terms of $\zeta_{\alpha(n) \dot{\alpha}(n)}$, and the latter is longitudinal linear. In fact, imposing the condition (4.36a) also leads to the equation

$$
\overline{\mathcal{D}}_{\dot{\beta}} \zeta^{\alpha(n) \dot{\alpha}(n-1)}=\mathrm{i} n \zeta^{\alpha(n)} \dot{\beta}(n-1) R,
$$

which automatically holds as a consequence of (4.42b).

Requiring the fulfilment of (4.38), a routine calculation allows us to express $\zeta_{\alpha(n-1) \dot{\alpha}(n-1)}$ in terms of the top component $\zeta_{\alpha(n) \dot{\alpha}(n)}$ as follows:

$$
\begin{aligned}
\zeta_{\alpha(n-1) \dot{\alpha}(n-1)}= & \frac{n^{2}}{2(n+1)} \mathcal{D}^{\beta \dot{\beta}} \zeta_{\beta \alpha(n-1) \dot{\beta} \dot{\alpha}(n-1)}-\frac{\mathrm{i} n^{2}}{4(n+1)(2 n+1)}\left[\mathcal{D}^{\beta}, \overline{\mathcal{D}}^{\dot{\beta}}\right] \zeta_{\beta \alpha(n-1) \dot{\beta} \dot{\alpha}(n-1)} \\
& +\frac{\mathrm{i} n(n+1)}{2(2 n+1)} G^{\beta \dot{\beta}} \zeta_{\beta \alpha(n-1) \dot{\beta} \dot{\alpha}(n-1)} .
\end{aligned}
$$

It should be remarked that the general solution to the constraint (4.42a) is

$$
\zeta_{\alpha(n) \dot{\alpha}(n)}=\overline{\mathcal{D}}_{\left(\dot{\alpha}_{1}\right.} v_{\left.\alpha(n) \dot{\alpha}_{2} \ldots \dot{\alpha}_{n}\right)}, \quad \mathbb{A} v_{\alpha(n) \dot{\alpha}(n-1)}=-v_{\alpha(n) \dot{\alpha}(n-1)},
$$

where the prepotential $v_{\alpha(n) \dot{\alpha}(n-1)}$ is defined modulo arbitrary shifts of the form

$$
v_{\alpha(n) \dot{\alpha}(n-1)} \rightarrow v_{\alpha(n) \dot{\alpha}(n-1)}+\tau_{\alpha(n) \dot{\alpha}(n-1)}, \quad \overline{\mathcal{D}}_{\left(\dot{\alpha}_{1}\right.} \tau_{\left.\alpha(n) \dot{\alpha}_{2} \ldots \dot{\alpha}_{n}\right)}=0
$$

Prepotential solution (4.45) will be important for our subsequent analysis. 
Suppose we have satisfied (4.36a). Then imposing the condition (4.36b) leads to new constraints on the coefficients in (4.40), including the following

$$
\mathcal{D}_{\left(\alpha_{1}\right.} \zeta_{\left.\alpha_{2} \ldots \alpha_{n+1}\right) \dot{\alpha}(n)}=0
$$

Equations (4.42a) and (4.47) tell us that the top component $\zeta_{\alpha(n) \dot{\alpha}(n)}$ in (4.40) obeys the same constraints (4.3) which are imposed on the conformal Killing tensor superfield $\ell_{\alpha(n) \dot{\alpha}(n)}$. These constraints are consistent with the reality condition $\bar{\zeta}_{\alpha(n) \dot{\alpha}(n)}=\zeta_{\alpha(n) \dot{\alpha}(n)}$, which will be assumed in what follows.

So far we have not attempted to find a general solution of the constrains (4.36) for $\mathfrak{O}^{(n)}$. Such a solution is easy to work out in the case of Minkowski superspace for which a consistent ansatz for an irreducible operator $\mathfrak{O}^{(n)}$ is given by

$$
\mathfrak{O}^{(n)}=\zeta^{\alpha(n) \dot{\alpha}(n)} \partial_{\alpha_{1} \dot{\alpha}_{1}} \ldots \partial_{\alpha_{n} \dot{\alpha}_{n}}+\zeta^{\alpha(n) \dot{\alpha}(n-1)} \partial_{\alpha_{1} \dot{\alpha}_{1}} \ldots \partial_{\alpha_{n-1} \dot{\alpha}_{n-1}} D_{\alpha_{n}}
$$

where $D_{A}=\left(\partial_{a}, D_{\alpha}, \bar{D}^{\dot{\alpha}}\right)$ are the flat superspace covariant derivatives. In this case the constraints (4.36) are equivalent to the relations

$$
\begin{aligned}
\bar{D}_{\left(\dot{\alpha}_{1}\right.} \zeta_{\left.\alpha(n) \dot{\alpha}_{2} \ldots \dot{\alpha}_{n+1}\right)} & =0, \quad D_{\left(\alpha_{1} \zeta_{\left.\alpha_{2} \ldots \alpha_{n+1}\right) \dot{\alpha}(n)}=0,\right.} \\
\zeta_{\alpha(n) \dot{\alpha}(n-1)} & =-\frac{\mathrm{i} n}{2(n+1)} \bar{D}^{\dot{\beta}} \zeta_{\alpha(n) \dot{\alpha}(n-1) \dot{\beta}} .
\end{aligned}
$$

We emphasise that (4.48) is a flat-superspace solution of the constrains (4.36). If the equation (4.38) is also required, then certain lower-order terms must be added to (4.48), as follows from from eq. (4.44) and also from the explicit expressions for $\mathfrak{O}^{(1)}$ and $\mathfrak{O}^{(2)}$ given below.

The explicit structure of the flat-superspace symmetry (4.48) tells us that it is always possible to construct a solution for the coefficients $\zeta^{\alpha(k) \dot{\alpha}(k)}$ and $\zeta^{\alpha(k+1) \dot{\alpha}(k)}$ of all operators in (4.40) of order $n-1, \ldots, 0$ which are proportional to certain components of the torsion tensor and their covariant derivatives.

The above consideration can be extended to anti-de Sitter superspace, $\operatorname{AdS}^{4 \mid 4}$, which is characterised by the following algebra of covariant derivatives [3]

$$
\begin{aligned}
\left\{\mathcal{D}_{\alpha}, \overline{\mathcal{D}}_{\dot{\alpha}}\right\} & =-2 \mathrm{i} \mathcal{D}_{\alpha \dot{\alpha}}, \\
\left\{\mathcal{D}_{\alpha}, \mathcal{D}_{\beta}\right\} & =-4 \bar{\mu} M_{\alpha \beta}, \\
{\left[\mathcal{D}_{\alpha}, \mathcal{D}_{\beta \dot{\beta}}\right] } & =\mathrm{i} \bar{\mu} \varepsilon_{\alpha \beta} \overline{\mathcal{D}}_{\dot{\beta}}, \\
{\left[\mathcal{D}_{\alpha \dot{\alpha}}, \mathcal{D}_{\beta \dot{\beta}}\right] } & =-2 \bar{\mu} \mu\left(\varepsilon_{\alpha \beta} \bar{M}_{\dot{\alpha} \dot{\beta}}+\varepsilon_{\dot{\alpha} \dot{\beta}} M_{\alpha \beta}\right),
\end{aligned}
$$

with $\mu \neq 0$ being a complex parameter (the scalar curvature of $\mathrm{AdS}_{4}$ is equal to $-12|\mu|^{2}$ ). One can show that the following irreducible operator is a consistent ansatz for $\mathfrak{O}^{(n)}$

$$
\mathfrak{O}^{(n)}=\zeta^{\alpha(n) \dot{\alpha}(n)} \mathcal{D}_{\alpha_{1} \dot{\alpha}_{1}} \ldots \mathcal{D}_{\alpha_{n} \dot{\alpha}_{n}}+\zeta^{\alpha(n) \dot{\alpha}(n-1)} \mathcal{D}_{\alpha_{1} \dot{\alpha}_{1}} \ldots \mathcal{D}_{\alpha_{n-1} \dot{\alpha}_{n-1}} \mathcal{D}_{\alpha_{n}}
$$


Here, the constraints (4.36) are equivalent to

$$
\begin{aligned}
\overline{\mathcal{D}}_{\left(\dot{\alpha}_{1}\right.} \zeta_{\left.\alpha(n) \dot{\alpha}_{2} \ldots \dot{\alpha}_{n+1}\right)} & =0, \quad \mathcal{D}_{\left(\alpha_{1}\right.} \zeta_{\left.\alpha_{2} \ldots \alpha_{n+1}\right) \dot{\alpha}(n)}=0, \\
\zeta_{\alpha(n) \dot{\alpha}(n-1)} & =-\frac{\mathrm{i} n}{2(n+1)} \overline{\mathcal{D}}^{\dot{\beta}} \zeta_{\alpha(n) \dot{\alpha}(n-1) \dot{\beta}}, \\
\overline{\mathcal{D}}_{\dot{\beta}} \zeta_{\alpha(n) \alpha(n-1)} & =\mathrm{i} n \mu \zeta_{\alpha(n) \dot{\alpha}(n-1) \dot{\beta}} .
\end{aligned}
$$

We again emphasise that (4.51) is an AdS-superspace solution of the constraints (4.36).

We now determine $\mathfrak{O}^{(1)}$ and $\mathfrak{O}^{(2)}$ in U(1) superspace. Setting $n=1$ in (4.40) gives

$$
\mathfrak{D}^{(1)} \phi=\left(\zeta^{\alpha \dot{\alpha}} \mathcal{D}_{\alpha \dot{\alpha}}+\zeta^{\alpha} \mathcal{D}_{\alpha}+\zeta\right) \phi .
$$

Requiring $\mathfrak{O}^{(1)} \phi$ to be chiral allows us to obtain

$$
\overline{\mathcal{D}}_{\dot{\alpha}} \zeta=-\frac{\mathrm{i}}{3} \zeta_{\dot{\alpha}}^{\alpha} X_{\alpha} .
$$

Additionally, the property that the transformed field remains primary with dimension +1 leads to the following super-Weyl transformation laws for the parameters

$$
\begin{aligned}
\delta_{\Sigma} \zeta_{\alpha \dot{\alpha}} & =-\Sigma \zeta_{\alpha \dot{\alpha}} \\
\delta_{\Sigma} \zeta_{\alpha} & =-\frac{\Sigma}{2} \zeta_{\alpha}-\mathrm{i} \overline{\mathcal{D}}^{\dot{\alpha}} \Sigma \zeta_{\alpha \dot{\alpha}}, \\
\delta_{\Sigma} \zeta & =-2 \mathcal{D}^{\alpha} \Sigma \zeta_{\alpha}-\mathcal{D}^{\alpha \dot{\alpha}} \Sigma \zeta_{\alpha \dot{\alpha}}+\frac{\mathrm{i}}{2}\left[\mathcal{D}^{\alpha}, \overline{\mathcal{D}}^{\dot{\alpha}}\right] \Sigma \zeta_{\alpha \dot{\alpha}} .
\end{aligned}
$$

A solution to (4.53) which is consistent with (4.54c) is given by

$$
\zeta=-\frac{\mathrm{i}}{3} v^{\alpha} X_{\alpha}+\frac{\mathrm{i}}{12}\left(\overline{\mathcal{D}}^{2}-4 R\right) \mathcal{D}^{\alpha} v_{\alpha},
$$

with the prepotential $v_{\alpha}$ being defined according to (4.45).

It should be emphasised that the second (chiral) term in (4.55) is not determined by the condition (4.36a) which only constrains $\zeta$ to satisfy (4.53). However, this term is uniquely fixed if we further require the condition (4.38) to hold. Making use of the identity

$$
\begin{aligned}
{\left[\overline{\mathcal{D}}^{2}, \mathcal{D}_{\alpha}\right]=} & -4\left(G_{\alpha \dot{\alpha}}-\mathrm{i} \mathcal{D}_{\alpha \dot{\alpha}}\right) \overline{\mathcal{D}}^{\dot{\alpha}}+4 R \mathcal{D}_{\alpha}-4 \overline{\mathcal{D}}^{\dot{\alpha}} G_{\alpha}{ }^{\dot{\beta}} \bar{M}_{\dot{\alpha} \dot{\beta}}+8 W_{\alpha}{ }^{\beta \gamma} M_{\beta \gamma} \\
& -\frac{4}{3} X^{\beta} M_{\alpha \beta}-2 X_{\alpha} \mathbb{A},
\end{aligned}
$$

one may obtain from (4.55) a different expression for $\zeta$ given by

$$
\zeta=\left(\frac{1}{4} \mathcal{D}_{\alpha \dot{\alpha}}+\frac{\mathrm{i}}{3} G_{\alpha \dot{\alpha}}-\frac{\mathrm{i}}{24}\left[\mathcal{D}_{\alpha}, \overline{\mathcal{D}}_{\dot{\alpha}}\right]\right) \zeta^{\alpha \dot{\alpha}},
$$

which reveals that all parameters of the operator $\mathfrak{O}^{(1)}$ are expressible in terms of the vector $\zeta^{\alpha \dot{\alpha}}$. This is in agreement with the results of the top-down approach (3.3).

Once the background superspace $\left(\mathcal{M}^{4 \mid 4}, \mathcal{D}\right)$ possesses first-order symmetry operators $\mathfrak{O}_{\zeta_{1}}^{(1)}, \ldots, \mathfrak{O}_{\zeta_{n}}^{(1)}$, , we can generate a higher-order symmetry operator, $\widetilde{\mathfrak{O}}^{(n)}$, defined by

$$
\widetilde{\mathfrak{D}}^{(n)}:=\mathfrak{O}_{\zeta_{1}}^{(1)} \ldots \mathfrak{O}_{\zeta_{n}}^{(1)}, \quad n=2,3, \ldots
$$


By construction, it satisfies the conditions (4.36) and (4.38). Of course, it does not have the canonical form (4.40), however it may be brought to such a form by factoring out a contribution of the type (4.37).

Next, we consider the $n=2$ case

$$
\mathfrak{D}^{(2)} \phi=\left(\zeta^{\alpha \beta \dot{\alpha} \dot{\beta}} \mathcal{D}_{\alpha \dot{\alpha}} \mathcal{D}_{\beta \dot{\beta}}+\zeta^{\alpha \beta \dot{\alpha}} \mathcal{D}_{\alpha \dot{\alpha}} \mathcal{D}_{\beta}+\zeta^{\alpha \dot{\alpha}} \mathcal{D}_{\alpha \dot{\alpha}}+\zeta^{\alpha} \mathcal{D}_{\alpha}+\zeta\right) \phi
$$

Requiring the conditions (4.36) leads to the integrability conditions

$$
\begin{aligned}
\overline{\mathcal{D}}_{\dot{\alpha}} \zeta_{\beta \dot{\beta}}= & 2 \mathrm{i} \varepsilon_{\dot{\alpha} \dot{\beta}} \zeta_{\beta}+2 \mathrm{i} \zeta^{\alpha \gamma}{ }_{\alpha \dot{\beta}} W_{\alpha \beta \gamma}-\mathrm{i} \zeta_{\beta \dot{\alpha} \dot{\beta}}^{\alpha}\left(\mathcal{D}_{\alpha} R+\frac{1}{3} X_{\alpha}\right)-\mathrm{i} \zeta_{\beta}{ }_{\dot{\alpha}} \dot{\gamma}^{\dot{\mathcal{D}}} \bar{\gamma}_{\dot{\gamma}} G_{\gamma \dot{\beta}} \\
& -\zeta_{\beta(\dot{\alpha}}^{\alpha} G_{\alpha \dot{\beta})}, \\
\overline{\mathcal{D}}_{\dot{\alpha}} \zeta_{\alpha}= & \mathrm{i} \zeta_{\alpha \dot{\alpha}} R+\frac{\mathrm{i}}{2} \zeta_{\alpha}{ }^{\beta} \dot{\alpha}\left(\mathcal{D}_{\beta} R+\frac{1}{3} X_{\beta}\right)+\mathrm{i} \zeta_{\alpha}{ }^{\beta} \dot{\alpha}{ }^{\dot{\beta}} \mathcal{D}_{\beta \dot{\beta}} R-\mathrm{i} \zeta_{\dot{\alpha}}^{\beta \gamma} W_{\alpha \beta \gamma} \\
& -\zeta_{\alpha}{ }^{\beta} \dot{\alpha}{ }^{\dot{\beta}} G_{\beta \dot{\beta}}, \\
\overline{\mathcal{D}}_{\dot{\alpha}} \zeta= & \frac{\mathrm{i}}{3} \zeta^{\alpha}{ }_{\dot{\alpha}} X_{\alpha}+\frac{\mathrm{i}}{3} \zeta^{\alpha \beta}{ }_{\dot{\alpha}} \mathcal{D}_{\alpha} X_{\beta}+\frac{1}{3} \zeta^{\alpha \beta} \dot{\alpha}^{\dot{\beta}} X_{\alpha} G_{\beta \dot{\beta}}-\frac{\mathrm{i}}{3} \zeta_{\dot{\alpha}}^{\alpha \beta} \mathcal{D}_{\beta \dot{\beta}} X_{\alpha} .
\end{aligned}
$$

So far we have not taken into account the condition (4.38); the transformed field, $\mathfrak{O}^{(2)} \phi$, retains the property of being primary and of dimension +1 . This condition fixes the super-Weyl transformation laws for the parameters

$$
\begin{aligned}
\delta_{\Sigma} \zeta_{\alpha \beta \dot{\alpha} \dot{\beta}}= & -2 \Sigma \zeta_{\alpha \beta \dot{\alpha} \dot{\beta}}, \\
\delta_{\Sigma} \zeta_{\alpha \beta \dot{\alpha}}= & -\frac{3}{2} \Sigma \zeta_{\alpha \beta \dot{\alpha}}+2 \mathrm{i} \overline{\mathcal{D}}^{\dot{\beta}} \Sigma \zeta_{\alpha \beta \dot{\alpha} \dot{\beta}}, \\
\delta_{\Sigma} \zeta_{\alpha \dot{\alpha}}= & -\Sigma \zeta_{\alpha \dot{\alpha}}-4 \mathcal{D}^{\beta \dot{\beta}} \Sigma \zeta_{\alpha \beta \dot{\alpha} \dot{\beta}}+\mathrm{i}\left[\mathcal{D}^{\beta}, \overline{\mathcal{D}}^{\beta}\right] \Sigma \zeta_{\alpha \beta \dot{\alpha} \dot{\beta}}-4 \mathcal{D}^{\beta} \Sigma \zeta_{\alpha \beta \dot{\alpha}}, \\
\delta_{\Sigma} \zeta_{\alpha}= & -\frac{1}{2} \Sigma \zeta_{\alpha}+\mathrm{i} \overline{\mathcal{D}}^{\dot{\alpha}} \mathcal{D}^{\beta \dot{\beta}} \Sigma \zeta_{\alpha \beta \dot{\alpha} \dot{\beta}}-2 \mathcal{D}^{\beta \dot{\beta}} \Sigma \zeta_{\alpha \beta \dot{\alpha} \dot{\beta}}+\mathrm{i} \mathcal{D}^{\dot{\alpha}} \Sigma \zeta_{\alpha \dot{\alpha}}, \\
\delta_{\Sigma} \zeta= & \frac{\mathrm{i}}{2} \mathcal{D}^{\alpha \dot{\alpha}}\left[\mathcal{D}^{\beta}, \overline{\mathcal{D}}^{\dot{\beta}}\right] \Sigma \zeta_{\alpha \beta \dot{\alpha} \dot{\beta}}-2 \mathcal{D}^{\alpha} \mathcal{D}^{\beta \dot{\beta}} \Sigma \zeta_{\alpha \beta \dot{\beta}}+\frac{\mathrm{i}}{2}\left[\mathcal{D}^{\alpha}, \overline{\mathcal{D}}^{\dot{\alpha}}\right] \Sigma \zeta_{\alpha \dot{\alpha}} \\
& -2 \mathcal{D}^{\alpha} \Sigma \zeta_{\alpha}-\mathcal{D}^{\alpha \dot{\alpha}} \Sigma \zeta_{\alpha \dot{\alpha}}-\mathcal{D}^{\alpha \dot{\alpha}} \mathcal{D}^{\beta \dot{\beta}} \Sigma \zeta_{\alpha \beta \dot{\alpha} \dot{\beta}} .
\end{aligned}
$$

The requirement that (4.36) and (4.38) are satisfied leads to the unique solution

$$
\begin{aligned}
\zeta_{\alpha \dot{\alpha}}= & \frac{2}{3} \mathcal{D}^{\beta \dot{\beta}} \zeta_{\alpha \beta \dot{\alpha} \dot{\beta}}-\frac{\mathrm{i}}{15}\left[\mathcal{D}^{\beta}, \overline{\mathcal{D}}^{\dot{\beta}}\right] \zeta_{\alpha \beta \dot{\alpha} \dot{\beta}}+\frac{3 \mathrm{i}}{5} \zeta_{\alpha \beta \dot{\alpha} \dot{\beta}} G^{\beta \dot{\beta}} \\
\zeta_{\alpha}= & -\frac{2 \mathrm{i}}{15} \overline{\mathcal{D}}^{\dot{\alpha}} \mathcal{D}^{\beta \dot{\beta}} \zeta_{\alpha \beta \dot{\alpha} \dot{\beta}}-\frac{1}{10} \zeta_{\alpha \beta \dot{\alpha} \dot{\beta}} \overline{\mathcal{D}}^{\dot{\alpha}} G^{\beta \dot{\beta}}+\frac{1}{15} \overline{\mathcal{D}}^{\dot{\alpha}} \zeta_{\alpha \beta \dot{\alpha} \dot{\beta}} G^{\beta \dot{\beta}}, \\
\zeta= & \frac{1}{15} \mathcal{D}^{\alpha \dot{\alpha}} \mathcal{D}^{\beta \dot{\beta}} \zeta_{\alpha \beta \dot{\alpha} \dot{\beta}}-\frac{\mathrm{i}}{60} \mathcal{D}^{\alpha \dot{\alpha}}\left[\mathcal{D}^{\beta}, \overline{\mathcal{D}}^{\dot{\beta}}\right] \zeta_{\alpha \beta \dot{\alpha} \dot{\beta}}+\frac{7 \mathrm{i}}{30} \mathcal{D}^{\alpha \dot{\alpha}} \zeta_{\alpha \beta \dot{\alpha} \dot{\beta}} G^{\beta \dot{\beta}} \\
& +\frac{1}{30}\left[\mathcal{D}^{\alpha}, \overline{\mathcal{D}}^{\dot{\alpha}}\right] \zeta_{\alpha \beta \dot{\alpha} \dot{\beta}} G^{\beta \dot{\beta}}+\frac{1}{20} \mathcal{D}^{\alpha} \zeta_{\alpha \beta \dot{\alpha} \dot{\beta}} \overline{\mathcal{D}}^{\dot{\alpha}} G^{\beta \dot{\beta}}-\frac{13}{60} \overline{\mathcal{D}}^{\dot{\alpha}} \zeta_{\alpha \beta \dot{\alpha} \dot{\beta}} \mathcal{D}^{\alpha} G^{\beta \dot{\beta}} \\
& +\frac{2 \mathrm{i}}{5} \zeta_{\alpha \beta \dot{\alpha} \dot{\beta}} \mathcal{D}^{\alpha \dot{\alpha}} G^{\beta \dot{\beta}}+\frac{3}{20} \zeta_{\alpha \beta \dot{\alpha} \dot{\beta}}\left[\mathcal{D}^{\dot{\alpha}}, \overline{\mathcal{D}}^{\dot{\alpha}}\right] G^{\beta \dot{\beta}}-\frac{1}{5} \zeta_{\alpha \beta \dot{\alpha} \dot{\beta}} G^{\alpha \dot{\alpha}} G^{\beta \dot{\beta}} .
\end{aligned}
$$

Thus, this transformation is completely determined by the conformal Killing tensor $\zeta^{\alpha \beta \dot{\alpha} \dot{\beta}}$. It is crucial to note that if we relax condition (4.38), the solution ceases to be uniquely 
defined and may be constructed in such a way that the coefficients $\zeta_{\alpha \dot{\alpha}}, \zeta_{\alpha}$ and $\zeta$ vanish in the flat (or AdS) superspace limit.

In the case of a symmetry operator (4.40) of arbitrary order, we expect that our conceptual results for $\mathfrak{O}^{(1)}$ and $\mathfrak{O}^{(2)}$ generalise; all components are uniquely determined in terms of $\zeta_{\alpha(n) \dot{\alpha}(n)}$ and a suitable flat (or AdS) superspace limit may be constructed.

\subsection{Supersymmetric even Schouten-Nijenhuis bracket}

In analogy with the space of conformal Killing supervector fields, we wish to endow our construction with an additional structure allowing us to combine two conformal Killing tensors and produce a third. Consider two such tensors $\zeta_{\alpha(m) \dot{\alpha}(m)}^{1}$ and $\zeta_{\alpha(n) \dot{\alpha}(n)}^{2}$. It can then be shown that the following bracket (an implicit symmetrisation over all $\alpha$-indices and, independently, all $\dot{\alpha}$-indices is assumed below)

$$
\begin{aligned}
& {\left[\zeta^{1}, \zeta^{2}\right]_{\alpha(m+n-1) \dot{\alpha}(m+n-1)}=} \\
& -\frac{m}{2} \zeta_{\alpha(m-1)}^{1}{ }^{\beta} \dot{\alpha}(m-1){ }^{\dot{\beta}} \mathcal{D}_{\beta \dot{\beta}} \zeta_{\alpha(n) \dot{\alpha}(n)}^{2}+\frac{n}{2} \zeta_{\alpha(n-1)}^{2} \dot{\alpha}(n-1){ }^{\dot{\beta}} \mathcal{D}_{\beta \dot{\beta}} \zeta_{\alpha(m) \dot{\alpha}(m)}^{1} \\
& -\frac{\mathrm{i} m n}{4(m+1)(n+1)}\left(\overline{\mathcal{D}}_{\dot{\beta}} \zeta_{\alpha(m)}^{1}{ }_{\dot{\beta}(m-1)}^{\dot{\beta}} \mathcal{D}_{\beta} \zeta_{\alpha(n-1)}^{2}{ }_{\dot{\alpha}(n)}-\overline{\mathcal{D}}_{\dot{\beta}} \zeta_{\alpha(n)}^{2} \dot{\beta} \dot{\alpha}(n-1) \mathcal{D}_{\beta} \zeta_{\alpha(m-1)}^{1}{ }_{\dot{\alpha}(m)}\right) \\
& +\frac{\mathrm{i} m n}{2}\left(\zeta_{\alpha(m) \dot{\alpha}(m-1)}^{1} \dot{\beta}_{\alpha(n-1)}^{2}{ }_{\dot{\alpha}(n-1)}-\zeta_{\alpha(n) \dot{\alpha}(n-1)}^{2} \dot{\beta}_{\alpha(m-1)}^{1}{ }^{\beta} \dot{\alpha}(m-1)\right) G_{\beta \dot{\beta}}
\end{aligned}
$$

also satisfies these conditions and hence is a new conformal Killing tensor superfield. Hence, for a given supergravity background, the set of conformal Killing tensor superfields $\zeta_{\alpha(n) \dot{\alpha}(n)}$ is a superalgebra with respect to the above bracket.

The $G_{\beta \dot{\beta}}$-dependent terms in (4.63) can be removed by redefining the vector covariant derivative by the rule

$$
\mathcal{D}_{\alpha \dot{\alpha}} \rightarrow \widetilde{\mathcal{D}}_{\alpha \dot{\alpha}}=\mathcal{D}_{\alpha \dot{\alpha}}+\frac{\mathrm{i}}{2}\left(G_{\dot{\alpha}}^{\beta} M_{\alpha \beta}-G_{\alpha}{ }^{\dot{\beta}} \bar{M}_{\dot{\alpha} \dot{\beta}}\right)
$$

or, equivalently,

$$
\mathcal{D}_{a} \rightarrow \widetilde{\mathcal{D}}_{a}+\frac{1}{4} \varepsilon_{a b c d} G^{b} M^{c d}
$$

The specific feature of the covariant derivatives $\widetilde{\mathcal{D}}_{A}=\left(\widetilde{\mathcal{D}}_{a}, \mathcal{D}_{\alpha}, \overline{\mathcal{D}}^{\dot{\alpha}}\right)$ is the torsion-free condition $\widetilde{T}_{a b}{ }^{c}=0$. In terms of the covariant derivatives $\widetilde{\mathcal{D}}_{A}$, the bracket (4.63) coincides with the one proposed in [57] where it was called the "supersymmetric even SchoutenNijenhuis bracket."

In the case of $\mathcal{N}=1$ AdS superspace, the bracket (4.63) coincides with the one given in [49] for Killing tensor superfields.

\section{Isometries of curved superspace}

As is well known, every off-shell formulation for $\mathcal{N}=1$ supergravity is obtained by coupling conformal supergravity to a compensating supermultiplet. Different supergravity theories correspond to different compensators, see, e.g., [37, 87-89]. For a given theory, 
the compensator $\Xi$ is a nowhere vanishing primary scalar superfield, which obeys certain constraints and has a non-zero dimension, $\Delta_{\Xi} \neq 0$, and some $\mathrm{U}(1)_{R}$ charge $q_{\Xi}$. In the case of new minimal supergravity, $q_{\Xi}=0$ and the compensator is real. For the old minimal and non-minimal formulations, $q_{\Xi}$ is non-zero. Once $\Xi$ is specified, supergravity background is a triple $\left(\mathcal{M}^{4 \mid 4}, \mathcal{D}, \Xi\right)$.

\subsection{Off-shell supergravity and Killing vector superfields}

Let $\xi=\xi^{B} E_{B}$ be a conformal Killing supervector field on $\left(\mathcal{M}^{4 \mid 4}, \mathcal{D}\right)$,

$$
\left(\delta_{\mathcal{K}[\xi]}+\delta_{\Sigma[\xi]}\right) \mathcal{D}_{A}=0
$$

It is called a Killing supervector field if it leaves the compensator $\Xi$ invariant,

$$
\left(\delta_{\mathcal{K}[\xi]}+\Delta_{\Xi} \Sigma[\xi]\right) \Xi=0
$$

The latter condition can be rewritten in the form

$$
\xi^{B} \mathcal{D}_{B} \Xi+\left(\Delta_{\Xi} \Sigma[\xi]+\mathrm{i} q_{\Xi} \rho[\xi]\right) \Xi=0 .
$$

The set of all Killing supervector fields on $\left(\mathcal{M}^{4 \mid 4}, \mathcal{D}, \Xi\right)$ is a Lie superalgebra.

The Killing equations (5.1) are super-Weyl invariant in the sense that they hold for all conformally related supergravity backgrounds. In the presence of a compensator, the notion of conformally related superspaces given in section 3.3 should be generalised as follows. Two supergravity backgrounds $\left(\mathcal{M}^{4 \mid 4}, \hat{\mathcal{D}}, \hat{\Xi}\right)$ and $\left(\mathcal{M}^{4 \mid 4}, \mathcal{D}, \Xi\right)$ are said to be conformally related provided the covariant derivatives $\hat{\mathcal{D}}_{A}$ and $\mathcal{D}_{A}$ are related to each other according to (3.15), and the same super-Weyl parameter $\Sigma$ relates the compensators,

$$
\hat{\Xi}=\mathrm{e}^{\Delta_{\Xi} \Sigma} \Xi \text {. }
$$

Applying a super-Weyl transformation allows us to choose the gauge

$$
\Xi \Xi=1
$$

and then (5.2) reduces to

$$
\Sigma[\xi]=0 \quad \Longleftrightarrow \quad \mathcal{D}^{\alpha} \xi_{\alpha}+\overline{\mathcal{D}}_{\dot{\alpha}} \bar{\xi}^{\dot{\alpha}}=0 \quad \Longrightarrow \quad \mathcal{D}_{a} \xi^{a}=0 .
$$

In this gauge the Killing equations (5.1) take the simplified form

$$
\delta_{\mathcal{K}[\xi]} \mathcal{D}_{A}=\left[\mathcal{K}[\xi], \mathcal{D}_{A}\right]=0 .
$$

Once $\Sigma[\xi]=0$ the left-hand side of each relation in (3.14) is equal to zero, and therefore the right-hand side must vanish as well. It is an instructive exercise to demonstrate, with the aid of the relations (3.4) and (3.8), that this is indeed the case.

For $q_{\Xi} \neq 0$ it is always possible to impose a stronger gauge condition than (5.4). Indeed, applying a combined super-Weyl and local $\mathrm{U}(1)_{R}$ transformation allows us to set

$$
\Xi=1,
$$


and then the Killing condition (5.2) turns into

$$
\Sigma[\xi]=0, \quad \xi^{B} \Phi_{B}+\rho[\xi]=0 .
$$

When studying the symmetries of bosonic supergravity backgrounds, we will keep some of the components of $\Xi$ alive and, instead, impose the so-called Weyl multiplet gauge described in appendix C.

\subsection{Conformal compensators}

In this subsection we briefly review the structure of the compensating supermultiplets which correspond to the old minimal $[6,12,13]$ and new minimal $[38,39]$ formulations for $\mathcal{N}=1$ supergravity. The non-minimal formulations for Poincaré [87, 90, 91] and AdS supergravity [60] will not be discussed here.

In the old minimal formulation, the compensator is a nowhere vanishing primary chiral scalar $S_{0}$ with the superconformal properties

$$
\overline{\mathcal{D}}_{\dot{\alpha}} S_{0}=0, \quad \Delta_{S_{0}}=1, \quad q_{S_{0}}=\frac{2}{3} .
$$

The supergravity action is

$$
S_{\mathrm{SG}, \text { old }}=-\frac{3}{\kappa^{2}} \int \mathrm{d}^{4} x \mathrm{~d}^{2} \theta \mathrm{d}^{2} \bar{\theta} E \bar{S}_{0} S_{0}+\left\{\frac{\mu}{\kappa^{2}} \int \mathrm{d}^{4} x \mathrm{~d}^{2} \theta \mathcal{E} S_{0}^{3}+\text { c.c. }\right\},
$$

where $\kappa$ is the gravitational coupling constant, and $\mu$ is a complex parameter related to the cosmological constant. Making use of the super-Weyl and local $\mathrm{U}(1)_{R}$ transformations, the chiral compensator can be gauged away resulting with

$$
S_{0}=1 \quad \Longrightarrow \quad \Phi_{A}=0 \quad \Longrightarrow \quad X_{\alpha}=0
$$

In the new minimal formulation, the compensator is a nowhere vanishing primary scalar $L$ constrained by ${ }^{10}$

$$
\bar{L}=L, \quad\left(\overline{\mathcal{D}}^{2}-4 R\right) L=0 \quad \Longrightarrow \quad \Delta_{L}=2 .
$$

The supergravity action is

$$
S_{\mathrm{SG}, \text { new }}=\frac{3}{\kappa^{2}} \int \mathrm{d}^{4} x \mathrm{~d}^{2} \theta \mathrm{d}^{2} \bar{\theta} E L \ln \frac{L}{\bar{S}_{0} S_{0}},
$$

where the chiral scalar $S_{0}$, eq. (5.9) is a pure gauge degree of freedom. The super-Weyl invariance allows one to choose the gauge

$$
L=1 \quad \Longrightarrow \quad R=0 \text {. }
$$

\footnotetext{
${ }^{10}$ The linear compensator (5.12) was introduced in [88]. It is a tensor multiplet [92] such that its field strength $L$ is nowhere vanishing.
} 


\subsection{Killing spinor superfields and massive hypermultiplet}

To describe a massive hypermultiplet, we consider the following generalisation of (4.27)

$$
S_{\text {hypermultiplet }}^{(m)}=\int \mathrm{d}^{4} x \mathrm{~d}^{2} \theta \mathrm{d}^{2} \bar{\theta} E\{\bar{\phi} \phi+\bar{\psi} \psi\}+\left\{m \mathrm{i} \int \mathrm{d}^{4} x \mathrm{~d}^{2} \theta \mathcal{E} S_{0} \psi \phi+\text { c.c. }\right\},
$$

where $m$ is a real mass parameter. When analysing this model, we will adopt the superWeyl gauge $S_{0}=1$, and therefore the $\mathrm{U}(1)_{R}$ connection is equal to zero, $\Phi_{A}=0$.

Through a direct computation, we find that the transformation (4.28) is also a symmetry of the massive theory only if $\ell$ is constrained to be real,

$$
\bar{\ell}=\ell \quad \Longrightarrow \quad \mathcal{D}_{\alpha \dot{\alpha}} \ell=0,
$$

where we have used the relations (4.25) and (4.29), which imply

$$
\overline{\mathcal{D}}_{\dot{\alpha}} \mathcal{D}_{\alpha} \ell=0
$$

These conditions may be shown to have the following non-trivial implication:

$$
\mathcal{D}_{\alpha}\left(\overline{\mathcal{D}}^{2}-4 R\right) \ell=-4 \overline{\mathcal{D}}^{\dot{\alpha}}\left(G_{\alpha \dot{\alpha}} \ell\right)
$$

Now, in conjunction with the identity $\overline{\mathcal{D}}_{\dot{\alpha}}\left(\overline{\mathcal{D}}^{2}-4 R\right) \ell=0$, we observe that

$$
G_{\alpha \dot{\alpha}}=0 \quad \Longrightarrow \quad\left(\overline{\mathcal{D}}^{2}-4 R\right) \ell=\text { const } .
$$

The condition $G_{\alpha \dot{\alpha}}=0$ means that the background under consideration is Einstein, i.e. it is a solution of supergravity equations of motion.

To realise a second supersymmetry transformation in $\mathcal{N}=1$ AdS superspace, refs. $[49,93]$ made use of a background scalar superfield $\varepsilon$ subject to the constraints

$$
\bar{\varepsilon}=\varepsilon, \quad \overline{\mathcal{D}}_{\dot{\alpha}} \mathcal{D}_{\alpha} \varepsilon=0, \quad\left(\overline{\mathcal{D}}^{2}-4 \mu\right) \varepsilon=0 .
$$

The parameter $\varepsilon$ naturally originates within the $\mathcal{N}=2$ AdS superspace approach [28]. The Killing superfield $\ell$ introduced above contains two additional scalar parameters as compared with $\varepsilon$.

\subsection{Symmetries of the massive Wess-Zumino operator}

A massive scalar supermultiplet in curved superspace is described by the action

$$
S[\phi, \bar{\phi}]=\int \mathrm{d}^{4} x \mathrm{~d}^{2} \theta \mathrm{d}^{2} \bar{\theta} E \bar{\phi} \phi+\left\{\frac{m}{2} \int \mathrm{d}^{4} x \mathrm{~d}^{2} \theta \mathcal{E} S_{0} \phi^{2}+\text { c.c. }\right\}, \quad \overline{\mathcal{D}}_{\dot{\alpha}} \phi=0,
$$

with $m=\bar{m}$ a mass parameter. In what follows we will work in the super-Weyl gauge $S_{0}=1$. Then the equations of motion are

$$
\mathcal{H}_{m}\left(\begin{array}{c}
\phi \\
\bar{\phi}
\end{array}\right)=0, \quad \mathcal{H}_{m}=\left(\begin{array}{cc}
m & \bar{\Pi} \\
\Pi & m
\end{array}\right), \quad \Pi:=-\frac{1}{4}\left(\mathcal{D}^{2}-4 \bar{R}\right) .
$$


We now wish to understand what additional conditions must be imposed upon the $n$ th-order operator (4.40) so that we obtain a symmetry of this theory. Since it has been shown that all coefficients are expressed in terms of the top component, we expect that this condition may be written as a closed form equation in $\zeta_{\alpha(n) \dot{\alpha}(n)}$.

In the massive case, the requirement that the symmetry operator $\mathfrak{O}^{(n)}$ preserves the equation of motion

$$
\Pi \mathfrak{O}^{(n)} \phi+m \overline{\left(\mathfrak{O}^{(n)} \phi\right)}=0,
$$

leads to new conditions which arise from setting the contributions proportional to the derivatives of $\bar{\phi}$ to zero. The most fundamental of these is

$$
\begin{aligned}
\mathcal{D}^{\beta} \overline{\mathcal{D}}^{\dot{\beta}} \zeta_{\beta \alpha(n-1) \dot{\beta} \dot{\alpha}(n-1)}= & 2(n+1) G^{\beta \dot{\beta}} \zeta_{\beta \alpha(n-1) \dot{\beta} \dot{\alpha}(n-1)} \\
& +\frac{2 \mathrm{i}(n+1)}{n}\left(\zeta_{\alpha(n-1) \dot{\alpha}(n-1)}-\bar{\zeta}_{\alpha(n-1) \dot{\alpha}(n-1)}\right) .
\end{aligned}
$$

It is more useful to work with an expression only in terms of the top component. Substituting (4.44) into (5.23) yields the Killing condition

$$
\mathcal{D}^{\beta} \overline{\mathcal{D}}^{\dot{\beta}} \zeta_{\beta \alpha(n-1) \dot{\beta} \dot{\alpha}(n-1)}=2 n(n+1) G^{\beta \dot{\beta}} \zeta_{\beta \alpha(n-1) \dot{\beta} \dot{\alpha}(n-1)},
$$

which implies

$$
\mathcal{D}^{\beta \dot{\beta}} \zeta_{\beta \alpha(n-1) \dot{\beta} \dot{\alpha}(n-1)}=0
$$

Fixing $n=1$, we obtain the well-known Killing condition for supervector fields (1.3).

In the case of AdS superspace $\operatorname{AdS}^{4 \mid 4}, G_{\alpha \dot{\alpha}}=0$ and the Killing condition (5.24) reduces to (1.4b) originally described in [49]. Given two Killing tensor superfields $\zeta_{\alpha(m) \dot{\alpha}(m)}^{1}$ and $\zeta_{\alpha(n) \dot{\alpha}(n)}^{2}$ in $\operatorname{AdS}^{4 \mid 4}$, the bracket (4.63) coincides with the one presented in [49].

\section{Bosonic backgrounds}

Similar to general relativity, of special interest are supergravity backgrounds which support unbroken symmetries. In the context of supersymmetric field theory we are primarily interested in those backgrounds which possess some amount of unbroken supersymmetry. This naturally leads us to restrict our attention to so-called bosonic backgrounds. By definition such a supergravity background has no covariant fermionic fields,

$$
\mathcal{D}_{\alpha} R\left|=0, \quad \mathcal{D}_{\alpha} G_{\beta \dot{\beta}}\right|=0, \quad W_{\alpha \beta \gamma}\left|=0, \quad X_{\alpha}\right|=0,
$$

where the bar projection is defined as in eq. (B.1). These conditions imply that the gravitino can be gauged away. In the remainder of this section we will assume that the gravitino is absent. We will also make use of the Weyl multiplet gauge described in appendix C.

Since there are no background fermionic fields, it follows from the equations (3.4) that every conformal Killing supervector field can uniquely be written as a sum of even and odd ones. A conformal Killing supervector field $\xi^{A}$ is called even if

$$
v^{a}(x):=\xi^{a}\left|\neq 0, \quad \xi^{\alpha}\right|=0 .
$$


A conformal Killing supervector field $\xi^{A}$ is called odd provided

$$
\xi^{a}\left|=0, \quad \epsilon^{\alpha}(x):=\xi^{\alpha}\right| \neq 0 .
$$

All information about the even and odd conformal Killing supervector fields is encoded in the vector $v^{a}$ and spinor $\epsilon^{\alpha}$ fields, respectively.

\subsection{Conformal isometries}

In this section we make extensive use of the component field formalism reviewed in appendix B and work within the Weyl multiplet gauge constructed in appendix C. Since the gravitino has been gauged away, which is possible due to (6.1), the component torsion tensor (C.4b) vanishes, which leaves us with a torsionless Lorentz connection. The component covariant derivative is

$$
\mathcal{D}_{a} \mid=\mathfrak{D}_{a}, \quad\left[\mathfrak{D}_{a}, \mathfrak{D}_{b}\right]=\frac{1}{2} R_{a b} c d M_{c d}+\mathrm{i} F_{a b} \mathbb{A} .
$$

where the Lorentz curvature and $\mathrm{U}(1)_{R}$ field strength take the form

$$
\begin{aligned}
R_{a b c d}= & \frac{1}{2}\left(\sigma_{a b}\right)^{\alpha \beta}\left(\sigma_{c d}\right)^{\gamma \delta} \mathcal{D}_{(\alpha} W_{\beta \gamma \delta)}\left|-\frac{1}{2}\left(\tilde{\sigma}_{a b}\right)^{\dot{\alpha} \dot{\beta}}\left(\tilde{\sigma}_{c d}\right)^{\dot{\gamma} \dot{\delta}} \overline{\mathcal{D}}_{(\dot{\alpha}} \bar{W}_{\dot{\beta} \dot{\gamma} \dot{\delta})}\right| \\
& +\frac{1}{4}\left(\left(\tilde{\sigma}_{a b}\right)^{\dot{\alpha} \dot{\beta}}\left(\sigma_{c d}\right)^{\alpha \beta}+\left(\sigma_{a b}\right)^{\alpha \beta}\left(\tilde{\sigma}_{c d}\right)^{\dot{\alpha} \dot{\beta}}\right) \mathcal{D}_{(\alpha} \overline{\mathcal{D}}_{(\dot{\alpha}} G_{\beta) \dot{\beta})} \mid \\
& -\frac{1}{24}\left(\eta_{c[a} \eta_{b] d}-\eta_{d[a} \eta_{b] c}\right) \mathcal{D}^{\alpha} X_{\alpha} \mid, \\
F_{a b}= & \frac{\mathrm{i}}{8}\left(\sigma_{a b}\right)^{\alpha \beta} \mathcal{D}_{\alpha} X_{\beta}\left|-\frac{\mathrm{i}}{8}\left(\tilde{\sigma}_{a b}\right)^{\dot{\alpha} \dot{\beta}} \overline{\mathcal{D}}_{\dot{\alpha}} \bar{X}_{\dot{\beta}}\right| .
\end{aligned}
$$

When working with a $\mathrm{U}(1)_{R}$ neutral field $\psi(x)$, it holds that $\mathfrak{D}_{a} \psi=\nabla_{a} \psi$, where

$$
\nabla_{a}:=\mathfrak{D}_{a}-\mathrm{i} \varphi_{a} \mathbb{A}
$$

is the torsion-free Lorentz-covariant derivative.

In section 3, we derived the necessary conditions on the transformation parameters $\boldsymbol{\Xi}$, eq. (3.13), associated with a conformal Killing supervector field $\xi^{A}$. Here, we wish to extract from these conditions all the restrictions on even and odd conformal Killing supervector fields. These are readily derivable by bar projecting the results for $\mathcal{D}_{a} \boldsymbol{\Xi}$.

Let $\xi^{A}$ be an even conformal Killing supervector field. Making use of the definitions (B.12) and bar projecting eq. (3.8b) leads to

$$
\nabla_{a} v_{b}=k_{a b}[v]+\eta_{a b} \sigma[v],
$$

which implies

$$
k_{a b}[v]=\nabla_{[a} v_{b]}, \quad \sigma[v]=\frac{1}{4} \nabla_{a} v^{a} .
$$

We see that $v^{a}$ is a conformal Killing vector field,

$$
\nabla_{(a} v_{b)}=\frac{1}{4} \eta_{a b} \nabla_{c} v^{c}
$$


Further, one may show that every conformal Killing vector field on $\mathcal{M}^{4}$ may be lifted to a unique even conformal Killing supervector field on $\mathcal{M}^{4 \mid 4}$. It should be remarked that the $\mathrm{U}(1)_{R}$ parameter $\varrho[v]$ is given by

$$
\nabla_{a} \varrho[v]=-F_{a b} v^{b} .
$$

Let $\xi^{A}$ be an odd conformal Killing supervector field, eq. (6.3). Then the bar projection of (3.8a) yields the conformal Killing spinor equation

$$
\mathfrak{D}_{\alpha \dot{\alpha}} \epsilon_{\beta}=-\mathrm{i} \varepsilon_{\alpha \beta} \bar{\eta}_{\dot{\alpha}}[\epsilon],
$$

where we have defined $\eta_{\alpha}:=\mathcal{D}_{\alpha} \Sigma \mid$. The equivalent form of this equation is

$$
\mathfrak{D}_{(\alpha \dot{\alpha}} \epsilon_{\beta)}=0 \text {. }
$$

\subsection{Isometries of old minimal supergravity backgrounds}

Let $\xi=\xi^{B} E_{B}$ be a conformal Killing supervector field on $\left(\mathcal{M}^{4 \mid 4}, \mathcal{D}\right)$, eq. (5.1a). We recall that the transformation $\delta_{\mathcal{K}[\xi]}+\delta_{\Sigma[\xi]}$ is said to be an isometry if the conformal compensator is left invariant, eq. (5.1b). In general, this requirement leads to severe restrictions on the symmetry parameters. Here, we will investigate the case of old minimal supergravity.

By making use of the Weyl, local $\mathrm{U}(1)_{R}$ and S-supersymmetry transformations we are able to adopt the gauge

$$
S_{0}\left|=1, \quad \mathcal{D}_{\alpha} S_{0}\right|=0 .
$$

This leaves us with a single component field which cannot be gauged away

$$
M:=-\frac{1}{4} \mathcal{D}^{2} S_{0} \mid
$$

As we have fixed the local $\mathrm{U}(1)_{R}$ invariance in this gauge, it is more convenient to work with the Lorentz-covariant derivative (6.6).

We find that in the case of an even symmetry, equation $(5.1 \mathrm{~b})$ is equivalent to the conditions

$$
\sigma[v]=0, \quad \varrho[v]=v^{a} \varphi_{a}, \quad v^{a} \nabla_{a} M=0 .
$$

As a result, (6.7) reduces to

$$
\nabla_{a} v_{b}=k_{a b}[v] \quad \Longrightarrow \quad \nabla_{(a} v_{b)}=0
$$

and therefore $v^{a}$ is a Killing vector field.

If we instead consider odd symmetries, we obtain

$$
\eta_{\alpha}[\epsilon]=-M \epsilon_{\alpha}-\frac{2}{3} \bar{\epsilon}^{\dot{\alpha}} \varphi_{\alpha \dot{\alpha}} .
$$

Thus, we are able to obtain from (6.11) the Killing spinor equation

$$
\nabla_{\alpha \dot{\alpha}} \epsilon_{\beta}=\mathrm{i} \varphi_{(\alpha \dot{\alpha}} \epsilon_{\beta)}+\mathrm{i} \varepsilon_{\alpha \beta}\left(\bar{M} \bar{\epsilon}_{\dot{\alpha}}+\frac{1}{6} \varphi_{\gamma \dot{\alpha}} \epsilon^{\gamma}\right)
$$

which was originally given in [18]. 


\subsection{Isometries of new minimal supergravity backgrounds}

In the case of new minimal supergravity, the conformal compensator $L$ is a linear multiplet, eq. (5.12). Associated with $L$ is the real vector descendant

$$
L_{\alpha \dot{\alpha}}:=-\frac{1}{2}\left[\mathcal{D}_{\alpha}, \overline{\mathcal{D}}_{\dot{\alpha}}\right] L+G_{\alpha \dot{\alpha}} L
$$

with the important property

$$
\mathcal{D}^{\alpha \dot{\alpha}} L_{\alpha \dot{\alpha}}=\frac{\mathrm{i}}{2}\left(\bar{X}_{\dot{\alpha}}+3 \overline{\mathcal{D}}_{\dot{\alpha}} \bar{R}\right) \overline{\mathcal{D}}^{\dot{\alpha}} L-\frac{\mathrm{i}}{2}\left(X^{\alpha}+3 \mathcal{D}^{\alpha} R\right) \mathcal{D}_{\alpha} L .
$$

Working in the Weyl multiplet gauge, the freedom to perform the Weyl and Ssupersymmetry transformations allows us to impose the additional gauge conditions

$$
L\left|=1, \quad \mathcal{D}_{\alpha} L\right|=0 .
$$

Owing to the reality of $L$, we stay with unbroken $\mathrm{U}(1)_{R}$ transformations. The only remaining component field of $L$ is

$$
H_{\alpha \dot{\alpha}}:=L_{\alpha \dot{\alpha}} \mid
$$

Making use of (6.20), we arrive at the constraint

$$
\nabla^{a} H_{a}=0 .
$$

Considering the case of an even symmetry, equation (5.1b) leads to

$$
\sigma[v]=0, \quad v^{b} \nabla_{b} H_{a}=0 .
$$

As a result, the Killing vector equation is given by

$$
\nabla_{a} v_{b}=k_{a b}[v] \quad \Longrightarrow \quad \nabla_{(a} v_{b)}=0 .
$$

In the case of odd symmetries, we deduce the charged Killing spinor equation

$$
\eta_{\alpha}[\epsilon]=-\frac{1}{2} H_{\alpha \dot{\beta}} \bar{\epsilon}^{\dot{\beta}} \quad \Longrightarrow \quad \mathfrak{D}_{\alpha \dot{\alpha}} \epsilon_{\beta}=\frac{\mathrm{i}}{2} \varepsilon_{\alpha \beta} H_{\gamma \dot{\alpha}} \epsilon^{\gamma},
$$

which is equivalent to the one originally derived in [18].

\subsection{Components of the (conformal) Killing tensor superfields}

Given a primary tensor field $t_{\alpha(p) \dot{\alpha}(q)}$ on a curved spacetime, we say that it is conformal Killing if it satisfies

$$
\mathfrak{D}_{\alpha \dot{\alpha}} t_{\alpha(p) \dot{\alpha}(q)}=0 \text {. }
$$

Further, it is said to be Killing if

$$
\mathfrak{D}^{\beta \dot{\beta}} t_{\beta \alpha(p-1) \dot{\beta} \alpha(q-1)} .
$$


Consider a conformal Killing tensor superfield $\ell_{\alpha(m) \dot{\alpha}(n)}$ on $\mathcal{M}^{4 \mid 4}$ with $m \geq 1$ and $n \geq 1$. It obeys the constraints (4.3a) and (4.3b). At the component level it contains four independent fields:

$$
\begin{aligned}
K_{\alpha(m) \dot{\alpha}(n)} & :=\ell_{\alpha(m) \dot{\alpha}(n)} \mid, \\
M_{\alpha(m-1) \dot{\alpha}(n)} & :=\mathcal{D}^{\beta} \ell_{\beta \alpha(m-1) \dot{\alpha}(n)} \mid, \\
N_{\alpha(m) \dot{\alpha}(n-1)} & :=\overline{\mathcal{D}}^{\dot{\beta}} \ell_{\alpha(m) \dot{\beta} \dot{\alpha}(n-1)} \mid, \\
L_{\alpha(m-1) \dot{\alpha}(n-1)} & :=\left[\mathcal{D}^{\beta}, \overline{\mathcal{D}}^{\dot{\beta}}\right] \ell_{\beta \alpha(m-1) \dot{\beta} \dot{\alpha}(n-1)} \mid .
\end{aligned}
$$

By a straightforward calculation, we find that each component field defines a conformal Killing tensor field on the background in the sense of (6.27). In the special case where $\ell_{\alpha(m) \dot{\alpha}(n)}$ is Killing, it is easily shown that these component fields also satisfy the Killing condition (6.28).

\subsection{Components of conformal supercurrents}

A primary tensor field $t^{\alpha(p) \dot{\alpha}(q)}$ on a curved spacetime will be called a conserved current if it satisfies the divergenceless condition

$$
\mathfrak{D}_{\beta \dot{\beta}} t^{\beta \alpha(p-1) \dot{\beta} \dot{\alpha}(q-1)}=0 .
$$

Given a conformal supercurrent $J^{\alpha(m) \dot{\alpha}(n)}$, eq. (4.15), it contains four independent component fields, which can be chosen as follows (an implicit symmetrisation over all $\alpha$ indices and, independently, all $\dot{\alpha}$-indices is assumed)

$$
\begin{aligned}
j^{\alpha(m) \dot{\alpha}(n)} & :=J^{\alpha(m) \dot{\alpha}(n)} \mid, \\
Q^{\alpha(m+1) \dot{\alpha}(n)} & :=\mathcal{D}^{\alpha} J^{\alpha(m) \dot{\alpha}(n)} \mid, \\
S^{\alpha(m) \dot{\alpha}(n+1)} & :=\overline{\mathcal{D}}^{\dot{\alpha}} J^{\alpha(m) \dot{\alpha}(n)} \mid, \\
T^{\alpha(m+1) \dot{\alpha}(n+1)} & :=\left[\mathcal{D}^{\alpha}, \overline{\mathcal{D}}^{\dot{\alpha}}\right] J^{\alpha(m) \dot{\alpha}(n)} \mid .
\end{aligned}
$$

It is easily verified that $j^{\alpha(m) \dot{\alpha}(n)}, Q^{\alpha(m+1) \dot{\alpha}(n)}$ and $S^{\alpha(m) \dot{\alpha}(n+1)}$ define conserved currents satisfying eq. (6.30) for an arbitrary background. This is true for $T^{\alpha(m+1) \dot{\alpha}(n+1)}$ only in the special case where $m=n=1$. Let us elaborate on the current (6.31d) in some more detail.

In the case of AdS and Minkowski superspace backgrounds, $T^{\alpha(m+1) \dot{\alpha}(n+1)}$ may always be improved,

$$
\mathbb{T}^{\alpha(m+1) \dot{\alpha}(n+1)}:=T^{\alpha(m+1) \dot{\alpha}(n+1)}-\frac{2 \mathrm{i}(m-n)}{m+n+2} \mathfrak{D}^{\alpha \dot{\alpha}} j^{\alpha(m) \dot{\alpha}(n)},
$$

to give a conserved current, $\mathfrak{D}_{\beta \dot{\beta}} \mathbb{T}^{\beta \alpha(m) \dot{\beta} \dot{\alpha}(n)}=0$, for arbitrary positive integers $m$ and $n$. Since the supercurrent $J^{\alpha(m) \dot{\alpha}(n)}$ is a primary superfield, it should be always possible to improve (6.31d) to a conserved current in a conformally flat background, $C_{a b c d}=0$. However, if the background Weyl tensor is non-vanishing, $C_{a b c d} \neq 0$, it is not possible to improve $T^{\alpha(m+1) \dot{\alpha}(n+1)}$ to a conserved current provided $m>1$ and/or $n>1$. This conclusion is analogous to a recent result of Beccaria and Tseytlin [94] who demonstrated 
that for a conformal scalar field in curved space there is no way to construct a conserved traceless symmetric spin-3 current $\mathcal{J}^{a b c}$ if the background Weyl tensor is non-vanishing.

Next, we consider conformal supercurrents of the form $J^{\alpha(m)}$ (4.17). At the component level, it contains two possible candidates for conserved currents:

$$
\begin{aligned}
j^{\alpha(m) \dot{\alpha}} & :=\overline{\mathcal{D}}^{\dot{\alpha}} J^{\alpha(m)} \mid, \\
T^{\alpha(m+1) \dot{\alpha}} & :=\left[\mathcal{D}^{\alpha}, \overline{\mathcal{D}}^{\dot{\alpha}}\right] J^{\alpha(m)} \mid .
\end{aligned}
$$

A routine calculation reveals that $j^{\alpha(m) \dot{\alpha}}$ does indeed constitute a conserved current. In the context of AdS and Minkowski superspaces, it is always possible to extend $T^{\alpha(m) \dot{\alpha}}$ to a conserved current by setting $m=0$ in (6.32), however this fails in the general case.

The final case of interest is that of a scalar conformal supercurrent $J$ (4.18a). It contains a single current at the component level,

$$
T^{\alpha \dot{\alpha}}:=\left[\mathcal{D}^{\alpha}, \overline{\mathcal{D}}^{\dot{\alpha}}\right] J \mid, \quad \mathfrak{D}_{\alpha \dot{\alpha}} T^{\alpha \dot{\alpha}}=0,
$$

which is conserved for any curved background.

\subsection{Maximally supersymmetric backgrounds}

There exist only five maximally supersymmetric backgrounds in off-shell $4 \mathrm{D} \mathcal{N}=1$ supergravity, as was first demonstrated by Festuccia and Seiberg [18] in the component setting. There is a remarkably simple superspace derivation of this classification $[95,96]$ which we review here. Unlike the previous analysis in this section, which has relied on the Weyl multiplet gauge, this derivation makes use of the gauge condition (5.7).

We start by recalling an important theorem concerning the maximally supersymmetric backgrounds $[22,25]$. For any supergravity theory in $D$ dimensions formulated in superspace, all maximally supersymmetric spacetimes correspond to those supergravity backgrounds which are characterised by the following properties: (i) all Grassmann-odd components of the superspace torsion and curvature tensors vanish; and (ii) all Grassmann-even components of the torsion and curvature tensors are annihilated by the spinor derivatives.

In the case of $4 \mathrm{D} \mathcal{N}=1$ supergravity, the above theorem means the following:

$$
\begin{aligned}
X_{\alpha} & =0, \\
W_{\alpha \beta \gamma} & =0, \\
\mathcal{D}_{\alpha} R=0 \Longrightarrow \mathcal{D}_{A} R & =0, \\
\mathcal{D}_{\alpha} G_{\beta \dot{\beta}}=0 \Longrightarrow \mathcal{D}_{A} G_{\beta \dot{\beta}} & =0 .
\end{aligned}
$$

Equation (6.35a) tells us that all maximally supersymmetric backgrounds are realised in terms of the GWZ geometry [4,5]. Equation (6.35b) tells us that all maximally supersymmetric backgrounds are conformally flat. Equations $(6.35 \mathrm{c})$ and $(6.35 \mathrm{~d})$ restrict $R$ and $G_{\beta \dot{\beta}}$ to be covariantly constant. Equation (6.35d) has an integrability condition that follows from

$$
0=\left\{\overline{\mathcal{D}}_{\dot{\alpha}}, \overline{\mathcal{D}}_{\dot{\beta}}\right\} G_{\gamma \dot{\gamma}}=4 R \varepsilon_{\dot{\gamma}(\dot{\alpha}} G_{\gamma \dot{\beta})},
$$


and therefore we obtain the constraint

$$
R G_{\alpha \dot{\alpha}}=0
$$

There is an alternative way to arrive at this constraint. Relation (6.35d) tells us that $G_{\beta \dot{\beta}}$ satisfies the superconformal Killing equation (3.5b), and therefore the condition (3.6) holds. Since $G_{\beta \dot{\beta}}$ is covariantly constant, (3.6) reduces to (6.37).

The simplest solution to (6.37) is $R=0$ and $G_{\alpha \dot{\alpha}}=0$, which corresponds to Minkowski superspace. Another solution is described by $G_{\alpha \dot{\alpha}}=0$ and $R=\mu \neq 0$, which corresponds to the AdS superspace (4.50). The three remaining superspaces are characterised by formally identical anti-commutation relations

$$
\begin{array}{rlrl}
\left\{\mathcal{D}_{\alpha}, \mathcal{D}_{\beta}\right\} & =0, & \left\{\overline{\mathcal{D}}_{\dot{\alpha}}, \overline{\mathcal{D}}_{\dot{\beta}}\right\}=0, \quad\left\{\mathcal{D}_{\alpha}, \overline{\mathcal{D}}_{\dot{\beta}}\right\}=-2 \mathrm{i} \mathcal{D}_{\alpha \dot{\beta}}, \\
{\left[\mathcal{D}_{\alpha}, \mathcal{D}_{\beta \dot{\beta}}\right]} & =\mathrm{i} \varepsilon_{\alpha \beta} G_{\dot{\beta}}^{\gamma} \mathcal{D}_{\gamma}, & {\left[\overline{\mathcal{D}}_{\dot{\alpha}}, \mathcal{D}_{\beta \dot{\beta}}\right]=-\mathrm{i} \varepsilon_{\dot{\alpha} \dot{\beta}} G_{\beta}{ }^{\dot{\gamma}} \overline{\mathcal{D}}_{\dot{\gamma}},} \\
{\left[\mathcal{D}_{\alpha \dot{\alpha}}, \mathcal{D}_{\beta \dot{\beta}}\right]} & =-\mathrm{i} \varepsilon_{\dot{\alpha} \dot{\beta}} G_{\beta}{ }^{\dot{\gamma}} \mathcal{D}_{\alpha \dot{\gamma}}+\mathrm{i} \varepsilon_{\alpha \beta} G_{\dot{\beta}}^{\gamma} \mathcal{D}_{\gamma \dot{\alpha}},
\end{array}
$$

where $G_{b}$ is covariantly constant, $\mathcal{D}_{A} G_{b}=0$. The difference between these superspaces is encoded in the Lorentzian type of $G_{a}$. Since $G^{2}=G^{a} G_{a}$ is constant, the geometry (6.38) describes three different superspaces, $\mathbb{M}_{T}^{4 \mid 4}, \mathbb{M}_{S}^{4 \mid 4}$ and $\mathbb{M}_{N}^{4 \mid 4}$, which correspond to the choices $G^{2}<0, G^{2}>0$ and $G^{2}=0$, respectively. The Lorentzian manifolds, which are the bosonic bodies of the superspaces $\mathbb{M}_{T}^{4 \mid 4}, \mathbb{M}_{S}^{4 \mid 4}$ and $\mathbb{M}_{N}^{4 \mid 4}$, are $\mathbb{R} \times S^{3}, \mathrm{AdS}_{3} \times \mathbb{R}$ and a pp-wave spacetime, respectively. The latter spacetime is isometric to the so-called Nappi-Witten group [97], as shown in [98].

Each superspace (6.38) is maximally supersymmetric solution of $R^{2}$ supergravity [96].

\section{Conclusion}

To conclude this paper we summarise the main results obtained and list several interesting open problems. The main outcomes of this work include the following.

- We described the general structure of (conformal) isometries of supergravity backgrounds within the $\mathrm{U}(1)$ superspace setting. Using the formalism developed, it is trivial to read off the known (conformal) Killing spinor equations for unbroken supersymmetry transformations. What is more important is that our formalism makes it possible to reconstruct, starting from a given (conformal) Killing spinor field, a unique (conformal) Killing supervector field which generates the corresponding supersymmetry transformation on $\mathcal{M}^{4 \mid 4}$.

- It was shown that the infinitesimal (conformal) isometry transformations form a closed algebra for any supergravity background.

- We introduced the (conformal) Killing tensor superfields $\ell_{\alpha(m)(\dot{\alpha}(n)}$, where $m$ and $n$ non-negative integers, $m+n>0$, and demonstrated their significance in the following cases: (i) $m=n$, with the choice $n=1$ corresponding to the (conformal) isometries; 
and (ii) $m-1=n=0$. In particular, we showed that extended (conformal) supersymmetry transformations are formulated in terms of the (conformal) Killing spinor superfields $\ell_{\alpha}$. It was proved that the conformal Killing tensor superfields with $m=n$ generate all (non-trivial) symmetries of the massless Wess-Zumino operator and form a superalgebra with respect to the bracket (4.63). In the case of conformally flat superspaces this leads to a geometric realisation of the $\mathcal{N}=1$ conformal higher-spin superalgebra $[99,100] .{ }^{11}$

- We introduced the conformal supercurrents $J^{\alpha(m) \dot{\alpha}(n)}$ of arbitrary valence $(m, n)$ in a supergravity background and analysed their component structure.

Interesting open problems include the following.

- We believe that all coefficients of the symmetry operator $\mathfrak{O}^{(n)}$, eq. $(4.40)$, can be expressed in terms of the top component $\zeta^{\alpha(n) \dot{\alpha}(n)}$. We have been able to prove this for the lowest cases $n=1,2$. It would be interesting to extend the proof to greater values of $n$. In the case of Minkowski superspace, this was proved in [58].

- We expect that the component field defined by (6.31d) can be improved to a conserved current $\mathbb{T}^{\alpha(m+1) \dot{\alpha}(n+1)}(m, n \geq 0)$, on any conformally flat bosonic background. A proof of this result would be important. Perhaps the best approach to address this problem is to make use of conformal superspace [40]. ${ }^{12}$

- It would be interesting to extend the analysis of section 4 to off-shell supergravity backgrounds in diverse dimensions. In particular, it is an interesting problem to describe the higher symmetries of a massless hypermultiplet in $4 \mathrm{D} \mathcal{N}=2$ conformal supergravity backgrounds.

- It would be interesting to make use of the techniques developed in our paper to identify supersymmetric models whose symmetries are generated by the superconformal Killing-Yano tensor introduced by Howe and Lindström [59].

- As an extension of Eastwood's influential work [47], there have appeared several publications on higher symmetries of the conformal powers of the Laplacian including $[102-105] .{ }^{13}$ It would be interesting to carry out a similar analysis for the $\mathcal{N}=1$ and $\mathcal{N}=2$ superconformal extensions of $\square^{2}$ proposed in $[109,110]$.

- General non-conformal deformations of the conformal supercurrents $J^{\alpha(n) \dot{\alpha}(n)}$ and $J^{\alpha(n+1) \dot{\alpha}(n)}$, eq. (4.15), were described in $[72,111,112]$ for the cases of Minkowski and AdS backgrounds. Various aspects of such non-conformal higher-spin supercurrents

\footnotetext{
${ }^{11}$ All conformal higher-spin superalgebras in four dimensions were classified in [101]. These results were extended to higher dimensions in [48].

${ }^{12}$ While proof-reading the manuscript, we were able to prove this claim by deriving a closed form expression for the divergence of this improved component field as a function of the background Weyl tensor and the lower spin current $j^{\alpha(m) \dot{\alpha}(n)}$. A complete proof will be described elsewhere.

${ }^{13}$ The symmetry algebras for higher-derivative equations such as $\square^{n}$ were actually introduced in the bulk language in [106]. See also [107, 108] for further developments.
} 
in Minkowski superspace were studied in [113-115]. It would be interesting to study consistent non-conformal deformations of other conformal supercurrents introduced in section 4.2 .

\section{Acknowledgments}

Conversations and email correspondence with Misha Vasiliev are gratefully acknowledged. The work of SK is supported in part by the Australian Research Council, project No. DP200101944. The work of ER is supported by the Hackett Postgraduate Scholarship UWA, under the Australian Government Research Training Program.

\section{A Chiral action}

There is an alternative way to define the chiral action (3.24) that follows from the superform approach to the construction of supersymmetric invariants [116-120]. It is based on the use of the following super four-form

$$
\begin{aligned}
\Xi_{4}\left[\mathcal{L}_{\mathrm{c}}\right]= & 2 \mathrm{i} \bar{E}_{\dot{\delta}} \wedge \bar{E}_{\dot{\gamma}} \wedge E^{b} \wedge E^{a}\left(\tilde{\sigma}_{a b}\right)^{\dot{\gamma} \dot{\delta}} \mathcal{L}_{\mathrm{c}}+\frac{\mathrm{i}}{6} \varepsilon_{a b c d} \bar{E}_{\dot{\delta}} \wedge E^{c} \wedge E^{b} \wedge E^{a}\left(\tilde{\sigma}^{d}\right)^{\dot{\delta} \delta} \mathcal{D}_{\delta} \mathcal{L}_{\mathrm{c}} \\
& -\frac{1}{96} \varepsilon_{a b c d} E^{d} \wedge E^{c} \wedge E^{b} \wedge E^{a}\left(\mathcal{D}^{2}-12 \bar{R}\right) \mathcal{L}_{\mathrm{c}}
\end{aligned}
$$

which was constructed by Binétruy et al. [121] and independently by Gates et al. [120]. ${ }^{14}$ Here we have made use of the superspace vielbein

$$
E^{A}=\left(E^{a}, E^{\alpha}, \bar{E}_{\dot{\alpha}}\right)=\mathrm{d} z^{M} E_{M}^{A} .
$$

These super one-forms constitute the dual basis to $E_{A}=\left(E_{a}, E_{\alpha}, \bar{E}^{\dot{\alpha}}\right)=E_{A}=E_{A}{ }^{M} \partial_{M}$. The super four-form (A.1) is closed,

$$
\mathrm{d} \Xi_{4}\left[\mathcal{L}_{\mathrm{c}}\right]=0
$$

The chiral action $(3.24)$ can be recast as an integral of $\Xi_{4}\left[\mathcal{L}_{\mathrm{c}}\right]$ over a spacetime $\mathcal{M}^{4}$,

$$
S_{\mathrm{c}}=\int_{\mathcal{M}^{4}} \Xi_{4}\left[\mathcal{L}_{\mathrm{c}}\right]
$$

where $\mathcal{M}^{4}$ is the bosonic body of the curved superspace $\mathcal{M}^{4 \mid 4}$ obtained by switching off the Grassmann variables. The representation (A.4) provides the simplest way to reduce the action from superfields to components.

Making use of the super-Weyl transformation laws

$$
\delta_{\Sigma} E^{a}=-\Sigma E^{a}, \quad \delta_{\Sigma} E^{\alpha}=-\frac{1}{2} \Sigma E^{\alpha}-\frac{\dot{\mathrm{i}}}{2} \overline{\mathcal{D}}_{\dot{\beta}} E^{b}\left(\tilde{\sigma}_{b}\right)^{\dot{\beta} \alpha},
$$

it may be shown that the super four-form (A.1) is super-Weyl invariant. This result extends the analysis given in [123] where the GWZ geometry was used. In conformal superspace [40] the superform (A.1) was described in [124].

\footnotetext{
${ }^{14} \mathrm{~A}$ simple derivation of (A.1), based on the use of an on-shell vector multiplet, was given in [122].
} 


\section{B Component reduction}

To study supergravity-matter theories at the component level, it is necessary to make use of the technique of bar projection. Given a superfield $\Xi(z)$ defined on $\mathcal{M}^{4 \mid 4}$, we define

$$
\Xi|(x):=\Xi(x, \theta, \bar{\theta})|_{\theta^{\mu}=\bar{\theta}_{\dot{\mu}}=0} .
$$

Thus, $\Xi \mid$ is a field defined on the background spacetime $\mathcal{M}^{4}$. In the same way, we may define the bar projection of a covariant derivative by bar projecting the connection superfields

$$
\mathcal{D}_{A}\left|:=E_{A}{ }^{M}\right| \partial_{M}+\frac{1}{2} \Omega_{A}{ }^{b c}\left|M_{b c}+\mathrm{i} \Phi_{A}\right| \mathbb{A} .
$$

In particular, the bar projected vector covariant derivative takes the form

$$
\mathcal{D}_{a}\left|=\mathfrak{D}_{a}+\frac{1}{2} \psi_{a}{ }^{\beta} \mathcal{D}_{\beta}\right|+\frac{1}{2} \bar{\psi}_{a, \dot{\beta}} \overline{\mathcal{D}}^{\dot{\beta}} \mid,
$$

where we have introduced both the gravitino $\psi_{a}{ }^{\beta}$ and the charged spacetime covariant derivative

$$
\mathfrak{D}_{a}=e_{a}+\frac{1}{2} \omega_{a}^{b c} M_{b c}+\mathrm{i} \varphi_{a} \mathbb{A} .
$$

\section{B.1 Wess-Zumino gauge}

By making use of the $\mathcal{K}$ gauge freedom (2.5), we are able to fix a Wess-Zumino gauge on the spinor covariant derivatives

$$
\mathcal{D}_{\alpha}\left|=\delta_{\alpha}{ }^{\mu} \partial_{\mu}, \quad \overline{\mathcal{D}}^{\dot{\alpha}}\right|=\delta^{\dot{\alpha}}{ }_{\dot{\mu}} \bar{\partial}^{\dot{\mu}} .
$$

This gauge leads to the useful identities

$$
E_{a}{ }^{m}\left|=e_{a}{ }^{m}, \quad E_{a}{ }^{\mu}\right|=\frac{1}{2} \psi_{a}{ }^{\beta} \delta_{\beta}{ }^{\mu}, \quad \Omega_{a}{ }^{b c}\left|=\omega_{a}{ }^{b c}, \quad \Phi_{a}\right|=\varphi_{a} .
$$

In what follows, we will adopt gauge (B.5).

Naturally, we are interested in determining the residual gauge transformations which preserve the conditions (B.5). These must satisfy the identity

$$
\left(\delta_{\mathcal{K}}+\delta_{\Sigma}\right) \mathcal{D}_{\alpha} \mid=0
$$

The $\mathcal{K}$ gauge transformations act on the components of the connection by the rules:

$$
\begin{aligned}
\delta_{\mathcal{K}} E_{A}{ }^{M}= & \xi^{B} \mathcal{T}_{B A}{ }^{C} E_{C}{ }^{M}-\left(\mathcal{D}_{A} \xi^{B}\right) E_{B}{ }^{M}+K_{A}{ }^{B} E_{B}{ }^{M}+\mathrm{i} \rho w_{A}{ }^{B} E_{B}{ }^{M}, \\
\delta_{\mathcal{K}} \Omega_{A}{ }^{c d}= & \xi^{B} \mathcal{T}_{B A}{ }^{E} \Omega_{E}{ }^{c d}+\xi^{B} \mathcal{R}_{B A}{ }^{c d}-\left(\mathcal{D}_{A} \xi^{B}\right) \Omega_{B}{ }^{c d}+K_{A}{ }^{B} \Omega_{B}{ }^{c d}-\mathcal{D}_{A} K^{c d} \\
& +\mathrm{i} \rho w_{A}{ }^{B} \Omega_{B}{ }^{c d} \\
\delta_{\mathcal{K}} \Phi_{A}= & \xi^{B} \mathcal{T}_{B A}{ }^{C} \Phi_{C}+\xi^{B} \mathcal{F}_{B A}-\left(\mathcal{D}_{A} \xi^{B}\right) \Phi_{B}+K_{A}{ }^{B} \Phi_{B}+\mathrm{i} \rho w_{A}{ }^{B} \Phi_{B}-\mathcal{D}_{A} \rho .
\end{aligned}
$$

Where we have introduced

$$
K_{A}{ }^{B}=\left(\begin{array}{ccc}
K_{a}{ }^{b} & 0 & 0 \\
0 & K_{\alpha}{ }^{\beta} & 0 \\
0 & 0 & -\bar{K}^{\dot{\alpha}}{ }_{\dot{\beta}}
\end{array}\right), \quad w_{A}{ }^{B}=\left(\begin{array}{ccc}
0 & 0 & 0 \\
0 & -\delta_{\alpha}{ }^{\beta} & 0 \\
0 & 0 & \delta^{\dot{\alpha}} \dot{\beta}
\end{array}\right) .
$$


By making use of (2.12), we extract the super-Weyl transformation laws for the connections:

$$
\begin{aligned}
\delta_{\Sigma} E_{\alpha}{ }^{M}= & \frac{\Sigma}{2} E_{\alpha}{ }^{M}, \\
\delta_{\Sigma} \Omega_{\alpha}{ }^{c d}= & \frac{\Sigma}{2} \Omega_{\alpha}{ }^{c d}+2\left(\sigma^{c d}\right)_{\alpha \beta} \mathcal{D}^{\beta} \Sigma, \\
\delta_{\Sigma} \Phi_{\alpha}= & \frac{\Sigma}{2} \Phi_{\alpha}-\frac{3 \mathrm{i}}{2} \mathcal{D}_{\alpha} \Sigma, \\
\delta_{\Sigma} E_{a}{ }^{M}= & \Sigma E_{a}{ }^{M}-\frac{\mathrm{i}}{2}\left(\tilde{\sigma}_{a}\right)^{\dot{\alpha} \alpha} \mathcal{D}_{\alpha} \Sigma \bar{E}_{\dot{\alpha}}{ }^{M}-\frac{\mathrm{i}}{2}\left(\tilde{\sigma}_{a}\right)^{\dot{\alpha} \alpha} \overline{\mathcal{D}}_{\dot{\alpha}} \Sigma E_{\alpha}{ }^{M}, \\
\delta_{\Sigma} \Omega_{a}{ }^{c d}= & \Sigma \Omega_{a}{ }^{c d}-\frac{\mathrm{i}}{2}\left(\tilde{\sigma}_{a}\right)^{\dot{\alpha} \alpha} \mathcal{D}_{\alpha} \Sigma \bar{\Omega}_{\dot{\alpha}}{ }^{c d}-\frac{\mathrm{i}}{2}\left(\tilde{\sigma}_{a}\right)^{\dot{\alpha} \alpha} \overline{\mathcal{D}}_{\dot{\alpha}} \Sigma \Omega_{\alpha}{ }^{c d}+\delta_{a}{ }^{[c} \mathcal{D}^{d]} \Sigma \\
& +\frac{1}{4} \varepsilon_{a}{ }^{b c d}\left(\tilde{\sigma}_{b}\right)^{\dot{\alpha} \alpha}\left[\mathcal{D}_{\alpha}, \overline{\mathcal{D}}_{\dot{\alpha}}\right] \Sigma, \\
\delta_{\Sigma} \Phi_{a}= & \Sigma \Phi_{a}-\frac{\mathrm{i}}{2}\left(\tilde{\sigma}_{a}\right)^{\dot{\alpha} \alpha} \mathcal{D}_{\alpha} \Sigma \bar{\Phi}_{\dot{\alpha}}-\frac{\mathrm{i}}{2}\left(\tilde{\sigma}_{a}\right)^{\dot{\alpha} \alpha} \overline{\mathcal{D}}_{\dot{\alpha}} \Sigma \Phi_{\alpha}+\frac{3}{8}\left(\tilde{\sigma}_{a}\right)^{\dot{\alpha} \alpha}\left[\mathcal{D}_{\alpha}, \overline{\mathcal{D}}_{\dot{\alpha}}\right] \Sigma .
\end{aligned}
$$

Thus, (B.7) takes the form

$$
\begin{aligned}
\mathcal{D}_{\alpha} \xi^{\beta} \mid & =\xi^{C} \mathcal{T}_{C \alpha}{ }^{\beta}\left|+K_{\alpha}{ }^{\beta}\right|-\mathrm{i} \delta_{\alpha}{ }^{\beta} \rho\left|+\frac{1}{2} \delta_{\alpha}{ }^{\beta} \Sigma\right|, \\
\mathcal{D}_{\alpha} \bar{\xi}_{\dot{\beta}} \mid & =\xi^{C} \mathcal{T}_{C \alpha, \dot{\beta}} \mid, \\
\mathcal{D}_{\alpha} \xi^{b} \mid & =\xi^{C} \mathcal{T}_{C \alpha}{ }^{b} \mid \\
\mathcal{D}_{\alpha} K^{c d} \mid & =\xi^{B} \mathcal{R}_{B \alpha}{ }^{c d}\left|-2\left(\sigma^{c d}\right)_{\alpha}{ }^{\beta} \mathcal{D}_{\beta} \Sigma\right|, \\
\mathcal{D}_{\alpha} \rho \mid & =\xi^{B} \mathcal{F}_{B \alpha}\left|-\frac{3 \mathrm{i}}{2} \mathcal{D}_{\alpha} \Sigma\right| .
\end{aligned}
$$

Note that these are equivalent to the bar projection of the conformal Killing conditions (3.4). These place severe restrictions on the transformations which preserve this gauge. In particular only the following gauge parameters remain unconstrained

$$
v^{a}:=\xi^{a}\left|, \quad \epsilon^{\alpha}:=\xi^{\alpha}\right|, \quad k_{a b}:=K_{a b}|, \quad \varrho:=\rho|,
$$

which correspond to general coordinate, local $Q$-supersymmetry, Lorentz and $\mathrm{U}(1)_{R}$ transformations respectively.

\section{B.2 Component field strengths}

The (charged) spacetime covariant derivative introduced in (B.4) obeys the following commutation relations

$$
\left[\mathfrak{D}_{a}, \mathfrak{D}_{b}\right]=T_{a b}{ }^{c} \mathfrak{D}_{c}+\frac{1}{2} R_{a b}{ }^{c d} M_{c d}+\mathrm{i} F_{a b} \mathbb{A},
$$

where $T_{a b}{ }^{c}$ is the torsion, $R_{a b}{ }^{c d}$ is the Lorentz curvature and $F_{a b}$ is the $\mathrm{U}(1)_{R}$ field strength. By making use of (B.3) and the bar projection of (2.8e) it is possible to read off the field strengths.

The simplest field strength to compute is the torsion

$$
T_{a b c}=-\frac{\mathrm{i}}{2}\left(\psi_{a} \sigma_{c} \bar{\psi}_{b}-\psi_{b} \sigma_{c} \bar{\psi}_{a}\right)-\varepsilon_{a b c d} G^{d} \mid .
$$


This result allows us to decompose the Lorentz connection in terms of a torsionless (spin) connection and the torsion

$$
\omega_{a b c}=\omega_{a b c}(e)+\frac{1}{2}\left(T_{a b c}-T_{b c a}+T_{c a b}\right) .
$$

It is also convenient to introduce the gravitino field strength

$$
\Psi_{a b}^{\gamma}:=\mathfrak{D}_{a} \psi_{b}{ }^{\gamma}-\mathfrak{D}_{b} \psi_{a}{ }^{\gamma}-T_{a b}{ }^{c} \psi_{c}{ }^{\gamma},
$$

which can be computed to be

$$
\begin{aligned}
\Psi_{a b}{ }^{\gamma}= & -\mathrm{i} \psi_{[a}{ }^{\alpha}\left(\sigma_{b]}\right)_{\alpha \dot{\alpha}} G^{\dot{\alpha} \gamma}\left|-\mathrm{i} \bar{\psi}_{[a, \dot{\alpha}}\left(\tilde{\sigma}_{b]}\right)^{\dot{\alpha} \gamma} R\right| \\
& -\frac{\mathrm{i}}{2}\left(\tilde{\sigma}_{a b}\right)^{\dot{\alpha} \dot{\beta}} \psi_{\alpha \dot{\alpha}}{ }^{\gamma} G^{\alpha}{ }_{\dot{\beta}}\left|-\frac{\mathrm{i}}{2}\left(\sigma_{a b}\right)^{\alpha \beta} \psi_{\alpha \dot{\lambda},}{ }^{\gamma} G_{\beta}{ }^{\dot{\lambda}}\right|+\left(\sigma_{a b}\right)^{\alpha \beta} W_{\alpha \beta}{ }^{\gamma} \mid \\
& +\frac{1}{6}\left(\sigma_{a b}\right)^{\gamma \alpha} X_{\alpha}\left|+\frac{1}{2}\left(\sigma_{a b}\right)^{\gamma \alpha} \mathcal{D}_{\alpha} R\right|+\frac{1}{2}\left(\tilde{\sigma}_{a b}\right)^{\dot{\alpha} \dot{\beta}} \overline{\mathcal{D}}_{(\dot{\alpha}} G^{\gamma}{ }_{\dot{\beta})} \mid
\end{aligned}
$$

Next, the $\mathrm{U}(1)_{R}$ field strength is given by

$$
F_{a b}=\frac{1}{8}\left(\sigma_{a b}\right)^{\alpha \beta}\left(\mathrm{i} \mathcal{D}_{(\alpha} X_{\beta)}\left|+\bar{\psi}_{(\alpha \dot{\alpha},},^{\dot{\alpha}} X_{\beta)}\right|-\psi_{(\alpha \dot{\alpha}, \beta)} \bar{X}^{\dot{\alpha}} \mid\right)+\text { c.c. . }
$$

Finally, we compute the Lorentz curvature

$$
\begin{aligned}
R_{a b c d}= & \frac{1}{2}\left(\mathrm{i} \eta_{d e} \eta_{c[a}-\mathrm{i} \eta_{c e} \eta_{d[a}+\varepsilon_{c d e[a}\right) \psi_{b]},{ }^{\alpha}\left(\sigma^{e}\right)_{\alpha \dot{\alpha}} \overline{\mathcal{D}}^{\dot{\alpha}} \bar{R}\left|-\frac{\mathrm{i}}{2}\left(\tilde{\sigma}_{[a}\right)^{\dot{\alpha} \alpha} \psi_{b], \alpha}\left(\sigma_{c d}\right)^{\gamma \delta} \mathcal{D}_{\gamma} G_{\delta \dot{\alpha}}\right| \\
& +\mathrm{i}\left(\tilde{\sigma}_{[a}\right)^{\dot{\alpha} \alpha} \bar{\psi}_{b], \dot{\alpha}}\left(\sigma_{c d}\right)^{\beta \gamma} W_{\alpha \beta \gamma}\left|+\frac{1}{12}\left(\mathrm{i} \eta_{d e} \eta_{c[a}-\mathrm{i} \eta_{c e} \eta_{d[a}+\varepsilon_{c d e[a}\right) \bar{\psi}_{b], \dot{\alpha}}\left(\tilde{\sigma}^{e}\right)^{\dot{\alpha} \dot{\alpha}} X_{\alpha}\right| \\
& +\frac{1}{16}\left(\eta_{a c} \eta_{b d}-\eta_{a d} \eta_{b c}+\mathrm{i} \varepsilon_{a b c d}\right)\left(\overline{\mathcal{D}}^{2} \bar{R}|-8 R| \bar{R} \mid\right)+\psi_{a} \sigma_{c d} \psi_{b} \bar{R} \mid \\
& -\frac{1}{4}\left(\tilde{\sigma}_{a b}\right)^{\dot{\alpha} \dot{\beta}}\left(\sigma_{c d}\right)^{\alpha \beta} \overline{\mathcal{D}}_{\dot{\alpha}} \mathcal{D}_{\alpha} G_{\beta \dot{\beta}}\left|+\frac{1}{2}\left(\sigma_{a b}\right)^{\alpha \beta}\left(\sigma_{c d}\right)^{\gamma \delta} \mathcal{D}_{\alpha} W_{\beta \gamma \delta}\right| \\
& +\frac{1}{48}\left(\eta_{d e} \eta_{c[a}-\eta_{c e} \eta_{d[a}+\mathrm{i} \varepsilon_{c d e[a}\right)\left(\sigma_{b]} \tilde{\sigma}^{e}\right)^{\alpha \beta} \mathcal{D}_{\alpha} X_{\beta} \mid+ \text { c.c. }
\end{aligned}
$$

When working at the component level, it is often necessary to understand the relationship between the irreducible components of these field strengths and the component structure of the torsion superfields and their derivatives.

We begin with an analysis of the gravitino field strength $\Psi_{a b}{ }^{\gamma}$, which yields

$$
\begin{aligned}
& \mathcal{D}_{\alpha} R\left|+\frac{1}{3} X_{\alpha}\right|=-\frac{4}{3} \Psi_{\alpha}{ }^{\beta}{ }_{, \beta}+6 \mathrm{i} \psi^{\beta \dot{\alpha}}{ }_{, \alpha} G_{\beta) \dot{\alpha}}\left|-9 \mathrm{i} \bar{\psi}_{\alpha}{ }_{,{ }_{,}}{ }_{\alpha} R\right|, \\
& W_{\alpha \beta \gamma}\left|=\Psi_{(\alpha \beta, \gamma)}-\mathrm{i} \psi_{(\alpha, \beta}{ }^{\dot{\alpha}} G_{\gamma) \dot{\alpha}}\right|, \\
& \overline{\mathcal{D}}_{(\dot{\alpha}} G_{\beta \dot{\beta})}\left|=-2 \Psi_{\dot{\alpha} \dot{\beta}, \beta}-\mathrm{i} \psi_{\beta(\dot{\alpha},}{ }^{\alpha} G_{\alpha \dot{\beta})}\right|+\mathrm{i} \bar{\psi}_{\beta(\dot{\alpha}, \dot{\beta})} R \mid \text {. }
\end{aligned}
$$

Moving on to the $\mathrm{U}(1)_{R}$ field strength $F_{a b}$, we have a single irreducible component

$$
\mathcal{D}_{(\alpha} X_{\beta)}\left|=-8 \mathrm{i} F_{\alpha \beta}+8 \mathrm{i} \bar{\psi}_{(\alpha \dot{\alpha}},{ }^{\dot{\alpha}} X_{\beta)}\right|-8 \mathrm{i} \psi_{(\alpha \dot{\alpha}, \beta)} \bar{X}^{\dot{\alpha}} \mid \text {. }
$$


The remaining relations arise from the Lorentz curvature $R_{a b c d}$

$$
\begin{aligned}
\mathcal{D}^{2} R \mid= & \frac{2}{3}\left(R(e, \psi)-\frac{\mathrm{i}}{2} \varepsilon_{a b c d} R^{a b c d}\right)+2 \mathrm{i} \bar{\psi}^{\alpha \dot{\alpha}}{ }_{, \dot{\alpha}} \mathcal{D}_{\alpha} R\left|+\frac{2 \mathrm{i}}{3} \bar{\psi}^{\alpha \dot{\alpha}, \dot{\beta}} \overline{\mathcal{D}}_{(\dot{\alpha}} G_{\alpha \dot{\beta})}\right| \\
& -3 \mathrm{i} \psi^{\alpha \dot{\alpha}}{ }_{, \alpha} \bar{X}_{\dot{\alpha}}\left|+\frac{2}{3} \bar{\psi}^{\alpha \dot{\alpha}}{ }_{,(\dot{\alpha}} \bar{\psi}_{\alpha}{ }^{\dot{\beta}}{ }_{, \dot{\beta})} R\right|+8 R|\bar{R}|-\frac{1}{3} \mathcal{D} X \mid \\
\overline{\mathcal{D}}_{(\dot{\alpha}} \mathcal{D}_{(\alpha} G_{\beta) \dot{\beta})} \mid= & 2 E_{\alpha \beta, \dot{\alpha} \dot{\beta}}+\mathrm{i} \psi^{\gamma}{ }_{(\dot{\alpha}, \gamma} \mathcal{D}_{(\alpha} G_{\beta) \dot{\beta})}\left|-2 \mathrm{i} \bar{\psi}^{\gamma}{ }_{(\dot{\alpha}, \dot{\beta})} W_{\alpha \beta \gamma}\right|+\frac{\mathrm{i}}{3} \bar{\psi}_{(\alpha(\dot{\alpha}, \dot{\beta})} X_{\beta)} \mid \\
& -\psi^{\gamma}{ }_{(\dot{\alpha},(\alpha} \psi_{\gamma \dot{\beta}), \beta)} \bar{R}\left|+2 \mathrm{i} \psi_{(\alpha(\dot{\alpha}, \beta)} \overline{\mathcal{D}}_{\dot{\beta})} \bar{R}\right|, \\
\mathcal{D}_{(\alpha} W_{\beta \gamma \delta)} \mid= & C_{\alpha \beta \gamma \delta}+\mathrm{i} \psi_{(\alpha \dot{\alpha}, \beta} \bar{\Psi}_{\gamma \delta),}{ }^{\dot{\alpha}}+\mathrm{i} \bar{\psi}_{(\alpha \dot{\alpha},}{ }^{\dot{\alpha}} \Psi_{\beta \gamma \delta)}-\bar{\psi}_{\left(\alpha \dot{\alpha},{ }^{\dot{\alpha}} \psi_{\beta \dot{\beta}, \gamma} G_{\delta)}{ }^{\dot{\beta}} \mid\right.} \\
& -2 \bar{\psi}_{(\alpha \dot{\alpha}, \dot{\beta}} \psi_{\beta}{ }_{, \gamma}{ }_{, \gamma} G_{\delta)}{ }^{\dot{\beta}} \mid
\end{aligned}
$$

Where we have defined

$$
R(e, \psi)=\eta^{a c} \eta^{b d} R_{a b c d}, \quad E_{\alpha \beta, \dot{\alpha} \dot{\beta}}=\frac{1}{2} R_{(\dot{\alpha}, \gamma \dot{\beta}), \alpha \beta}^{\gamma}, \quad C_{\alpha \beta \gamma \delta}=\frac{1}{2} R_{(\alpha}^{\alpha_{, \beta \dot{\alpha}, \gamma \delta)}} .
$$

It is well known that $C_{\alpha \beta \gamma \delta}$ is the spinor form of the anti-self-dual part of the usual Weyl tensor and as a result $W_{\alpha \beta \gamma}$ is often referred to as the 'super Weyl tensor'. Similarly, $E_{\alpha \beta, \dot{\alpha} \dot{\beta}}$ coincides with the traceless component of the Ricci tensor and so we say that $G_{\alpha \dot{\alpha}}$ is its supersymmetric extension.

\section{The Weyl multiplet gauge}

It is often advantageous to adopt a gauge which partially fixes the super-Weyl freedom (2.12) in exchange for gauging several (component) fields to zero. We recall that in $\mathrm{U}(1)$ superspace this freedom is parametrised by a real scalar superfield $\Sigma$ (2.12), thus it contains six independent component fields in its multiplet.

The component fields $\sigma:=\Sigma \mid$ and $\eta_{\alpha}:=\mathcal{D}_{\alpha} \Sigma \mid$ parametrise Weyl and S-supersymmetry transformations, respectively. Recalling equations (2.13), we observe that by making use of our freedom in the $\mathcal{D}^{2} \Sigma \mid$ and $\left[\mathcal{D}_{\alpha}, \overline{\mathcal{D}}_{\dot{\alpha}}\right] \Sigma \mid$ component fields, it is possible to adopt a gauge where $R|=\bar{R}|=0$ and $G_{\alpha \dot{\alpha}} \mid=0$. Further, by a routine calculation one can derive

$$
\begin{aligned}
\delta_{\Sigma}\left(\mathcal{D}_{\alpha} R\right)= & \frac{3}{2} \Sigma \mathcal{D}_{\alpha} R+4 \mathcal{D}_{\alpha} \Sigma R+\frac{1}{2} \mathcal{D}_{\alpha} \overline{\mathcal{D}}^{2} \Sigma \\
\delta_{\Sigma}\left(\mathcal{D}^{2} R\right)= & 2 \Sigma \mathcal{D}^{2} R+4 \mathcal{D}^{2} \Sigma R+4 \mathcal{D}^{\alpha} \Sigma \mathcal{D}_{\alpha} R+\frac{1}{2} \mathcal{D}^{2} \overline{\mathcal{D}}^{2} \Sigma \\
\delta_{\Sigma}\left(\mathcal{D}^{2} R+\overline{\mathcal{D}}^{2} \bar{R}\right)= & 2 \Sigma\left(\mathcal{D}^{2} R+\overline{\mathcal{D}}^{2} \bar{R}\right)+4 \overline{\mathcal{D}}^{2} \Sigma \bar{R}+4 \mathcal{D}^{2} \Sigma R \\
& +4 \mathcal{D}^{\alpha} \Sigma \mathcal{D}_{\alpha} R+4 \overline{\mathcal{D}}_{\dot{\alpha}} \Sigma \overline{\mathcal{D}}^{\dot{\alpha}} \bar{R}+\frac{1}{2}\left\{\mathcal{D}^{2}, \overline{\mathcal{D}}^{2}\right\} \Sigma
\end{aligned}
$$

By making use of the $R \mid=0$ gauge condition, it is possible to use the freedom in $\mathcal{D}_{\alpha} \overline{\mathcal{D}}^{2} \Sigma \mid$ to fix $\mathcal{D}_{\alpha} R \mid=0$. Finally, we can further extend the gauge by using $\left\{\mathcal{D}^{2}, \overline{\mathcal{D}}^{2}\right\} \Sigma \mid$ to set $\mathcal{D}^{2} R\left|+\overline{\mathcal{D}}^{2} \bar{R}\right|=0$. This completes our gauge fixing procedure. 
We must also determine the residual combined gauge (2.6) and super-Weyl transformations which preserve this gauge. A routine computation leads to the conditions

$$
\begin{aligned}
\mathcal{D}^{2} \Sigma \mid= & 0 \\
{\left[\mathcal{D}_{\alpha}, \overline{\mathcal{D}}_{\dot{\alpha}}\right] \Sigma \mid=} & -\left(\xi^{B} \mathcal{D}_{B} G_{\alpha \dot{\alpha}}\right) \mid \\
\mathcal{D}_{\alpha} \overline{\mathcal{D}}^{2} \Sigma \mid= & -2\left(\xi^{B} \mathcal{D}_{B} \mathcal{D}_{\alpha} R\right) \mid \\
\left\{\mathcal{D}^{2}, \overline{\mathcal{D}}^{2}\right\} \Sigma \mid= & \left(\xi^{\alpha \dot{\alpha}} \mathcal{D}_{\alpha \dot{\alpha}}\left(\mathcal{D}^{2} R+\overline{\mathcal{D}}^{2} \bar{R}\right)\right)\left|+8 \mathrm{i}\left(\bar{\xi}^{\dot{\alpha}} \mathcal{D}_{\alpha \dot{\alpha}} \mathcal{D}^{\alpha} R\right)\right| \\
& +8 \mathrm{i}\left(\xi^{\alpha} \mathcal{D}_{\alpha \dot{\alpha}} \overline{\mathcal{D}}^{\dot{\alpha}} \bar{R}\right) \mid .
\end{aligned}
$$

In summary, adopting the Weyl multiplet gauge has allowed us to fix

$$
R\left|=0, \quad G_{\alpha \dot{\alpha}}\right|=0, \quad \mathcal{D}_{\alpha} R\left|=0, \quad \mathcal{D}^{2} R\right|+\overline{\mathcal{D}}^{2} \bar{R} \mid=0,
$$

while retaining unbroken Weyl $\sigma$ and S-supersymmetry transformations $\eta_{\alpha}$.

Now, we return to our discussion of the field strengths (B.14), (B.17), (B.18) and (B.19). By imposing (C.3), we find that these take the simplified form

$$
\begin{aligned}
T_{a b c}= & -\frac{\mathrm{i}}{2}\left(\psi_{a} \sigma_{c} \bar{\psi}_{b}-\psi_{b} \sigma_{c} \bar{\psi}_{a}\right), \\
\Psi_{a b,}{ }^{\gamma}= & \left(\sigma_{a b}\right)^{\alpha \beta} W_{\alpha \beta}{ }^{\gamma}\left|+\frac{1}{6}\left(\sigma_{a b}\right)^{\gamma \alpha} X_{\alpha}\right|+\frac{1}{2}\left(\tilde{\sigma}_{a b}\right)^{\dot{\alpha} \dot{\beta}} \overline{\mathcal{D}}_{\dot{\alpha}} G_{\dot{\beta}}^{\gamma} \mid, \\
R_{a b c d}= & -\frac{\mathrm{i}}{2}\left(\tilde{\sigma}_{[a}\right)^{\dot{\alpha} \alpha} \psi_{b], \alpha}\left(\sigma_{c d}\right)^{\gamma \delta} \mathcal{D}_{\gamma} G_{\delta \dot{\alpha}}\left|+\mathrm{i}\left(\tilde{\sigma}_{[a}\right)^{\dot{\alpha} \alpha} \bar{\psi}_{b], \dot{\alpha}}\left(\sigma_{c d}\right)^{\beta \gamma} W_{\alpha \beta \gamma}\right| \\
& +\frac{1}{12}\left(\mathrm{i} \eta_{d e} \eta_{c[a}-\mathrm{i} \eta_{c e} \eta_{d[a}+\varepsilon_{c d e[a}\right) \bar{\psi}_{b], \dot{\alpha}}\left(\tilde{\sigma}^{e}\right)^{\dot{\alpha} \alpha} X_{\alpha}\left|+\frac{\mathrm{i}}{16} \varepsilon_{a b c d} \overline{\mathcal{D}}^{2} \bar{R}\right| \\
& -\frac{1}{4}\left(\tilde{\sigma}_{a b}\right)^{\dot{\alpha} \dot{\beta}}\left(\sigma_{c d}\right)^{\alpha \beta} \overline{\mathcal{D}}_{\dot{\alpha}} \mathcal{D}_{\alpha} G_{\beta \dot{\beta}}\left|+\frac{1}{2}\left(\sigma_{a b}\right)^{\alpha \beta}\left(\sigma_{c d}\right)^{\gamma \delta} \mathcal{D}_{\alpha} W_{\beta \gamma \delta}\right| \\
& +\frac{1}{48}\left(\eta_{d e} \eta_{c[a}-\eta_{c e} \eta_{d[a}+\mathrm{i} \varepsilon_{c d e[a}\right)\left(\sigma_{b]} \tilde{\sigma}^{e}\right)^{\alpha \beta} \mathcal{D}_{\alpha} X_{\beta} \mid+ \text { c.c. }, \\
F_{a b}= & \frac{1}{8}\left(\sigma_{a b}\right)^{\alpha \beta}\left(\mathrm{i} \mathcal{D}_{(\alpha} X_{\beta)}\left|+\bar{\psi}_{\alpha \dot{\alpha}},{ }^{\dot{\alpha}} X_{\beta}\right|-\psi_{\alpha \dot{\gamma}, \beta} \bar{X}^{\dot{\gamma}} \mid\right)+\text { c.c. . }
\end{aligned}
$$

Another advantageous property of this choice of gauge is that the relations (B.20), (B.21) and (B.22) are greatly simplified. We read off

$$
\begin{aligned}
& X_{\alpha}\left|=-4 \Psi_{\alpha}{ }^{\beta}, \beta, \quad W_{\alpha \beta \gamma}\right|=\Psi_{(\alpha \beta, \gamma)}, \quad \overline{\mathcal{D}}_{(\dot{\alpha}} G_{\beta \dot{\beta})} \mid=-2 \Psi_{\dot{\alpha} \dot{\beta}, \beta}, \\
& \mathcal{D}_{(\alpha} X_{\beta)} \mid=-8 \mathrm{i} F_{\alpha \beta}+32 \mathrm{i} \bar{\psi}_{(\alpha}{ }^{\dot{\alpha}}{ }_{, \dot{\alpha}} \Psi_{\beta) \gamma}{ }^{\gamma}-32 \mathrm{i} \psi_{(\alpha}{ }^{\dot{\alpha}}{ }_{, \beta)} \bar{\Psi}_{\dot{\alpha}}{ }^{\dot{\beta}}{ }_{, \dot{\beta}}, \\
& \mathcal{D} X \mid=2 R(e, \psi)+\left(18 \mathrm{i} \psi_{, \alpha}^{\alpha \dot{\alpha}} \bar{\Psi}_{\dot{\alpha}}^{\dot{\beta}}{ }_{, \dot{\beta}}-2 \mathrm{i} \psi^{\alpha \dot{\alpha}, \beta} \bar{\Psi}_{\alpha \beta, \dot{\alpha}}+\text { c.c. }\right) \text {, } \\
& \mathcal{D}^{2} R \mid=-\frac{\mathrm{i}}{3} \varepsilon^{a b c d} R_{a b c d}+\mathrm{i}\left(6 \psi^{\alpha \dot{\alpha}}{ }_{, \alpha} \bar{\Psi}_{\dot{\alpha}}{ }_{, \dot{\beta}}{ }_{\dot{\beta}}+\frac{2}{3} \psi^{\alpha \dot{\alpha}, \beta} \bar{\Psi}_{\alpha \beta, \dot{\alpha}}+\text { c.c. }\right) \text {, } \\
& \mathcal{D}_{(\dot{\alpha}} \mathcal{D}_{(\alpha} G_{\beta) \dot{\beta})} \mid=2 E_{\alpha \beta, \dot{\alpha} \dot{\beta}}-2 \mathrm{i} \psi_{(\dot{\alpha}, \gamma}^{\gamma} \bar{\Psi}_{\alpha \beta, \dot{\beta})}-2 \mathrm{i} \bar{\psi}_{(\dot{\alpha}, \dot{\beta})}^{\gamma} \Psi_{(\alpha \beta, \gamma)}-\frac{4}{3} \mathrm{i} \bar{\psi}_{(\alpha(\dot{\alpha}, \beta)} \Psi_{\beta)}{ }^{\gamma} \gamma, \\
& \mathcal{D}_{(\alpha} W_{\beta \gamma \delta)} \mid=C_{\alpha \beta \gamma \delta}+\mathrm{i} \bar{\psi}_{(\alpha \dot{\alpha},}{ }^{\dot{\alpha}} \Psi_{\beta \gamma, \delta)}+\mathrm{i} \psi_{(\alpha \dot{\alpha}, \beta} \bar{\Psi}_{\gamma \delta)},{ }^{\dot{\alpha}} .
\end{aligned}
$$

When combined with the algebra (2.8) and Bianchi identites (2.11), these relations allow us to express all component fields of the torsion superfields in terms of the (component) field strengths. The main implication of this is that the only remaining independent 
component fields are the spacetime vielbein $e_{m}{ }^{a}$, the gravitino $\psi_{m}{ }^{\alpha}$ (and its conjugate) and the $\mathrm{U}(1)_{R}$ gauge field $\varphi_{m}$, which are known to comprise the Weyl multiplet.

The approach described in this appendix is analogous to the one used for $3 \mathrm{D} \mathcal{N}=2$ supergravity [23].

Open Access. This article is distributed under the terms of the Creative Commons Attribution License (CC-BY 4.0), which permits any use, distribution and reproduction in any medium, provided the original author(s) and source are credited.

\section{References}

[1] B. Zumino, Nonlinear Realization of Supersymmetry in de Sitter Space, Nucl. Phys. B 127 (1977) 189 [INSPIRE].

[2] E.A. Ivanov and A.S. Sorin, Superfield formulation of $\operatorname{OSP}(1,4)$ supersymmetry, J. Phys. A 13 (1980) 1159 [INSPIRE].

[3] I.L. Buchbinder and S.M. Kuzenko, Ideas and Methods of Supersymmetry and Supergravity or a Walk Through Superspace, IOP, Bristol U.K. (1995) [revised edition (1998)].

[4] R. Grimm, J. Wess and B. Zumino, Consistency Checks on the Superspace Formulation of Supergravity, Phys. Lett. B 73 (1978) 415 [InSPIRE].

[5] R. Grimm, J. Wess and B. Zumino, A Complete Solution of the Bianchi Identities in Superspace, Nucl. Phys. B 152 (1979) 255 [InSPIRE].

[6] J. Wess and B. Zumino, Superfield Lagrangian for Supergravity, Phys. Lett. B 74 (1978) 51 [INSPIRE].

[7] J. Wess and J. Bagger, Supersymmetry and Supergravity, Princeton University Press, Princeton U.S.A. (1992).

[8] W. Siegel, Supergravity superfields without a supermetric, Harvard preprint HUTP-77/A068 (1977).

[9] W. Siegel, A polynomial action for a massive, self-interacting chiral superfield coupled to supergravity, Harvard preprint HUTP-77/A077 (1977).

[10] W. Siegel, The superfield supergravity action, Harvard preprint HUTP-77/A080 (1977).

[11] W. Siegel, A derivation of the supercurrent superfield, Harvard preprint HUTP-77/A089 (1977).

[12] K.S. Stelle and P.C. West, Minimal Auxiliary Fields for Supergravity, Phys. Lett. 74B (1978) 330 [INSPIRE].

[13] S. Ferrara and P. van Nieuwenhuizen, The Auxiliary Fields of Supergravity, Phys. Lett. B 74 (1978) 333 [INSPIRE].

[14] M.F. Sohnius, The conformal group in superspace, in Quantum Theory and the Structures of Time and Space. Vol. 2, L. Castell, M. Drieschner and C.F. von`Weizsäcker eds., Carl Hanser Verlag, München Germany (1977), pg. 241.

[15] W. Lang, Construction of the Minimal Superspace Translation Tensor and the Derivation of the Supercurrent, Nucl. Phys. B 179 (1981) 106 [INSPIRE]. 
[16] L. Bonora, P. Pasti and M. Tonin, Cohomologies and Anomalies in Supersymmetric Theories, Nucl. Phys. B 252 (1985) 458 [InSPIRE].

[17] K.-i. Shizuya, Supercurrents and Superconformal Symmetry, Phys. Rev. D 35 (1987) 1848 [INSPIRE].

[18] G. Festuccia and N. Seiberg, Rigid Supersymmetric Theories in Curved Superspace, JHEP 06 (2011) 114 [arXiv: 1105.0689] [INSPIRE].

[19] B. Jia and E. Sharpe, Rigidly Supersymmetric Gauge Theories on Curved Superspace, JHEP 04 (2012) 139 [arXiv:1109.5421] [INSPIRE].

[20] H. Samtleben and D. Tsimpis, Rigid supersymmetric theories in 4d Riemannian space, JHEP 05 (2012) 132 [arXiv: 1203.3420] [INSPIRE].

[21] S.M. Kuzenko, Symmetries of curved superspace, JHEP 03 (2013) 024 [arXiv:1212.6179] [INSPIRE].

[22] S.M. Kuzenko, Supersymmetric spacetimes from curved superspace, PoS (CORFU2014) 140 [arXiv: 1504.08114] [INSPIRE].

[23] S.M. Kuzenko, U. Lindström, M. Roček, I. Sachs and G. Tartaglino-Mazzucchelli, Three-dimensional $\mathcal{N}=2$ supergravity theories: From superspace to components, Phys. Rev. D 89 (2014) 085028 [arXiv: 1312.4267] [INSPIRE].

[24] D. Butter, G. Inverso and I. Lodato, Rigid $4 D \mathcal{N}=2$ supersymmetric backgrounds and actions, JHEP 09 (2015) 088 [arXiv:1505.03500] [INSPIRE].

[25] S.M. Kuzenko, J. Novak and G. Tartaglino-Mazzucchelli, Symmetries of curved superspace in five dimensions, JHEP 10 (2014) 175 [arXiv:1406.0727] [INSPIRE].

[26] S.M. Kuzenko, U. Lindström and G. Tartaglino-Mazzucchelli, unpublished.

[27] S.M. Kuzenko and G. Tartaglino-Mazzucchelli, Five-dimensional $N=1$ AdS superspace: Geometry, off-shell multiplets and dynamics, Nucl. Phys. B 785 (2007) 34 [arXiv:0704.1185] [INSPIRE].

[28] S.M. Kuzenko and G. Tartaglino-Mazzucchelli, Field theory in $4 D N=2$ conformally flat superspace, JHEP 10 (2008) 001 [arXiv:0807.3368] [INSPIRE].

[29] D. Butter and S.M. Kuzenko, $N=2$ supersymmetric $\sigma$-models in AdS, Phys. Lett. B 703 (2011) 620 [arXiv: 1105.3111] [INSPIRE].

[30] D. Butter and S.M. Kuzenko, The structure of $N=2$ supersymmetric nonlinear $\sigma$-models in $A d S_{4}, J H E P 11$ (2011) 080 [arXiv:1108.5290] [INSPIRE].

[31] D. Butter, S.M. Kuzenko, U. Lindström and G. Tartaglino-Mazzucchelli, Extended supersymmetric $\sigma$-models in AdS $S_{4}$ from projective superspace, JHEP 05 (2012) 138 [arXiv: 1203.5001] [INSPIRE].

[32] S.M. Kuzenko and G. Tartaglino-Mazzucchelli, Three-dimensional $N=2$ (AdS) supergravity and associated supercurrents, JHEP 12 (2011) 052 [arXiv:1109.0496] [INSPIRE].

[33] S.M. Kuzenko, U. Lindström and G. Tartaglino-Mazzucchelli, Three-dimensional $(p, q) A d S$ superspaces and matter couplings, JHEP 08 (2012) 024 [arXiv:1205.4622] [INSPIRE].

[34] D. Butter, S.M. Kuzenko and G. Tartaglino-Mazzucchelli, Nonlinear $\sigma$-models with AdS supersymmetry in three dimensions, JHEP 02 (2013) 121 [arXiv:1210.5906] [INSPIRE]. 
[35] P.S. Howe, A superspace approach to extended conformal supergravity, Phys. Lett. B 100 (1981) 389 [INSPIRE].

[36] P.S. Howe, Supergravity in Superspace, Nucl. Phys. B 199 (1982) 309 [INSPIRE].

[37] S.J. Gates, M.T. Grisaru, M. Roček and W. Siegel, Superspace Or One Thousand and One Lessons in Supersymmetry, Front. Phys. 58 (1983) 1 [hep-th/0108200] [INSPIRE].

[38] M.F. Sohnius and P.C. West, An Alternative Minimal Off-Shell Version of $N=1$ Supergravity, Phys. Lett. B 105 (1981) 353 [INSPIRE].

[39] M. Sohnius and P.C. West, The Tensor Calculus and Matter Coupling of the Alternative Minimal Auxiliary Field Formulation of $N=1$ Supergravity, Nucl. Phys. B 198 (1982) 493 [INSPIRE].

[40] D. Butter, $N=1$ Conformal Superspace in Four Dimensions, Annals Phys. 325 (2010) 1026 [arXiv: 0906.4399] [INSPIRE].

[41] M. Walker and R. Penrose, On quadratic first integrals of the geodesic equations for type [22] spacetimes, Commun. Math. Phys. 18 (1970) 265 [INSPIRE].

[42] A. Mikhailov, Notes on higher spin symmetries, hep-th/0201019 [INSPIRE].

[43] A.G. Nikitin, Generalized Killing tensors of arbitrary rank and order, Ukr. Math. J. 43 (1991) 734 .

[44] A.G. Nikitin and O.I. Prylypko, Generalized Killing tensors and symmetry of Klein-Gordon-Fock equations, Preprint Akad. Nauk UkrSSR, Inst. Math., 90.26, Kiev Ukraine (1990), pg. 2 [math-ph/0506002].

[45] A.V. Shapovalov and I.V. Shirokov, Symmetry algebras of linear differential equations, Theor. Math. Phys. 92 (1992) 697 [Teor. Mat. Fiz. 92 (1992) 3].

[46] O.V. Shaynkman and M.A. Vasiliev, Higher spin conformal symmetry for matter fields in (2+1)-dimensions, Theor. Math. Phys. 128 (2001) 1155 [Teor. Mat. Fiz. 128 (2001) 378] [hep-th/0103208] [INSPIRE].

[47] M.G. Eastwood, Higher symmetries of the Laplacian, Annals Math. 161 (2005) 1645 [hep-th/0206233] [INSPIRE].

[48] M.A. Vasiliev, Higher spin superalgebras in any dimension and their representations, JHEP 12 (2004) 046 [hep-th/0404124] [INSPIRE].

[49] S.J. Gates, Jr., S.M. Kuzenko and A.G. Sibiryakov, Towards a unified theory of massless superfields of all superspins, Phys. Lett. B 394 (1997) 343 [hep-th/9611193] [INSPIRE].

[50] S.M. Kuzenko and A.G. Sibiryakov, Free massless higher superspin superfields on the anti-de Sitter superspace, Phys. Atom. Nucl. 57 (1994) 1257 [Yad. Fiz. 57 (1994) 1326] [arXiv:1112.4612] [INSPIRE].

[51] S.M. Kuzenko, A.G. Sibiryakov and V.V. Postnikov, Massless gauge superfields of higher half integer superspins, JETP Lett. 57 (1993) 534 [Pisma Zh. Eksp. Teor. Fiz. 57 (1993) 521] [INSPIRE].

[52] E.S. Fradkin and M.A. Vasiliev, Candidate to the Role of Higher Spin Symmetry, Annals Phys. 177 (1987) 63 [INSPIRE].

[53] E.S. Fradkin and M.A. Vasiliev, Superalgebra of Higher Spins and Auxiliary Fields, Int. J. Mod. Phys. A 3 (1988) 2983 [inSPIRE]. 
[54] M.A. Vasiliev, Extended Higher Spin Superalgebras and Their Realizations in Terms of Quantum Operators, Fortsch. Phys. 36 (1988) 33 [INSPIRE].

[55] S.E. Konshtein and M.A. Vasiliev, Massless Representations and Admissibility Condition for Higher Spin Superalgebras, Nucl. Phys. B 312 (1989) 402 [inSPIRE].

[56] S.E. Konstein and M.A. Vasiliev, Extended Higher Spin Superalgebras and Their Massless Representations, Nucl. Phys. B 331 (1990) 475 [INSPIRE].

[57] P.S. Howe and U. Lindström, Notes on Super Killing Tensors, JHEP 03 (2016) 078 [arXiv: 1511.04575] [INSPIRE].

[58] P.S. Howe and U. Lindström, Super-Laplacians and their symmetries, JHEP 05 (2017) 119 [arXiv: 1612.06787] [INSPIRE].

[59] P.S. Howe and U. Lindström, Some remarks on (super)-conformal Killing-Yano tensors, JHEP 11 (2018) 049 [arXiv: 1808.00583] [INSPIRE].

[60] D. Butter and S.M. Kuzenko, A dual formulation of supergravity-matter theories, Nucl. Phys. B 854 (2012) 1 [arXiv: 1106.3038] [INSPIRE].

[61] W. Siegel, Solution to Constraints in Wess-Zumino Supergravity Formalism, Nucl. Phys. B 142 (1978) 301 [INSPIRE].

[62] P.S. Howe and R.W. Tucker, Scale Invariance in Superspace, Phys. Lett. 80B (1978) 138 [INSPIRE].

[63] S.M. Kuzenko and G. Tartaglino-Mazzucchelli, Different representations for the action principle in $4 D N=2$ supergravity, JHEP 04 (2009) 007 [arXiv:0812.3464] [INSPIRE].

[64] G.W. Gibbons and P. Rychenkova, Cones, triSasakian structures and superconformal invariance, Phys. Lett. B 443 (1998) 138 [hep-th/9809158] [INSPIRE].

[65] R. Penrose and W. Rindler, Spinors And Space-time. Vol. 2: Spinor and Twistor Methods in Space-time Geometry, Cambridge University Press, Cambridge U.K. (1986).

[66] S.M. Kuzenko, R. Manvelyan and S. Theisen, Off-shell superconformal higher spin multiplets in four dimensions, JHEP 07 (2017) 034 [arXiv:1701.00682] [INSPIRE].

[67] S.M. Kuzenko and M. Ponds, Conformal geometry and (super)conformal higher-spin gauge theories, JHEP 05 (2019) 113 [arXiv:1902.08010] [INSPIRE].

[68] E.S. Fradkin and A.A. Tseytlin, Conformal supergravity, Phys. Rept. 119 (1985) 233 [INSPIRE].

[69] Y. Korovin, S.M. Kuzenko and S. Theisen, The conformal supercurrents in diverse dimensions and conserved superconformal currents, JHEP 05 (2016) 134 [arXiv: 1604.00488] [INSPIRE].

[70] S. Ferrara and B. Zumino, Transformation Properties of the Supercurrent, Nucl. Phys. B 87 (1975) 207 [INSPIRE].

[71] P.S. Howe, K.S. Stelle and P.K. Townsend, Supercurrents, Nucl. Phys. B 192 (1981) 332 [INSPIRE].

[72] E.I. Buchbinder, J. Hutomo and S.M. Kuzenko, Higher spin supercurrents in anti-de Sitter space, JHEP 09 (2018) 027 [arXiv: 1805.08055] [INSPIRE].

[73] S.M. Kuzenko and S. Theisen, Correlation functions of conserved currents in $N=2$ superconformal theory, Class. Quant. Grav. 17 (2000) 665 [hep-th/9907107] [INSPIRE]. 
[74] M.F. Sohnius, The Multiplet of Currents for $N=2$ Extended Supersymmetry, Phys. Lett. 81B (1979) 8 [INSPIRE].

[75] S. Ferrara, J. Wess and B. Zumino, Supergauge Multiplets and Superfields, Phys. Lett. B 51 (1974) 239 [INSPIRE].

[76] U. Lindström and M. Roček, New HyperKähler Metrics and New Supermultiplets, Commun. Math. Phys. 115 (1988) 21 [INSPIRE].

[77] U. Lindström and M. Roček, $N=2$ SuperYang-Mills Theory in Projective Superspace, Commun. Math. Phys. 128 (1990) 191 [InSPIRE].

[78] S.M. Kuzenko, On compactified harmonic/projective superspace, $5-D$ superconformal theories and all that, Nucl. Phys. B 745 (2006) 176 [hep-th/0601177] [InSPIRE].

[79] S.M. Kuzenko, On superconformal projective hypermultiplets, JHEP 12 (2007) 010 [arXiv:0710.1479] [INSPIRE].

[80] S.M. Kuzenko and G. Tartaglino-Mazzucchelli, Super-Weyl invariance in $5 D$ supergravity, JHEP 04 (2008) 032 [arXiv:0802.3953] [INSPIRE].

[81] S.M. Kuzenko, U. Lindström, M. Roček and G. Tartaglino-Mazzucchelli, On conformal supergravity and projective superspace, JHEP 08 (2009) 023 [arXiv: 0905.0063] [INSPIRE].

[82] S.M. Kuzenko, U. Lindström and G. Tartaglino-Mazzucchelli, Off-shell supergravity-matter couplings in three dimensions, JHEP 03 (2011) 120 [arXiv:1101.4013] [INSPIRE].

[83] B. de Wit, M. Roček and S. Vandoren, Hypermultiplets, hyperKähler cones and quaternion Kähler geometry, JHEP 02 (2001) 039 [hep-th/0101161] [INSPIRE].

[84] S.M. Kuzenko, $N=2$ supersymmetric $\sigma$-models and duality, JHEP 01 (2010) 115 [arXiv: 0910.5771] [INSPIRE].

[85] C.M. Hull, A. Karlhede, U. Lindström and M. Roček, Nonlinear $\sigma$ Models and Their Gauging in and Out of Superspace, Nucl. Phys. B 266 (1986) 1 [INSPIRE].

[86] M.A. Vasiliev, Nonlinear equations for symmetric massless higher spin fields in (A)dS(d), Phys. Lett. B 567 (2003) 139 [hep-th/0304049] [INSPIRE].

[87] W. Siegel and S.J. Gates, Jr., Superfield Supergravity, Nucl. Phys. B 147 (1979) 77 [INSPIRE].

[88] B. de Wit and M. Roček, Improved tensor multiplets, Phys. Lett. B 109 (1982) 439 [INSPIRE].

[89] S. Ferrara, L. Girardello, T. Kugo and A. Van Proeyen, Relation Between Different Auxiliary Field Formulations of $N=1$ Supergravity Coupled to Matter, Nucl. Phys. B 223 (1983) 191 [INSPIRE].

[90] P. Breitenlohner, A Geometric Interpretation of Local Supersymmetry, Phys. Lett. B 67 (1977) 49 [INSPIRE].

[91] P. Breitenlohner, Some Invariant Lagrangians for Local Supersymmetry, Nucl. Phys. B 124 (1977) 500 [INSPIRE].

[92] W. Siegel, Gauge Spinor Superfield as a Scalar Multiplet, Phys. Lett. B 85 (1979) 333 [INSPIRE].

[93] S.J. Gates, Jr., S.M. Kuzenko and A.G. Sibiryakov, $N=2$ supersymmetry of higher superspin massless theories, Phys. Lett. B 412 (1997) 59 [hep-th/9609141] [INSPIRE]. 
[94] M. Beccaria and A.A. Tseytlin, On induced action for conformal higher spins in curved background, Nucl. Phys. B 919 (2017) 359 [arXiv: 1702.00222] [INSPIRE].

[95] S.M. Kuzenko and G. Tartaglino-Mazzucchelli, Nilpotent chiral superfield in $N=2$ supergravity and partial rigid supersymmetry breaking, JHEP 03 (2016) 092 [arXiv: 1512.01964] [INSPIRE].

[96] S.M. Kuzenko, Maximally supersymmetric solutions of $R^{2}$ supergravity, Phys. Rev. D 94 (2016) 065014 [arXiv:1606.00654] [INSPIRE].

[97] C.R. Nappi and E. Witten, A WZW model based on a nonsemisimple group, Phys. Rev. Lett. 71 (1993) 3751 [hep-th/9310112] [INSPIRE].

[98] P. de Medeiros, J. Figueroa-O'Farrill and A. Santi, Killing superalgebras for Lorentzian four-manifolds, JHEP 06 (2016) 106 [arXiv:1605.00881] [INSPIRE].

[99] E.S. Fradkin and V.Y. Linetsky, Conformal superalgebras of higher spins, Mod. Phys. Lett. A 4 (1989) 2363.

[100] E.S. Fradkin and V. Ya. Linetsky, Conformal superalgebras of higher spins, Annals Phys. 198 (1990) 252 [INSPIRE].

[101] M.A. Vasiliev, Conformal higher spin symmetries of $4-D$ massless supermultiplets and osp (L,2M) invariant equations in generalized (super)space, Phys. Rev. D 66 (2002) 066006 [hep-th/0106149] [INSPIRE].

[102] M. Eastwood and T. Leistner, Higher symmetries of the square of the Laplacian, in: The IMA Volumes in Mathematics and its Applications. Vol. 144: Symmetries and Overdetermined Systems of Partial Differential Equations, M. Eastwood and W. Miller, Jr. eds., New York U.S.A. (2008), pg. 319.

[103] A.R. Gover and J. Šilhan, Higher symmetries of the conformal powers of the Laplacian on conformally flat manifolds, J. Math. Phys. 53 (2012) 032301 [arXiv:0911.5265].

[104] X. Bekaert and M. Grigoriev, Higher order singletons, partially massless fields and their boundary values in the ambient approach, Nucl. Phys. B 876 (2013) 667 [arXiv:1305.0162] [INSPIRE].

[105] T. Levasseur and J.T. Stafford, Higher symmetries of powers of the Laplacian and rings of differential operators, Compos. Math. 153 (2017) 678 [arXiv:1508.01664].

[106] X. Bekaert, S. Cnockaert, C. Iazeolla and M.A. Vasiliev, Nonlinear higher spin theories in various dimensions, in Higher spin gauge theories: Proceedings of 1st Solvay Workshop, Brussels Belgium (2004), pg. 132 [hep-th/0503128] [INSPIRE].

[107] M.A. Vasiliev, Multiparticle extension of the higher-spin algebra, Class. Quant. Grav. 30 (2013) 104006 [arXiv:1212.6071] [INSPIRE].

[108] O.A. Gelfond and M.A. Vasiliev, Operator algebra of free conformal currents via twistors, Nucl. Phys. B 876 (2013) 871 [arXiv:1301.3123] [INSPIRE].

[109] D. Butter and S.M. Kuzenko, Nonlocal action for the super-Weyl anomalies: A new representation, JHEP 09 (2013) 067 [arXiv:1307.1290] [INSPIRE].

[110] D. Butter, B. de Wit, S.M. Kuzenko and I. Lodato, New higher-derivative invariants in $N=2$ supergravity and the Gauss-Bonnet term, JHEP 12 (2013) 062 [arXiv:1307.6546] [INSPIRE]. 
[111] J. Hutomo and S.M. Kuzenko, Non-conformal higher spin supercurrents, Phys. Lett. B 778 (2018) 242 [arXiv: 1710.10837] [INSPIRE].

[112] J. Hutomo and S.M. Kuzenko, The massless integer superspin multiplets revisited, JHEP 02 (2018) 137 [arXiv: 1711.11364] [INSPIRE].

[113] I.L. Buchbinder, S.J. Gates and K. Koutrolikos, Higher Spin Superfield interactions with the Chiral Supermultiplet: Conserved Supercurrents and Cubic Vertices, Universe 4 (2018) 6 [arXiv: 1708.06262] [INSPIRE].

[114] K. Koutrolikos, P. Kočí and R. von Unge, Higher Spin Superfield interactions with Complex linear Supermultiplet: Conserved Supercurrents and Cubic Vertices, JHEP 03 (2018) 119 [arXiv: 1712.05150] [INSPIRE].

[115] I.L. Buchbinder, S.J. Gates and K. Koutrolikos, Conserved higher spin supercurrents for arbitrary spin massless supermultiplets and higher spin superfield cubic interactions, JHEP 08 (2018) 055 [arXiv: 1805.04413] [INSPIRE].

[116] L. Castellani, R. D'Auria and P. Fre, Supergravity and superstrings: A Geometric perspective. Vol. 2: Supergravity, World Scientific, Singapore (1991), pg. 680.

[117] M.F. Hasler, The Three form multiplet in $N=2$ superspace, Eur. Phys. J. C 1 (1998) 729 [hep-th/9606076] [INSPIRE].

[118] S.J. Gates, Jr., Ectoplasm has no topology: The Prelude, in Supersymmetries and Quantum Symmetries (SQS'97): Proceedings of 2nd International Seminar, dedicated to the Memory of V.I. Ogievetsky, Dubna Russia (1997), pg. 46 [hep-th/9709104] [INSPIRE].

[119] S.J. Gates, Jr., Ectoplasm has no topology, Nucl. Phys. B 541 (1999) 615 [hep-th/9809056] [INSPIRE].

[120] S.J. Gates, Jr., M.T. Grisaru, M.E. Knutt-Wehlau and W. Siegel, Component actions from curved superspace: Normal coordinates and ectoplasm, Phys. Lett. B 421 (1998) 203 [hep-th/9711151] [INSPIRE].

[121] P. Binetruy, F. Pillon, G. Girardi and R. Grimm, The Three form multiplet in supergravity, Nucl. Phys. B 477 (1996) 175 [hep-th/9603181] [INSPIRE].

[122] S.J. Gates, Jr., S.M. Kuzenko and G. Tartaglino-Mazzucchelli, Chiral supergravity actions and superforms, Phys. Rev. D 80 (2009) 125015 [arXiv: 0909.3918] [INSPIRE].

[123] S.M. Kuzenko and G. Tartaglino-Mazzucchelli, Complex three-form supergravity and membranes, JHEP 12 (2017) 005 [arXiv:1710.00535] [INSPIRE].

[124] D. Butter, S.M. Kuzenko and J. Novak, The linear multiplet and ectoplasm, JHEP 09 (2012) 131 [arXiv: 1205.6981] [INSPIRE]. 